\title{
The Mean Curvature of First-Order Submanifolds in Exceptional Geometries with Torsion
}

\author{
Gavin Ball and Jesse Madnick
}

September 2019

\begin{abstract}
We derive formulas for the mean curvature of associative 3 -folds, coassociative 4 -folds, and Cayley 4-folds in the general case where the ambient space has intrinsic torsion. Consequently, we are able to characterize those $\mathrm{G}_{2}$-structures (resp., $\operatorname{Spin}(7)$-structures) for which every associative 3 -fold (resp. coassociative 4 -fold, Cayley 4 -fold) is a minimal submanifold.

In the process, we obtain new obstructions to the local existence of coassociative 4 -folds in $\mathrm{G}_{2}$-structures with torsion.
\end{abstract}

\section{Contents}

1 Introduction $\quad \mathbf{2}$

1.1 Results on Associative 3-folds and Coassociative 4 -folds . . . . . . . . . . . . 3

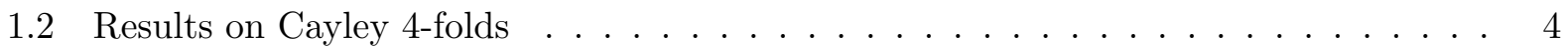

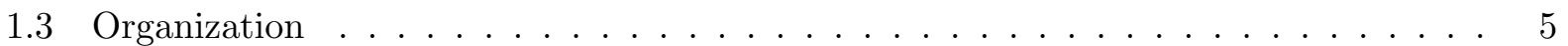

2 Associative 3-Folds and Coassociative 4-Folds in $\mathbf{G}_{2}$-Structures 5

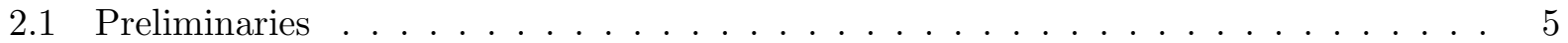

2.2 Some SO(4)-Representation Theory . . . . . . . . . . . . . . . . . 10

2.3 The Refined Torsion Forms . . . . . . . . . . . . . . . . . . . . . 15

2.4 Mean Curvature of Associative 3-Folds . . . . . . . . . . . . . . . . . . . 20

2.5 Mean Curvature of Coassociative 4 -Folds . . . . . . . . . . . . . . . . 22

3 Cayley 4-Folds in Spin(7)-Structures 25

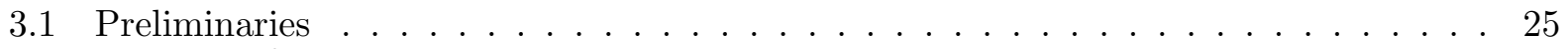

3.2 Some $\operatorname{Spin}^{h}(4)$-Representation Theory . . . . . . . . . . . . . . 27

3.3 The Refined Torsion Forms . . . . . . . . . . . . . . . . . . . 30

3.4 Mean Curvature of Cayley 4-Folds . . . . . . . . . . . . . . . . . . . . 34

$\begin{array}{ll}\text { References } & 37\end{array}$ 


\section{Introduction}

In their fundamental work on calibrations, Harvey and Lawson [9] defined four new classes of calibrated submanifolds in Riemannian manifolds with special holonomy, summarized in the following table:

\begin{tabular}{|l|l|}
\hline Submanifold & Ambient Manifold \\
\hline \hline Special Lagrangian $n$-fold & Riemannian 2n-manifold $\left(M^{2 n}, g\right)$ with $\operatorname{Hol}(g) \leq \mathrm{SU}(n)$ \\
\hline Associative 3-fold & Riemannian 7-manifold $\left(M^{7}, g\right)$ with $\operatorname{Hol}(g) \leq \mathrm{G}_{2}$ \\
\hline Coassociative 4-fold & Riemannian 7-manifold $\left(M^{7}, g\right)$ with $\operatorname{Hol}(g) \leq \mathrm{G}_{2}$ \\
\hline Cayley 4-fold & Riemannian 8-manifold $\left(M^{8}, g\right)$ with $\operatorname{Hol}(g) \leq \operatorname{Spin}(7)$ \\
\hline
\end{tabular}

By virtue of being calibrated, each of these submanifolds satisfy a strong area-minimizing property. In particular, they are stable minimal submanifolds. Moreover, by an argument using the CartanKähler Theorem, Harvey and Lawson [9] were able to show that submanifolds of each class exist locally in abundance.

Riemannian manifolds with special holonomy groups function as the background spaces for supersymmetric theories of physics. In this setting, calibrated submanifolds are related to supersymmetric cycles [3]. Special Lagrangian submanifolds lie at the foundation of the SYZ formulation of mirror symmetry [13], and calibrated submanifolds in manifolds with holonomy groups $\mathrm{G}_{2}$ and $\operatorname{Spin}(7)$ are expected to play a similar role in theories of mirror symmetry for such manifolds [1,8].

In fact, each of the classes of submanifolds described above make sense in an even more general class of ambient spaces: namely, that of (Riemannian) manifolds $M$ equipped with a $G$-structure, for $G=\mathrm{SU}(n)$ or $\mathrm{G}_{2}$ or $\operatorname{Spin}(7)$ as appropriate.

\begin{tabular}{|l|l|}
\hline Submanifold & Ambient Manifold \\
\hline \hline Special Lagrangian $n$-fold & 2n-manifold $M^{2 n}$ with an SU $(n)$-structure \\
\hline Associative 3-fold & 7-manifold $M^{7}$ with a $\mathrm{G}_{2}$-structure \\
\hline Coassociative 4-fold & 7-manifold $M^{7}$ with a $\mathrm{G}_{2}$-structure \\
\hline Cayley 4-fold & 8-manifold $M^{8}$ with a $\mathrm{Spin}(7)$-structure \\
\hline
\end{tabular}

However, in this generalized setting, such submanifolds need not be minimal. This raises the following:

Minimality Problem: Let $M$ be a manifold. Characterize those $G$-structures (for $G=\mathrm{SU}(n)$, $\mathrm{G}_{2}$, $\mathrm{Spin}(7)$ ) on $M$ for which every submanifold in $M$ of a given class (special Lagrangian, associative, etc.) is a minimal submanifold of $M$.

We will completely solve the Minimality Problem in the contexts of associative 3 -folds, and coassociative 4-folds, and Cayley 4-folds by deriving simple formulas for their mean curvature. The case of special Lagrangian 3-folds is addressed in our preprint [2].

Perhaps more fundamentally, in our generalized context the relevant submanifolds need not exist at all, even locally. This raises the natural:

Local Existence Problem: Let $M$ be a manifold. Characterize those $G$-structures (with $G$ as above) on $M$ for which submanifolds of a given class (special Lagrangian, etc.) exist locally at every point of $M$. 
In this work, we make progress towards the resolution of the Local Existence Problem in the setting of coassociatives. More precisely, we obtain an explicit obstruction to the local existence of coassociative 4-folds. Analogous obstructions to the local existence of special Lagrangian 3-folds are obtained in [2].

\subsection{Results on Associative 3-folds and Coassociative 4-folds}

Let $\left(M^{7}, \varphi\right)$ be a 7-manifold with a $\mathrm{G}_{2}$-structure $\varphi \in \Omega^{3}(M)$. The first-order local invariants of $\varphi$ are completely encoded in four differential forms, called the torsion forms of the $\mathrm{G}_{2}$-structure, denoted

$$
\left(\tau_{0}, \tau_{1}, \tau_{2}, \tau_{3}\right) \in \Omega^{0} \oplus \Omega^{1} \oplus \Omega^{2} \oplus \Omega^{3} .
$$

These are defined by the equations

$$
\begin{array}{rlrl}
d \varphi & =\tau_{0} * \varphi+3 \tau_{1} \wedge \varphi+* \tau_{3} \\
d * \varphi & = & 4 \tau_{1} \wedge * \varphi+\tau_{2} \wedge \varphi .
\end{array}
$$

together with the algebraic relations $*\left(\varphi \wedge \tau_{2}\right)=-\tau_{2}$ and $\tau_{3} \wedge \varphi=0$ and $\tau_{3} \wedge * \varphi=0$.

In order to study associative 3 -folds and coassociative 4 -folds in $M$, we will break the torsion forms into $\mathrm{SO}(4)$-irreducible pieces with respect to a certain splitting of $T M$. Indeed, in $\S 2.3$, we will decompose $\tau_{0}, \tau_{1}, \tau_{2}, \tau_{3}$ into $\mathrm{SO}(4)$-irreducible components, writing

$$
\begin{array}{ll}
\tau_{0}=\tau_{0} & \tau_{2}=\left(\tau_{2}\right)_{\mathbf{A}}+\left(\tau_{2}\right)_{1,3}+\left(\tau_{2}\right)_{2,0} \\
\tau_{1}=\left(\tau_{1}\right)_{\mathbf{A}}+\left(\tau_{1}\right)_{\mathbf{C}} & \tau_{3}=\left(\tau_{3}\right)_{0,0}+\left(\tau_{3}\right)_{0,4}+\left(\tau_{3}\right)_{2,2}+\left(\tau_{3}\right)_{1,3}+\left(\tau_{3}\right)_{\mathbf{C}}
\end{array}
$$

We will refer to the individual pieces

$$
\tau_{0}, \quad\left(\tau_{1}\right)_{\mathbf{A}},\left(\tau_{1}\right)_{\mathbf{C}}, \quad\left(\tau_{2}\right)_{\mathbf{A}},\left(\tau_{2}\right)_{1,3},\left(\tau_{2}\right)_{2,0}, \quad\left(\tau_{3}\right)_{0,0},\left(\tau_{3}\right)_{0,4},\left(\tau_{3}\right)_{2,2},\left(\tau_{3}\right)_{1,3},\left(\tau_{3}\right)_{\mathbf{C}}
$$

as refined torsion forms (with respect to a certain splitting of $T M$ ). The mean curvature of associatives and coassociatives can then be expressed purely in terms of the refined torsion. In the sequel, we let $\sharp: T^{*} M \rightarrow T M$ denote the usual musical (index-raising) isomorphism.

Theorem 2.18 (Mean Curvature of Associatives): The mean curvature vector $H$ of an associative 3 -fold in $M$ is given by

$$
H=-3\left[\left(\tau_{1}\right) \mathrm{C}\right]^{\sharp}-\frac{\sqrt{3}}{2}\left[\left(\tau_{3}\right)_{C}\right]^{\ddagger}
$$

where $\ddagger$ is a particular isometric isomorphism defined (2.18).

In particular, a $\mathrm{G}_{2}$-structure on $M$ has the property that every associative 3 -fold in $M$ is minimal if and only if $\tau_{1}=\tau_{3}=0$. Equivalently, if and only if $d \varphi=\lambda * \varphi$ for some constant $\lambda \in \mathbb{R}$.

Theorem 2.21 (Mean Curvature of Coassociatives): The mean curvature vector $H$ of a coassociative 4 -fold in $M$ is given by

$$
H=-4\left[\left(\tau_{1}\right)_{\mathrm{A}}\right]^{\sharp}+\frac{\sqrt{6}}{3}\left[\left(\tau_{2}\right)_{\mathrm{A}}\right]^{\natural}
$$

where $\measuredangle$ is a particular isometric isomorphism defined in (2.11).

In particular, a $\mathrm{G}_{2}$-structure on $M$ has the property that every coassociative 4 -fold in $M$ is minimal if and only if $\tau_{1}=\tau_{2}=0$. Equivalently, if and only if $d * \varphi=0$. 
These formulas can be regarded as a submanifold analogue of the curvature formulas derived by Bryant [5] for 7-manifolds with $\mathrm{G}_{2}$-structures. In the process of proving Theorem 2.21, we will observe an obstruction to the local existence of coassociative 4-folds:

Theorem 2.19 (Local Obstruction to Coassociatives): If a coassociative 4 -fold $\Sigma$ exists in $M$, then the following relation holds at points of $\Sigma$ :

$$
\tau_{0}=-\frac{\sqrt{42}}{7}\left[\left(\tau_{3}\right)_{0,0}\right]^{\dagger}
$$

where $\dagger$ is an isometric isomorphism defined in (2.17).

In particular, if $\tau_{3}=0$ and $\tau_{0}$ is non-vanishing, then $M$ admits no coassociative 4 -folds (even locally).

Corollary 2.20: Fix $x \in M$. If every coassociative 4-plane in $T_{x} M$ is tangent to a coassociative 4-fold, then $\left.\tau_{0}\right|_{x}=0$ and $\left.\tau_{3}\right|_{x}=0$.

Note that Theorem 2.19 generalizes the well-known fact that nearly-parallel $\mathrm{G}_{2}$-structures (viz., those with $\tau_{1}=\tau_{2}=\tau_{3}=0$ and $\tau_{0}$ non-vanishing) admit no coassociative 4 -folds.

\subsection{Results on Cayley 4-folds}

Let $\left(M^{8}, \Phi\right)$ be an 8-manifold with a $\operatorname{Spin}(7)$-structure $\Phi \in \Omega^{4}(M)$. The first-order local invariants of $\Phi$ are completely encoded in two differential forms, $\tau_{1} \in \Omega^{1}(M)$ and $\tau_{3} \in \Omega^{3}(M)$, called the torsion forms of the Spin(7)-structure. They are defined by equation

$$
d \Phi=\tau_{1} \wedge \Phi+* \tau_{3} .
$$

To study Cayley 4 -folds in $M$, we will break the torsion forms into $\operatorname{Spin}^{h}(4)$-irreducible pieces with respect to a certain splitting of $T M$, where here $\operatorname{Spin}^{h}(4)=(\mathrm{SU}(2) \times \mathrm{SU}(2) \times \mathrm{SU}(2)) / \mathbb{Z}_{2}$ is the stabilizer of a Cayley 4-plane. Indeed, in $\S 3.3$, we will decompose $\tau_{1}$ and $\tau_{3}$ into irreducible pieces, writing

$$
\begin{aligned}
& \tau_{1}=\left(\tau_{1}\right)_{\mathbf{K}}+\left(\tau_{1}\right)_{\mathbf{L}} \\
& \tau_{3}=\left(\tau_{3}\right)_{\mathbf{K}}+\left(\tau_{3}\right)_{\mathbf{L}}+\left(\tau_{3}\right)_{0,3,1}+\left(\tau_{3}\right)_{2,1,1}+\left(\tau_{3}\right)_{1,3,0}+\left(\tau_{3}\right)_{1,1,2}
\end{aligned}
$$

and refer to the individual pieces

$$
\left(\tau_{1}\right)_{\mathbf{K}},\left(\tau_{1}\right)_{\mathbf{L}}, \quad\left(\tau_{3}\right)_{\mathbf{K}},\left(\tau_{3}\right)_{\mathbf{L}},\left(\tau_{3}\right)_{0,3,1},\left(\tau_{3}\right)_{2,1,1},\left(\tau_{3}\right)_{1,3,0}
$$

as refined torsion forms (with respect to a certain splitting of $T M$ ). As with associative and coassociative submanifolds, the mean curvature of Cayley submanifolds can be expressed purely in terms of the refined torsion. As before, we let $\sharp: T^{*} M \rightarrow T M$ denote the musical isomorphism.

Theorem 3.12 (Mean Curvature of Cayleys): The mean curvature vector $H$ of a Cayley 4 -fold in $M$ is given by

$$
H=-\left[\left(\tau_{1}\right)_{\mathrm{L}}\right]^{\sharp}-\frac{\sqrt{42}}{7}\left[\left(\tau_{3}\right)_{\mathrm{L}}\right]^{\dagger}
$$

where $\nmid$ is a particular isometric isomorphism defined in Definition 3.11.

In particular, a Spin(7)-structure on $M$ has the property that every Cayley 4 -fold in $M$ is minimal if and only if $d \Phi=0$. 


\subsection{Organization}

In $\S 2$, we study associative 3 -folds and coassociative 4-folds in 7 -manifolds with $\mathrm{G}_{2}$-structures. We use $\S 2.1$ to recall basic aspects of $\mathrm{G}_{2}$ geometry and set notation. In $\S 2.2$, we will decompose various $\mathrm{G}_{2}$-modules (viz., $\Lambda^{k}\left(\mathbb{R}^{7}\right)$ and $\operatorname{Sym}^{2}\left(\mathbb{R}^{7}\right)$ ) into $\mathrm{SO}(4)$-irreducible pieces, where we think of $\mathrm{SO}(4)$ as the stabilizer of an associative (or coassociative) plane. These $\mathrm{SO}(4)$-submodules are central to the geometry of associative and coassociative submanifolds, and we will take care to provide explicit descriptions of these submodules as much as possible.

In $\S 2.3$, we will define the refined torsion forms by way of the $\mathrm{SO}(4)$-irreducible decompositions obtained in $\S 2.2$. Once these refined torsion forms are defined, we will express them in terms of a local $\mathrm{SO}(4)$-frame in order to perform calculations.

Finally, in $\S 2.4$ we will apply this machinery to the study of associative 3 -folds, proving Theorem 2.18. Similarly, in $\S 2.5$, we turn to coassociative 4-folds, proving Theorem 2.19, Corollary 2.20, and Theorem 2.21.

The structure of $\S 3$ is completely analogous. In brief, we use $\S 3.1$ to recall the basic aspects of $\operatorname{Spin}(7)$ geometry and set notation. In $\S 3.2$, we decompose various $\operatorname{Spin}(7)$-modules into $\operatorname{Spin}^{h}(4)$ representations, where we continue to write $\operatorname{Spin}^{h}(4)=(\mathrm{SU}(2) \times \mathrm{SU}(2) \times \mathrm{SU}(2)) / \mathbb{Z}_{2}$, regarded as the stabilizer of a Cayley 4-plane. In $\S 3.3$, we will define the corresponding refined torsion forms by way of $\operatorname{Spin}^{h}(4)$-representation theory, and then express them in terms of a local $\operatorname{Spin}^{h}(4)$-frame. Finally, in $§ 3.4$, we will study Cayley 4-folds and prove Theorem 3.12.

Acknowledgements: This work has benefited from conversations with Robert Bryant, Jason Lotay, Thomas Madsen, and Alberto Raffero. The second author would also like to thank McKenzie Wang for his guidance and encouragement. The first author thanks the Simons Collaboration on Special Holonomy in Geometry, Analysis and Physics for support during the period in which this article was written.

After this work was completed, it was pointed out to us that the mean curvature formula for associative 3-folds, Theorem 2.18, was derived earlier by Paul Reynolds in his $2011 \mathrm{PhD}$ thesis [10]. In that work, Reynolds also derived an expression for the mean curvature of Cayley 4-folds, but his expression is not completely split into irreducible pieces.

\section{Associative 3-Folds and Coassociative 4-Folds in $\mathbf{G}_{2}$-Structures}

Our goal in this section is to derive formulas (Theorems 2.18 and 2.21) for the mean curvature of associative 3 -folds and coassociative 4 -folds in 7 -manifolds equipped with a $\mathrm{G}_{2}$-structure. We will also derive an obstruction (Theorem 2.19) to the local existence of coassociative 4-folds.

These formulas and obstructions will be phrased in terms of refined torsion forms, which we will define in $\S 2.3$. These refined forms are essentially the $\mathrm{SO}(4)$-irreducible pieces of the usual torsion forms $\tau_{0}, \tau_{1}, \tau_{2}, \tau_{3}$ of a $\mathrm{G}_{2}$-structure. As such, we will devote $\S 2.2$ to the relevant $\mathrm{SO}(4)$ representation theory needed to decompose $\tau_{0}, \tau_{1}, \tau_{2}, \tau_{3}$.

\subsection{Preliminaries}

In this subsection, we define both the ambient spaces (in $\S 2.1 .2$ ) and submanifolds (in $\S 2.1 .3$ ) of interest. We also use this subsection to fix notation and clarify conventions. 


\subsection{1 $\mathrm{G}_{2}$-Structures on Vector Spaces}

Let $V=\mathbb{R}^{7}$ equipped with the standard inner product $\langle\cdot, \cdot\rangle$, norm $\|\cdot\|$, and an orientation. Let $\left\{e_{1}, \ldots, e_{7}\right\}$ denote the standard (orthonormal) basis of $V$, and let $\left\{e^{1}, \ldots, e^{7}\right\}$ denote the corresponding dual basis of $V^{*}$. The associative 3 -form is the alternating 3 -form $\phi_{0} \in \Lambda^{3}\left(V^{*}\right)$ defined by

$$
\phi_{0}=e^{123}+e^{1} \wedge\left(e^{45}+e^{67}\right)+e^{2} \wedge\left(e^{46}-e^{57}\right)+e^{3} \wedge\left(-e^{47}-e^{56}\right)
$$

The coassociative 4-form is the alternating 4-form $* \phi_{0} \in \Lambda^{4}\left(V^{*}\right)$ given by the Hodge dual $*$ of $\phi_{0}$. Explicitly:

$$
* \phi_{0}=e^{4567}+e^{23} \wedge\left(e^{45}+e^{67}\right)+e^{13} \wedge\left(-e^{46}+e^{57}\right)+e^{12} \wedge\left(-e^{47}-e^{56}\right) .
$$

For calculations, it will be convenient to express $\phi_{0}$ and $* \phi_{0}$ in the form

$$
\phi_{0}=\frac{1}{6} \epsilon_{i j k} e^{i j k} \quad * \phi_{0}=\frac{1}{24} \epsilon_{i j k \ell} e^{i j k \ell}
$$

where the constants $\epsilon_{i j k}, \epsilon_{i j k \ell} \in\{-1,0,1\}$ are defined by this formula. For example, $\epsilon_{123}=\epsilon_{145}=1$ and $\epsilon_{347}=\epsilon_{356}=-1$. Identities involving the $\epsilon$-symbols are given in [5].

Remark. The associative and coassociative forms admit simple descriptions via the algebra of the octonions $\mathbb{O}$.

Equip $\mathbb{O} \simeq \mathbb{R}^{8}$ with the standard (euclidean) inner product and split $\mathbb{O}=\operatorname{Re}(\mathbb{O}) \oplus \operatorname{Im}(\mathbb{O}) \simeq \mathbb{R} \oplus$ $\mathbb{R}^{7}$, where $\operatorname{Re}(\mathbb{O}):=\operatorname{span}_{\mathbb{R}}(1)$ is the real line and $\operatorname{Im}(\mathbb{O}):=\operatorname{Re}(\mathbb{O})^{\perp}$ is its orthogonal complement. Under the identification $V \simeq \operatorname{Im}(\mathbb{O})$, the associative and coassociative forms are given by

$$
\begin{aligned}
\phi_{0}(x, y, z) & =\langle x, y \times z\rangle \\
* \phi_{0}(x, y, z, w) & =\frac{1}{2}\langle x,[y, z, w]\rangle,
\end{aligned}
$$

for $x, y, z \in V$, where $y \times z:=\operatorname{Im}(\bar{z} y)=\frac{1}{2}(\bar{z} y-\bar{y} z)$ is the octonionic cross product, and $[y, z, w]:=$ $(y z) w-y(z w)$ is the associator, measuring the failure of associativity of multiplication in $\operatorname{Im}(\mathbb{O})$. See [9] for a proof.

Consider the GL(V)-action on $\Lambda^{3}\left(V^{*}\right)$ given by pullback: $A \cdot \gamma:=A^{*} \gamma$ for $A \in \mathrm{GL}(V)$ and $\gamma \in \Lambda^{3}\left(V^{*}\right)$. It is a classical result of Schouten (see [Bryant 87] for a proof) that the stabilizer of $\phi_{0} \in \Lambda^{3}\left(V^{*}\right)$ is the compact Lie group $\mathrm{G}_{2}$, i.e.:

$$
\mathrm{G}_{2} \cong\left\{A \in \mathrm{GL}(V): A^{*} \phi_{0}=\phi_{0}\right\}
$$

We let $\Lambda_{+}^{3}\left(V^{*}\right)$ denote the orbit of $\phi_{0} \in \Lambda^{3}\left(V^{*}\right)$ under this $\mathrm{G}_{2}$-action, i.e.:

$$
\Lambda_{+}^{3}\left(V^{*}\right):=\left\{A^{*} \phi_{0}: A \in \mathrm{GL}(V)\right\} \cong \frac{\mathrm{GL}(V)}{\mathrm{G}_{2}} .
$$

In [5], it is noted that $\Lambda_{+}^{3}\left(V^{*}\right) \subset \Lambda^{3}\left(V^{*}\right) \simeq \mathbb{R}^{35}$ is an open subset with two connected components, each diffeomorphic to $\mathbb{R} \mathbb{P}^{7} \times \mathbb{R}^{28}$.

The isomorphism $\mathrm{G}_{2} \cong\left\{A \in \mathrm{GL}(V): A^{*} \phi_{0}=\phi_{0}\right\}$ lets us regard $\mathrm{G}_{2}$ as a subgroup of $\mathrm{GL}(V)$, which in turn lets us view $V \simeq \mathbb{R}^{7}$ as a faithful $\mathrm{G}_{2}$-representation. It can be shown (see [Bryant 87]) that this $\mathrm{G}_{2}$-representation is irreducible.

However, the induced $\mathrm{G}_{2}$-representations on $\Lambda^{k}\left(V^{*}\right)$ for $2 \leq k \leq 5$ are not irreducible. Indeed, $\Lambda^{2}\left(V^{*}\right)$ decomposes into irreducible $\mathrm{G}_{2}$-modules as

$$
\Lambda^{2}\left(V^{*}\right)=\Lambda_{7}^{2} \oplus \Lambda_{14}^{2}
$$


where

$$
\begin{gathered}
\Lambda_{7}^{2}=\left\{\beta \in \Lambda^{2}\left(V^{*}\right): *\left(\phi_{0} \wedge \beta\right)=2 \beta\right\} \\
\Lambda_{14}^{2}=\left\{\beta \in \Lambda^{2}\left(V^{*}\right): *\left(\phi_{0} \wedge \beta\right)=-\beta\right\}
\end{gathered}
$$

Similarly, $\Lambda^{3}\left(V^{*}\right)$ decomposes into irreducible $\mathrm{G}_{2}$-modules as

$$
\Lambda^{3}\left(V^{*}\right)=\Lambda_{1}^{3} \oplus \Lambda_{7}^{3} \oplus \Lambda_{27}^{3}
$$

where

$$
\begin{aligned}
\Lambda_{1}^{3} & =\mathbb{R} \phi_{0} \\
\Lambda_{7}^{3} & =\left\{*\left(\alpha \wedge \phi_{0}\right): \alpha \in \Lambda^{1}\right\} \\
\Lambda_{27}^{3} & =\left\{\gamma \in \Lambda^{3}: \gamma \wedge \phi_{0}=0 \text { and } \gamma \wedge * \phi_{0}=0\right\} .
\end{aligned}
$$

In each case, $\Lambda_{\ell}^{k}$ is an irreducible $\mathrm{G}_{2}$-module of dimension $\ell$. Via the Hodge star $*: \Lambda^{k}\left(V^{*}\right) \rightarrow$ $\Lambda^{7-k}\left(V^{*}\right)$, one can obtain similar decompositions of $\Lambda^{4}\left(V^{*}\right)$ and $\Lambda^{5}\left(V^{*}\right)$.

In the sequel, we will always equip $\Lambda^{k}\left(V^{*}\right)$ with the usual inner product, also denoted $\langle\cdot, \cdot\rangle$, given by declaring

$$
\left\{e^{I}: I \text { increasing multi-index }\right\}
$$

to be an orthonormal basis. We let $\|\cdot\|$ denote the corresponding norm.

For our calculations in $\S 2.2$, we will need the $\mathrm{G}_{2}$-equivariant map $\mathrm{i}$, defined on decomposable elements of $\operatorname{Sym}_{0}^{2}\left(V^{*}\right)$ as follows:

$$
\begin{aligned}
\mathrm{i}: \operatorname{Sym}_{0}^{2}\left(V^{*}\right) & \rightarrow \Lambda^{3}\left(V^{*}\right) \\
\mathrm{i}(\alpha \circ \beta) & =\alpha \wedge *\left(\beta \wedge * \phi_{0}\right)+\beta \wedge *\left(\alpha \wedge * \phi_{0}\right) .
\end{aligned}
$$

It is shown in [5] that the image of $\mathrm{i}$ is $\Lambda_{27}^{3}$, so that the map with restricted image $\mathrm{i}: \operatorname{Sym}_{0}^{2}\left(V^{*}\right) \rightarrow \Lambda_{27}^{3}$ is an isomorphism of $\mathrm{G}_{2}$-modules. It is also remarked that with respect to the orthonormal basis $\left\{e^{1}, \ldots, e^{7}\right\}$ of $V^{*}$, one has

$$
\mathrm{i}\left(h_{i j} e^{i} \circ e^{j}\right)=\epsilon_{i k \ell} h_{i j} e^{j k \ell} .
$$

To invert i, one can use the map

$$
\begin{aligned}
\mathrm{j}: \Lambda_{27}^{3}\left(V^{*}\right) & \rightarrow \operatorname{Sym}_{0}^{2}\left(V^{*}\right) \\
\mathrm{j}(\gamma)(v, w) & =*\left(\iota_{v} \phi_{0} \wedge \iota_{w} \phi_{0} \wedge \gamma\right)
\end{aligned}
$$

which satisfies $\mathrm{j} \circ \mathrm{i}=8 \mathrm{Id}_{\mathrm{Sym}_{0}^{2}\left(V^{*}\right)}$.

Finally, we remark that from the associative 3 -form $\phi_{0}$, one can recover the inner product $\langle\cdot, \cdot\rangle$ and volume form vol $=e^{1 \cdots 7}$ via

$$
\begin{aligned}
\langle X, Y\rangle \operatorname{vol} & =\frac{1}{6}\left(\iota_{X} \phi_{0}\right) \wedge\left(\iota_{Y} \phi_{0}\right) \wedge \phi_{0} \\
\operatorname{vol} & =\phi_{0} \wedge * \phi_{0} .
\end{aligned}
$$

From these identities, one can show that, in fact, $\mathrm{G}_{2}$ preserves both $\langle\cdot, \cdot\rangle$ and the orientation on $V$, so we may regard $\mathrm{G}_{2} \leq \mathrm{SO}(V,\langle\cdot, \cdot\rangle) \simeq \mathrm{SO}(7)$. 


\subsection{2 $\mathrm{G}_{2}$-Structures on 7-Manifolds}

Definition 2.1. Let $M$ be an oriented 7-manifold. A $G_{2}$-structure on $M$ is a differential 3-form $\varphi \in \Omega^{3}(M)$ such that $\left.\varphi\right|_{x} \in \Lambda_{+}^{3}\left(T_{x}^{*} M\right)$ at each $x \in M$. That is, at each $x \in M$, there exists a coframe $u: T_{x} M \rightarrow \mathbb{R}^{7}$ for which $\left.\varphi\right|_{x}=u^{*}\left(\phi_{0}\right)$.

Intuitively, a $\mathrm{G}_{2}$-structure is a smooth identification of each tangent space $T_{x} M$ with $\operatorname{Im}(\mathbb{O})$ in such a way that $\left.\varphi\right|_{x}$ and $\phi_{0}$ are aligned: $\left(T_{x} M,\left.\varphi\right|_{x}\right) \simeq\left(\operatorname{Im}(\mathbb{O}), \phi_{0}\right)$. We remark that a 7-manifold $M$ admits a $\mathrm{G}_{2}$-structure if and only if it is orientable and spin: see [5] for a proof.

Every $\mathrm{G}_{2}$-structure $\varphi$ on $M$ induces a Riemannian metric $g_{\varphi}$ and an orientation form $\operatorname{vol}_{\varphi}$ on $M$ via the formulas (2.3), reflecting the inclusion $\mathrm{G}_{2} \leq \mathrm{SO}(7)$. We caution, however, that the association $\varphi \mapsto g_{\varphi}$ is not injective: different $\mathrm{G}_{2}$-structures may induce the same Riemannian metric. For a discussion of this point, see [5].

The first-order local invariants of a $\mathrm{G}_{2}$-structure are completely encoded in a certain $\mathrm{G}_{2^{-}}$ equivariant function

$$
T: F_{\mathrm{G}_{2}} \rightarrow \Lambda^{0} \oplus \Lambda^{1} \oplus \Lambda_{14}^{2} \oplus \Lambda_{27}^{3} \simeq \mathbb{R}^{49}
$$

called the intrinsic torsion function, defined on the total space of the $\mathrm{G}_{2}$-frame bundle $F_{\mathrm{G}_{2}} \rightarrow M$ over $M$. We think of $T$ as describing the 1 -jet of the $\mathrm{G}_{2}$-structure.

The intrinsic torsion function is somewhat technical to define - the interested reader can find more information in [7] and [12] — but several equivalent reformulations are available. Most conveniently for our purposes: the intrinsic torsion function of a $\mathrm{G}_{2}$-structure is equivalent to the data of the 4-form $d \varphi$ and the 5 -form $d * \varphi$. In [5], the exterior derivatives of $\varphi$ and $* \varphi$ are shown to take the form

$$
\begin{array}{rlrl}
d \varphi & =\tau_{0} * \varphi+3 \tau_{1} \wedge \varphi+* \tau_{3} \\
d * \varphi & = & 4 \tau_{1} \wedge * \varphi+\tau_{2} \wedge \varphi .
\end{array}
$$

where

$$
\left(\tau_{0}, \tau_{1}, \tau_{2}, \tau_{3}\right) \in \Gamma\left(\Lambda^{0}\left(T^{*} M\right) \oplus \Lambda^{1}\left(T^{*} M\right) \oplus \Lambda_{14}^{2}\left(T^{*} M\right) \oplus \Lambda_{27}^{3}\left(T^{*} M\right)\right)
$$

We refer to $\tau_{0}, \tau_{1}, \tau_{2}, \tau_{3}$ as the torsion forms of the $\mathrm{G}_{2}$-structure.

Following standard conventions, we let $W_{1}, W_{7}, W_{14}, W_{27}$ denote the vector bundles $\Lambda^{0}\left(T^{*} M\right)$, $\Lambda^{1}\left(T^{*} M\right), \Lambda_{14}^{2}\left(T^{*} M\right), \Lambda_{27}^{3}\left(T^{*} M\right)$, respectively. Consider the set $\mathcal{S}$ consisting of the $2^{4}=16$ vector bundles

$$
\mathcal{S}=\left\{0, W_{i}, W_{i} \oplus W_{j}, W_{i} \oplus W_{j} \oplus W_{k}, W_{1} \oplus W_{7} \oplus W_{14} \oplus W_{27}: i, j, k \in\{1,7,14,27\}\right\} .
$$

Definition 2.2. Let $E \in \mathcal{S}$ be a vector bundle on the list above. We say that a $\mathrm{G}_{2}$-structure belongs to the torsion class $E$ if and only if the torsion forms of the $\mathrm{G}_{2}$-structure $\left(\tau_{0}, \tau_{1}, \tau_{2}, \tau_{3}\right) \in$ $\Gamma\left(W_{1} \oplus W_{7} \oplus W_{14} \oplus W_{27}\right)$ is valued in $E \subset W_{1} \oplus W_{7} \oplus W_{14} \oplus W_{27}$.

For example, a $\mathrm{G}_{2}$-structure belongs to the torsion class $W_{7} \oplus W_{27}$ if and only if $\tau_{0}=\tau_{2}=0$.

\subsubsection{Associative 3-Folds and Coassociative 4-Folds}

Let $\left(M^{7}, \varphi\right)$ be a 7 -manifold with a $\mathrm{G}_{2}$-structure, and consider a tangent space $\left(T_{x} M,\left.\varphi\right|_{x}\right) \simeq$ $\left(V, \phi_{0}\right)$. The vector space $\left(V, \phi_{0}\right)$ possesses two distinguished classes of subspaces - associative 3planes and coassociative 4-planes (to be defined shortly) — first studied by Harvey and Lawson [9]

in their work on calibrations. Indeed, they observed that $\phi_{0}$ and $* \phi_{0}$ enjoy the following remarkable property: 
Proposition $2.3([9])$. The associative 3 -form $\phi_{0}$ and coassociative 4 -form $* \phi_{0}$ have co-mass one, meaning that

$$
\left|\phi_{0}(x, y, z)\right| \leq 1 \quad\left|* \phi_{0}(x, y, z, w)\right| \leq 1
$$

for every orthonormal set $\{x, y, z, w\}$ in $V \simeq \mathbb{R}^{7}$.

In light of this proposition, it is natural to examine more closely those 3-planes $A \in \mathrm{Gr}_{3}(V)$ (respectively, 4-planes $C \in \mathrm{Gr}_{4}(V)$ ) for which $\left|\phi_{0}(A)\right|=1$ (resp., $\left|* \phi_{0}(C)\right|=1$ ).

Proposition $2.4([9])$. Let $A \in G r_{3}(V)$ be a 3-plane in $V$. The following are equivalent:

1. If $\{u, v, w\}$ orthonormal basis of $A$, then $\phi_{0}(u, v, w)= \pm 1$.

2. For all $u, v, w \in A$, we have $[u, v, w]=0$.

3. $A=\operatorname{span}\{u, v, u \times v\}$ for some linearly independent set $\{u, v\}$.

If any of these conditions hold, we say that $A$ is an associative 3-plane.

Proposition $2.5([9])$. Let $C \in G r_{4}(V)$ be a 4-plane in $V$. The following are equivalent:

1. If $\{x, y, z, w\}$ is an orthonormal basis of $C$, then $* \phi_{0}(x, y, z, w)= \pm 1$.

2. $C^{\perp}$ is associative.

3. $\left.\phi_{0}\right|_{C}=0$.

If any of these conditions hold, we say that $C$ is a coassociative 4-plane.

Proofs of the above propositions can be found in [9] and [11].

The $\mathrm{G}_{2}$-action on $V$ induces $\mathrm{G}_{2}$-actions on the Grassmannians $\operatorname{Gr}_{k}(V)$ of $k$-planes in $V$. While these actions are transitive for $k=1,2,5,6$, they are not transitive for $k=3,4$ : indeed, the (proper) subsets consisting of associative 3-planes and coassociative 4-planes are $\mathrm{G}_{2}$-orbits. The corresponding stabilizer, recorded in the following proposition, will play a crucial role in this work:

Proposition 2.6 ([9]). The Lie group $G_{2}$ acts transitively on the subset of associative 3-planes and on the subset of coassociative 4-planes:

$$
\begin{aligned}
\left\{E \in G r_{3}(V):\left|\phi_{0}(E)\right|\right. & =1\} \subset G r_{3}(V), \\
\left\{E \in G r_{4}(V):\left|* \phi_{0}(E)\right|\right. & =1\} \subset G r_{4}(V) .
\end{aligned}
$$

In both cases, the stabilizer of the $\mathrm{G}_{2}$-action is isomorphic to $\mathrm{SO}(4)$.

We may finally define our primary objects of interest:

Definition 2.7. Let $\left(M^{7}, \varphi\right)$ be a 7 -manifold equipped with a $\mathrm{G}_{2}$-structure $\varphi$. Identify each tangent space $\left(T_{x} M,\left.\varphi\right|_{x}\right) \simeq\left(V, \phi_{0}\right)$.

An associative 3-fold in $M$ is a 3-dimensional immersed submanifold $\Sigma \subset M$ for which each tangent space $T_{x} \Sigma \subset T_{x} M$ is an associative 3-plane.

Similarly, a coassociative 4-fold in $M$ is a 4-dimensional immersed submanifold $\Sigma \subset M$ for which each tangent space $T_{x} \Sigma \subset T_{x} M$ is a coassociative 4-plane.

Note that if $d \varphi=0$, then $\varphi$ is a calibration whose calibrated 3-planes are the associative 3planes in $T_{x} M$. Thus, in this case, an associative 3 -fold is a calibrated submanifold, and hence a minimal submanifold of $M$.

Similarly, if $d * \varphi=0$, then $* \varphi$ is a calibration whose calibrated 4-planes are the coassociative 4-planes in $T_{x} M$. Thus, in this case, a coassociative 4 -fold is a calibrated submanifold, and hence a minimal submanifold of $M$. The "Minimality Problem" described in the introduction asks about the converses of these claims. 


\subsection{Some SO(4)-Representation Theory}

In this subsection, we describe the aspects of $\mathrm{SO}(4)$-representation theory that are relevant to the study of associative 3-folds and coassociative 4-folds. Particularly important for our purposes are $\mathrm{SO}(4)$-irreducible decompositions of $\Lambda^{1}\left(\mathbb{R}^{7}\right), \Lambda^{2}\left(\mathbb{R}^{7}\right)$, $\operatorname{Sym}^{2}\left(\mathbb{R}^{7}\right)$, and $\Lambda^{3}\left(\mathbb{R}^{7}\right)$, each of which we will describe in turn.

To begin, recall that the compact Lie group $\mathrm{SO}(4)$ is double-covered by the simply-connected group $\mathrm{SU}(2) \times \mathrm{SU}(2)$. The complex irreducible representations of $\mathrm{SU}(2) \times \mathrm{SU}(2)$ are exactly the tensor products $\mathrm{V}_{p} \otimes \mathrm{V}_{q}$ of irreducible $\mathrm{SU}(2)$-representations for each factor. The complex irreducible representations of $\mathrm{SU}(2)$ are well known to be the spaces of homogeneous polynomials in two variables of fixed degree, $\mathrm{V}_{p}=\operatorname{Sym}^{p}(\mathbb{C}\langle x, y\rangle)$.

Let $\mathrm{V}_{p, q}^{\mathbb{C}}$ denote $\mathrm{V}_{p} \otimes \mathrm{V}_{q}$. We think of $\mathrm{V}_{p, q}^{\mathbb{C}}$ as the space of homogeneous polynomials in $(x, y ; w, z)$ of bidegree $(p, q)$. When $p$ and $q$ have the same parity the representation $\bigvee_{p, q}^{\mathbb{C}}$ descends to a representation of $\mathrm{SO}(4)$, and each of these representations has a real structure induced by the map $(x, y, w, z) \mapsto(y,-x, z,-w)$. This yields a complete description of the real representations of $\mathrm{SO}(4)$.

We work with real representations, letting $\mathrm{V}_{p, q}$ denote the real representation underlying $\mathrm{V}_{p, q}^{\mathbb{C}}$. In this language, the standard 4-dimensional representation of $\mathrm{SO}(4)$ is $\mathrm{V}_{1,1}$, while the adjoint representation $\mathfrak{s o}(4)$ is $\mathrm{V}_{2,0} \oplus \mathrm{V}_{0,2}$. The ordering of the subscripts is chosen so that the representation $\Lambda_{+}^{2}\left(\mathbb{R}^{4}\right)$ of $\mathrm{SO}(4)$ on the self-dual 2 -forms is $\mathrm{V}_{0,2}$.

The Clebsch-Gordan formula applied to each $\mathrm{SU}(2)$ representation gives the irreducible decomposition of a tensor product of $\mathrm{SO}(4)$-modules:

$$
\mathrm{V}_{p_{1}, q_{1}} \otimes \mathrm{V}_{p_{2}, q_{2}} \cong \bigoplus_{i=0}^{\left|p_{1}-p_{2}\right|\left|q_{1}-q_{2}\right|} \bigoplus_{j=0} \mathrm{~V}_{p_{1}+p_{2}-2 i, q_{1}+q_{2}-2 j}
$$

\subsection{1 $\mathrm{SO}(4)$ as a subgroup of $\mathrm{G}_{2}$}

In our calculations we shall need a concrete realization of $\mathrm{SO}(4)$ as the stabilizer of an associative or coassociative plane. Let $\mathrm{SO}(4)$ act on $V \cong \mathbb{R}^{7}$ via the identification $V \cong \mathrm{V}_{0,2} \oplus \mathrm{V}_{1,1}$, and let $\left(e_{1}, \ldots, e_{7}\right)$ be an orthonormal basis of $V$ such that:

- $\left\langle e_{1}, e_{2}, e_{3}\right\rangle \cong \mathrm{V}_{0,2}$ and $\left\langle e_{4}, e_{5}, e_{6}, e_{7}\right\rangle \cong \mathrm{V}_{1,1}$,

- The map

$$
e_{1} \mapsto e_{45}+e_{67}, \quad e_{2} \mapsto e_{46}-e_{57}, \quad e_{3} \mapsto-e_{47}-e_{56}
$$

is $\mathrm{SO}(4)$-equivariant.

Then the 3-form

$$
e^{123}+e^{1} \wedge\left(e^{45}+e^{67}\right)+e^{2} \wedge\left(e^{46}-e^{57}\right)+e^{3} \wedge\left(-e^{47}-e^{56}\right)
$$

is $\mathrm{SO}(4)$-invariant, and thus the action of $\mathrm{SO}(4)$ on $V$ gives an embedding $\mathrm{SO}(4) \subset \mathrm{G}_{2}$. The 3-plane $\left\langle e_{1}, e_{2}, e_{3}\right\rangle$ is associative and preserved by the action of $\mathrm{SO}(4)$, while the 4 -plane $\left\langle e_{4}, e_{5}, e_{6}, e_{7}\right\rangle$ is coassociative and preserved by the action of $\mathrm{SO}(4)$.

\subsubsection{Decomposition of 1-Forms on $V^{*}$}

Let $V$ be as above. The decomposition of $\Lambda^{1}\left(V^{*}\right)$ into irreducible $\mathrm{SO}(4)$-modules is simply

$$
\Lambda^{1}\left(V^{*}\right)=\mathrm{A} \oplus \mathrm{C}
$$


where

$$
\mathrm{A} \cong\left\langle e^{1}, e^{2}, e^{3}\right\rangle, \quad \mathrm{C} \cong\left\langle e^{4}, e^{5}, e^{6}, e^{7}\right\rangle .
$$

As abstract $\mathrm{SO}(4)$-modules, we have isomorphisms $\mathrm{A} \cong \mathrm{V}_{0,2}$ and $\mathrm{C} \cong \mathrm{V}_{1,1}$.

Notation: We let $b: V \rightarrow V^{*}$ via $X^{b}:=\langle X, \cdot\rangle$ denote the usual musical (index-lowering) isomorphism induced by the inner product $\langle\cdot, \cdot\rangle$ on $V$, and let

$$
\sharp: V^{*} \rightarrow V
$$

denote its inverse. In the sequel, we let $A^{\sharp}, C^{\sharp} \subset V$ denote the images of $A, C \subset V^{*}$ under the $\sharp$ isomorphism.

\subsubsection{Decomposition of 2-Forms on $V^{*}$}

We now seek to decompose $\Lambda^{2}\left(V^{*}\right)$ into $\mathrm{SO}(4)$-irreducible submodules. As noted in $\S 2.1$ above, $\Lambda^{2}\left(V^{*}\right)$ splits into $\mathrm{G}_{2}$-irreducible submodules as

$$
\Lambda^{2}\left(V^{*}\right) \cong \Lambda_{7}^{2} \oplus \Lambda_{14}^{2}
$$

On the other hand, using $V^{*}=\mathrm{A} \oplus \mathrm{C}$, we may also decompose $\Lambda^{2}\left(V^{*}\right)$ as

$$
\Lambda^{2}\left(V^{*}\right) \cong \Lambda^{2}(\mathrm{~A}) \oplus(\mathrm{A} \otimes \mathrm{C}) \oplus \Lambda_{+}^{2}(\mathrm{C}) \oplus \Lambda_{-}^{2}(\mathrm{C}) .
$$

We will refine both decompositions (2.6) and (2.7) into $\mathrm{SO}(4)$-submodules.

To begin, note first that as $\mathrm{SO}(4)$-modules, we have that $\Lambda^{2}(\mathrm{~A}) \cong \Lambda_{+}^{2}(\mathrm{C}) \cong \mathrm{V}_{0,2}$ and $\Lambda_{-}^{2}(\mathrm{C}) \cong$ $\mathrm{V}_{2,0}$ are irreducible. Thus, it remains only to decompose $\Lambda_{7}^{2}, \Lambda_{14}^{2}$, and $\mathrm{A} \otimes \mathrm{C}$.

Definition 2.8. We define

$$
\begin{aligned}
\left(\Lambda_{7}^{2}\right)_{\mathrm{A}} & :=\Lambda_{7}^{2} \cap\left(\Lambda^{2}(\mathrm{~A}) \oplus \Lambda^{2}(\mathrm{C})\right)=\left\{\iota_{X} \phi_{0}: X \in \mathrm{A}^{\sharp}\right\} \\
\left(\Lambda_{7}^{2}\right)_{\mathrm{C}} & :=\Lambda_{7}^{2} \cap(\mathrm{A} \otimes \mathrm{C})=\left\{\iota_{X} \phi_{0}: X \in \mathrm{C}^{\sharp}\right\} \\
\left(\Lambda_{14}^{2}\right)_{\mathrm{A}} & :=\Lambda_{14}^{2} \cap\left(\Lambda^{2}(\mathrm{~A}) \oplus \Lambda_{+}^{2}(\mathrm{C})\right) \\
\left(\Lambda_{14}^{2}\right)_{1,3} & :=\Lambda_{14}^{2} \cap(\mathrm{A} \otimes \mathrm{C}) \\
\left(\Lambda_{14}^{2}\right)_{2,0} & :=\Lambda_{14}^{2} \cap \Lambda_{-}^{2}(\mathrm{C}) .
\end{aligned}
$$

The reader can check that, in fact, $\Lambda_{-}^{2}(\mathrm{C}) \subset \Lambda_{14}^{2}$, so that $\left(\Lambda_{14}^{2}\right)_{2,0}=\Lambda_{14}^{2} \cap \Lambda_{-}^{2}(\mathrm{C})=\Lambda_{-}^{2}(\mathrm{C})$.

Consider the $\mathrm{SO}(4)$-module isomorphism

$$
\begin{aligned}
& L: \mathrm{A} \rightarrow\left(\Lambda_{7}^{2}\right)_{\mathrm{A}} \\
& L(\alpha)=\iota_{\alpha^{\sharp}} \phi_{0}=*\left(\alpha \wedge * \phi_{0}\right) .
\end{aligned}
$$

For $\beta \in \Lambda^{2}\left(V^{*}\right)$, write $\beta=\left.\beta\right|_{\Lambda^{2}(\mathrm{~A})}+\left.\beta\right|_{\mathbf{A} \otimes \mathbf{C}}+\left.\beta\right|_{\Lambda^{2}(\mathrm{C})}$, where $\left.\beta\right|_{E} \in E$ for $E \in\left\{\Lambda^{2}(\mathrm{~A}), \mathrm{A} \otimes \mathrm{C}, \Lambda^{2}(\mathrm{C})\right\}$. Define $\mathrm{SO}(4)$-equivariant maps

$$
\begin{array}{ll}
L_{\mathrm{A}}: \mathrm{A} \rightarrow \Lambda^{2}(\mathrm{~A}) & L_{\mathrm{C}}: \mathrm{A} \rightarrow \Lambda_{+}^{2}(\mathrm{C}) \\
L_{\mathrm{A}}(\alpha)=\left.L(\alpha)\right|_{\Lambda^{2}(\mathrm{~A})} & L_{\mathrm{C}}(\alpha)=\left.L(\alpha)\right|_{\Lambda^{2}(\mathrm{C})}
\end{array}
$$


It is straightforward to check that $L_{\mathrm{A}}$ and $L_{\mathrm{C}}$ are well-defined $\mathrm{SO}(4)$-module isomorphisms, and that $L_{\mathrm{A}}=*_{\mathrm{A}}$ coincides with the usual Hodge star operator on A. Finally, we define the map

$$
\begin{aligned}
& W: \mathrm{A} \rightarrow\left(\Lambda_{14}^{2}\right)_{\mathrm{A}} \\
& W(\alpha)=2 L_{\mathrm{A}}(\alpha)-L_{\mathrm{C}}(\alpha)
\end{aligned}
$$

Again, the reader can check that $W$ is a well-defined $\mathrm{SO}(4)$-module isomorphism. We caution that the maps $L, L_{\mathrm{C}}$, and $W$ are not isometries.

Lemma 2.9. The decompositions

$$
\begin{aligned}
\Lambda_{7}^{2} & =\left(\Lambda_{7}^{2}\right)_{\mathrm{A}} \oplus\left(\Lambda_{7}^{2}\right)_{\mathrm{C}} \\
\Lambda_{14}^{2} & =\left(\Lambda_{14}^{2}\right)_{\mathrm{A}} \oplus\left(\Lambda_{14}^{2}\right)_{1,3} \oplus\left(\Lambda_{14}^{2}\right)_{2,0} \\
\mathrm{~A} \otimes \mathrm{C} & =\left(\Lambda_{7}^{2}\right)_{\mathrm{C}} \oplus\left(\Lambda_{14}^{2}\right)_{1,3}
\end{aligned}
$$

consist of $S O(4)$-irreducible submodules.

Thus, the decomposition

$$
\Lambda^{2}(V)=\left[\left(\Lambda_{7}^{2}\right)_{\mathrm{A}} \oplus\left(\Lambda_{7}^{2}\right)_{\mathrm{C}}\right] \oplus\left[\left(\Lambda_{14}^{2}\right)_{\mathrm{A}} \oplus\left(\Lambda_{14}^{2}\right)_{1,3} \oplus\left(\Lambda_{14}^{2}\right)_{2,0}\right]
$$

is SO(4)-irreducible and refines (2.6), while

$$
\Lambda^{2}(V)=\Lambda^{2}(\mathrm{~A}) \oplus\left[\left(\Lambda_{7}^{2}\right)_{\mathrm{C}} \oplus\left(\Lambda_{14}^{2}\right)_{1,3}\right] \oplus \Lambda_{+}^{2}(\mathrm{C}) \oplus \Lambda_{-}^{2}(\mathrm{C})
$$

is $S O(4)$-irreducible and refines (2.7).

Proof. The decomposition (2.8) follows from the isomorphism $V \rightarrow \Lambda_{7}^{2}, X \mapsto \iota_{X}\left(\varphi_{0}\right)$ and the irreducible decomposition $V \cong \mathrm{A} \oplus \mathrm{C}$.

By a dimension count, the $\mathrm{SO}(4)$-invariant subspace $\Lambda_{14}^{2}$ of $\Lambda^{2}\left(V^{*}\right)$ must be isomorphic to the $\mathrm{SO}(4)$-module $\mathrm{V}_{0,2} \oplus \mathrm{V}_{2,0} \oplus \mathrm{V}_{1,3}$, while, by the Clebsch-Gordan formula, the space $\mathrm{A} \otimes \mathrm{C}$ is isomorphic to $\bigvee_{1,3} \oplus \mathrm{V}_{1,1}$. It follows from Schur's lemma that the space $\left(\Lambda_{14}^{2}\right)_{1,3}$ is isomorphic to the $\mathrm{SO}(4)$-module $\mathrm{V}_{1,3}$. The space $\left(\Lambda_{14}^{2}\right)_{\mathrm{A}}$ is the image of $\mathrm{A}$ under the isomorphism $W$ defined above, so it is an irreducible $\mathrm{SO}(4)$-module, while the space $\left(\Lambda_{14}^{2}\right)_{2,0}$ is isomorphic to $\Lambda_{-}^{2}(\mathrm{C}) \cong \mathrm{V}_{2,0}$ so it is an irreducible $\mathrm{SO}(4)$-module. Thus, the decomposition (2.9) consists of irreducible $\mathrm{SO}(4)$-modules.

To see that (2.10) is an irreducible decomposition, note that we have already shown that both $\left(\Lambda_{7}^{2}\right)_{\mathrm{c}}$ and $\left(\Lambda_{14}^{2}\right)_{1,3}$ are irreducible $\mathrm{SO}(4)$-modules.

Definition 2.10. We define the map

$$
\begin{aligned}
\natural:\left(\Lambda_{14}^{2}\right)_{\mathrm{A}} & \rightarrow \mathrm{A}^{\sharp} \\
\beta & \mapsto \beta^{\natural}=\sqrt{6}\left(W^{-1}(\beta)\right)^{\sharp}
\end{aligned}
$$

The map $\downarrow$ is an $\mathrm{SO}(4)$-module isomorphism, and (because of the factor of $\sqrt{6}$ ) an isometry with respect to the inner product $(2.1)$ on $\Lambda^{2}\left(V^{*}\right)$.

\subsubsection{Decomposition of the Quadratic Forms on $V^{*}$}

Before turning to the decomposition of $\Lambda^{3}\left(V^{*}\right)$, we take a moment to decompose $\operatorname{Sym}^{2}\left(V^{*}\right)$ into $\mathrm{SO}(4)$-irreducible pieces. To this end, we first use $V^{*}=\mathrm{A} \oplus \mathrm{C}$ to split

$$
\operatorname{Sym}^{2}\left(V^{*}\right) \cong \mathbb{R I d}_{\mathrm{A}} \oplus \operatorname{Sym}_{0}^{2}(\mathrm{~A}) \oplus(\mathrm{A} \otimes \mathrm{C}) \oplus \mathbb{R I d}_{\mathrm{C}} \oplus \operatorname{Sym}_{0}^{2}(\mathrm{C}) .
$$


Each of these summands is $\mathrm{SO}(4)$-irreducible, with the exception of $\mathrm{A} \otimes \mathrm{C}$, which splits into irreducible summands as

$$
\mathrm{A} \otimes \mathrm{C} \cong(\mathrm{A} \otimes \mathrm{C})_{1,3} \oplus(\mathrm{A} \otimes \mathrm{C})_{\mathrm{C}}
$$

where $(A \otimes C)_{1,3}$ and $(A \otimes C)_{C}$ are submodules isomorphic to $\mathrm{V}_{1,3}$ and $\mathrm{C}$, respectively.

Note that we are employing a slight abuse of notation. That is, in $\S 2.2 .1$ we used $A \otimes C$ to denote a submodule of $\Lambda^{2}\left(V^{*}\right)$, whereas here in $\S 2.2 .2$ we are using the same symbol $\mathrm{A} \otimes \mathrm{C}$ to denote a submodule of $\operatorname{Sym}^{2}\left(V^{*}\right)$. Abstractly, these two $\mathrm{SO}(4)$-modules are isomorphic, as are their irreducible summands. By Schur's Lemma, there is a one-dimensional family of SO(4)-module isomorphisms $\left(\Lambda_{7}^{2}\right)_{\mathrm{C}} \cong(\mathrm{A} \otimes \mathrm{C})_{\mathrm{C}}$ and $\left(\Lambda_{14}^{2}\right)_{1,3} \cong(\mathrm{A} \otimes \mathrm{C})_{1,3}$.

For computations, we will make use of the particular $\mathrm{SO}(4)$-module isomorphism

$$
\mathrm{s}:\left(\Lambda_{7}^{2}\right)_{\mathrm{C}} \rightarrow(\mathrm{A} \otimes \mathrm{C})_{\mathrm{C}}
$$

defined as follows. In terms of a basis $\left\{e^{1}, \ldots, e^{6}\right\}$ of $V^{*}$ with $\mathrm{A}=\operatorname{span}\left(e^{1}, e^{2}, e^{3}\right)$ and $\mathrm{C}=$ $\operatorname{span}\left(e^{4}, e^{5}, e^{6}\right)$, the map s will formally replace $\wedge$ symbols with $\circ$ symbols in each $e^{i} \wedge e^{j}$ term with $i<j$. So, for example,

$$
\mathbf{s}\left(-e^{15}-e^{26}+e^{37}\right)=-e^{1} \circ e^{5}-e^{2} \circ e^{6}+e^{3} \circ e^{7} .
$$

Finally, we remark that $\operatorname{Sym}_{0}^{2}\left(V^{*}\right)$ decomposes into irreducible $\mathrm{SO}(4)$-modules as

$$
\operatorname{Sym}_{0}^{2}\left(V^{*}\right)=\operatorname{Sym}_{0}^{2}(\mathrm{~A}) \oplus(\mathrm{A} \otimes \mathrm{C})_{1,3} \oplus(\mathrm{A} \otimes \mathrm{C})_{\mathrm{C}} \oplus \operatorname{Sym}_{0}^{2}(\mathrm{C}) \oplus \mathbb{R} E_{0},
$$

where

$$
E_{0}=\operatorname{diag}(4,4,4,-3,-3,-3,-3) \in \operatorname{Sym}_{0}^{2}\left(V^{*}\right) .
$$

\subsubsection{Decomposition of 3-Forms on $V^{*}$}

We now turn to $\Lambda^{3}\left(V^{*}\right)$. As noted in $\S 2.1, \Lambda^{3}\left(V^{*}\right)$ splits into $\mathrm{G}_{2}$-irreducible submodules as

$$
\Lambda^{3}\left(V^{*}\right) \cong \Lambda_{1}^{3} \oplus \Lambda_{7}^{3} \oplus \Lambda_{27}^{3}
$$

The summand $\Lambda_{1}^{3} \cong \mathbb{R}$ is $\mathrm{SO}(4)$-irreducible, but the summands $\Lambda_{7}^{3}$ and $\Lambda_{27}^{3}$ are not.

On the other hand, using $V^{*} \cong \mathrm{A} \oplus \mathrm{C}$, we also have the decomposition:

$$
\Lambda^{3}\left(V^{*}\right) \cong \Lambda^{3}(\mathrm{~A}) \oplus\left(\Lambda^{2}(\mathrm{~A}) \otimes \mathrm{C}\right) \oplus\left(\mathrm{A} \otimes \Lambda_{+}^{2}(\mathrm{C})\right) \oplus\left(\mathrm{A} \otimes \Lambda_{-}^{2}(\mathrm{C})\right) \oplus \Lambda^{3}(\mathrm{C}) .
$$

Three of these summands are $\mathrm{SO}(4)$-irreducible, namely $\Lambda^{3}(\mathrm{~A}) \cong \mathrm{V}_{0,0}$ and $\mathrm{A} \otimes \Lambda_{-}^{2}(\mathrm{C}) \cong \mathrm{V}_{2,2}$ and $\Lambda^{3}(\mathrm{C}) \cong \mathrm{V}_{1,1}$. Meanwhile, the second and third summands $\Lambda^{2}(\mathrm{~A}) \otimes \mathrm{C}$ and $\mathrm{A} \otimes \Lambda_{+}^{2}(\mathrm{C})$ are not.

As in $\S 2.2 .1$ above, we will refine both (2.15) and (2.16) into $\mathrm{SO}(4)$-irreducible submodules, though only the refinement of (2.15) will be used in this work. We begin with (2.15).

Definition 2.11. Recall the isomorphism i: $\operatorname{Sym}_{0}^{2}\left(V^{*}\right) \rightarrow \Lambda_{27}^{3}$ of (2.2) and recall the $\operatorname{SO}(4)$ irreducible splitting of $\operatorname{Sym}_{0}^{2}\left(V^{*}\right)$ given in (2.13). We define

$$
\begin{array}{lll}
\left(\Lambda_{7}^{3}\right)_{\mathrm{A}}:=\left\{*\left(\alpha \wedge \phi_{0}\right): \alpha \in \mathrm{A}\right\} & \left(\Lambda_{27}^{3}\right)_{0,0}:=\mathrm{i}\left(E_{0}\right) & \left(\Lambda_{27}^{3}\right)_{1,3}:=\mathrm{i}\left((\mathrm{A} \otimes \mathrm{C})_{1,3}\right) \\
\left(\Lambda_{7}^{3}\right)_{\mathrm{C}}:=\left\{*\left(\alpha \wedge \phi_{0}\right): \alpha \in \mathrm{C}\right\} & \left(\Lambda_{27}^{3}\right)_{0,4}:=\mathrm{i}\left(\operatorname{Sym}_{0}^{2}(\mathrm{~A})\right) & \left(\Lambda_{27}^{3}\right)_{\mathrm{C}}:=\mathrm{i}\left((\mathrm{A} \otimes \mathrm{C})_{\mathrm{C}}\right) \\
& \left(\Lambda_{27}^{3}\right)_{2,2}:=\mathrm{i}\left(\operatorname{Sym}_{0}^{2}(\mathrm{C})\right) &
\end{array}
$$


Lemma 2.12. The decompositions

$$
\begin{aligned}
\Lambda_{7}^{3} & =\left(\Lambda_{7}^{3}\right)_{\mathrm{A}} \oplus\left(\Lambda_{7}^{3}\right)_{\mathrm{C}} \\
\Lambda_{27}^{3} & =\left(\Lambda_{27}^{3}\right)_{0,0} \oplus\left(\Lambda_{27}^{3}\right)_{0,4} \oplus\left(\Lambda_{27}^{3}\right)_{2,2} \oplus\left(\Lambda_{27}^{3}\right)_{1,3} \oplus\left(\Lambda_{27}^{3}\right)_{\mathrm{C}}
\end{aligned}
$$

consist of $S O(4)$-irreducible submodules.

Definition 2.13. Recall the isomorphisms i: $\operatorname{Sym}_{0}^{2}\left(V^{*}\right) \rightarrow \Lambda_{27}^{3}$ of $(2.2)$ and $\mathrm{s}:\left(\Lambda_{7}^{2}\right)_{\mathrm{C}} \rightarrow(\mathrm{A} \otimes \mathrm{C})_{\mathrm{C}}$ of (2.12). We define $\dagger:\left(\Lambda_{27}^{3}\right)_{0,0} \rightarrow \mathbb{R}$ to be the unique vector space isomorphism for which

$$
\left[\mathrm{i}\left(E_{0}\right)\right]^{\dagger}=4 \sqrt{42}
$$

where $E_{0}$ is as in (2.14). The map $\dagger$ is an isometry (due to the choice of $4 \sqrt{42}$ ) with respect to the inner products $(2.1)$.

We will also need the composition of $\mathrm{SO}(4)$-module isomorphisms

$$
\begin{aligned}
& \mathrm{C}^{\sharp} \rightarrow\left(\Lambda_{7}^{2}\right)_{\mathrm{C}} \rightarrow\left(\Lambda_{27}^{3}\right) \mathrm{c} \\
& X \mapsto \iota_{X} \phi_{0} \mapsto \frac{1}{2 \sqrt{3}}(\mathrm{i} \circ \mathrm{s})\left(\iota_{X} \phi_{0}\right) .
\end{aligned}
$$

This map is an isometry due to the factor of $\frac{1}{2 \sqrt{3}}$. We denote the inverse of this isometric isomorphism by

$$
\ddagger:\left(\Lambda_{27}^{3}\right)_{\mathrm{C}} \rightarrow \mathrm{C}^{\sharp}
$$

Remark. Extend the isomorphism $L_{\mathrm{C}}: \mathrm{A} \rightarrow \Lambda_{+}^{2}(\mathrm{C})$ to an isomorphism $L_{\mathrm{C}}: \mathrm{A} \otimes \mathrm{A} \rightarrow \mathrm{A} \otimes \Lambda_{+}^{2}(\mathrm{C})$ by the identity on the first $A$-factor, and split $A \otimes A=\mathbb{R} \oplus \operatorname{Sym}_{0}^{2}(A) \oplus \Lambda^{2}(A)$. Extend the Hodge star operator $*_{\mathrm{A}}: \mathrm{A} \rightarrow \Lambda^{2}(\mathrm{~A})$ to an isomorphism $*_{\mathrm{A}}: \mathrm{A} \otimes \mathrm{C} \rightarrow \Lambda^{2}(\mathrm{~A}) \otimes \mathrm{C}$ by the identity on the $\mathrm{C}$-factor, and recall the decomposition $\mathrm{A} \otimes \mathrm{C}=\left(\Lambda_{7}^{2}\right)_{\mathrm{C}} \oplus\left(\Lambda_{14}^{2}\right)_{1,3}$.

Defining

$$
\begin{aligned}
\left(\Lambda^{2}(\mathrm{~A}) \otimes \mathrm{C}\right)_{\mathrm{C}}:=*_{\mathrm{A}}\left[\left(\Lambda_{7}^{2}\right)_{\mathrm{C}}\right] & \left(\mathrm{A} \otimes \Lambda_{+}^{2}(\mathrm{C})\right)_{0,0}:=L_{\mathrm{C}}(\mathbb{R}) \\
\left(\Lambda^{2}(\mathrm{~A}) \otimes \mathrm{C}\right)_{1,3}:=*_{\mathrm{A}}\left[\left(\Lambda_{14}^{2}\right)_{1,3}\right] & \left(\mathrm{A} \otimes \Lambda_{+}^{2}(\mathrm{C})\right)_{0,4}:=L_{\mathrm{C}}\left(\operatorname{Sym}_{0}^{2}(\mathrm{~A})\right) \\
& \left(\mathrm{A} \otimes \Lambda_{+}^{2}(\mathrm{C})\right)_{\mathrm{A}}:=L_{\mathrm{C}}\left(\Lambda^{2}(\mathrm{~A})\right)
\end{aligned}
$$

we obtain decompositions

$$
\begin{aligned}
\Lambda^{2}(\mathrm{~A}) \otimes \mathrm{C} & =\left(\Lambda^{2}(\mathrm{~A}) \otimes \mathrm{C}\right)_{\mathrm{C}} \oplus\left(\Lambda^{2}(\mathrm{~A}) \otimes \mathrm{C}\right)_{1,3} \\
\mathrm{~A} \otimes \Lambda_{+}^{2}(\mathrm{C}) & =\left(\mathrm{A} \otimes \Lambda_{+}^{2}(\mathrm{C})\right)_{0,0} \oplus\left(\mathrm{A} \otimes \Lambda_{+}^{2}(\mathrm{C})\right)_{0,4} \oplus\left(\mathrm{A} \otimes \Lambda_{+}^{2}(\mathrm{C})\right)_{\mathrm{A}}
\end{aligned}
$$

consisting of $\mathrm{SO}(4)$-irreducible submodules.

Remark. The reader can check that some of the above submodules of $\Lambda^{3}\left(V^{*}\right)$ are, in fact, equal to one another. Namely, we have the equalities

$$
\begin{aligned}
\mathrm{A} \otimes \Lambda_{-}^{2}(\mathrm{C}) & =\left(\Lambda_{27}^{3}\right)_{2,2} & \left(\mathrm{~A} \otimes \Lambda_{+}^{2}(\mathrm{C})\right)_{0,4} & =\left(\Lambda_{27}^{3}\right)_{0,4} \\
\left(\Lambda^{2}(\mathrm{~A}) \otimes \mathrm{C}\right)_{1,3} & =\left(\Lambda_{27}^{3}\right)_{1,3} & \left(\mathrm{~A} \otimes \Lambda_{+}^{2}(\mathrm{C})\right)_{\mathrm{A}} & =\left(\Lambda_{7}^{3}\right)_{\mathrm{A}}
\end{aligned}
$$




\subsection{The Refined Torsion Forms}

Let $\left(M^{7}, \varphi\right)$ be a 7 -manifold equipped with a $\mathrm{G}_{2}$-structure $\varphi$. Fix a point $x \in M$, choose an arbitrary associative 3-plane $A^{\sharp} \subset T_{x} M$, and let $C^{\sharp} \subset T_{x} M$ denote its orthogonal coassociative 4plane. Our purpose in this section is to understand how the torsion of the $\mathrm{G}_{2}$-structure decomposes with respect to the splitting

$$
T_{x} M=\mathrm{A}^{\sharp} \oplus \mathrm{C}^{\sharp} .
$$

In $§ 2.3 .1$, we use the decompositions of Lemmas 2.9 and 2.12 to break the torsion forms $\tau_{0}, \tau_{1}, \tau_{2}, \tau_{3}$ into $\mathrm{SO}(4)$-irreducible pieces called refined torsion forms. Separately, in $\S 2.3 .2$, we set up the $\mathrm{G}_{2}$-coframe bundle $\pi: F_{\mathrm{G}_{2}} \rightarrow M$ following [5], repackaging the original $\mathrm{G}_{2}$ torsion forms $\tau_{0}, \tau_{1}, \tau_{2}, \tau_{3}$ as a matrix-valued function

$$
T=\left(T_{i j}\right): F_{\mathrm{G}_{2}} \rightarrow \operatorname{Mat}_{7 \times 7}(\mathbb{R}) \simeq \mathbb{R}^{49}
$$

Finally, in $\S 2.3 .3$, we express the functions $T_{i j}$ in terms of the (pullbacks of the) refined torsion forms.

\subsubsection{The Refined Torsion Forms in a Local SO(4)-Frame}

Fix $x \in M$ and split $T_{x}^{*} M=\mathrm{A} \oplus \mathrm{C}$ as above. All of our calculations in this subsection will be done pointwise, and we will suppress reference to $x \in M$. By Lemmas 2.9 and 2.12, the torsion forms $\tau_{0}, \tau_{1}, \tau_{2}, \tau_{3}$ decompose into $\mathrm{SO}(4)$-irreducible pieces as

$$
\begin{aligned}
& \tau_{0}=\tau_{0} \\
& \tau_{1}=\left(\tau_{1}\right)_{\mathbf{A}}+\left(\tau_{1}\right)_{\mathbf{C}} \\
& \tau_{2}=\left(\tau_{2}\right)_{\mathbf{A}}+\left(\tau_{2}\right)_{1,3}+\left(\tau_{2}\right)_{2,0} \\
& \tau_{3}=\left(\tau_{3}\right)_{0,0}+\left(\tau_{3}\right)_{0,4}+\left(\tau_{3}\right)_{2,2}+\left(\tau_{3}\right)_{1,3}+\left(\tau_{3}\right)_{\mathbf{C}}
\end{aligned}
$$

where here

$$
\begin{array}{llr}
\left(\tau_{1}\right)_{\mathbf{A}} \in \mathbf{A} & \left(\tau_{2}\right)_{\mathbf{A}} \in\left(\Lambda_{14}^{2}\right)_{\mathbf{A}} & \left(\tau_{3}\right)_{0,0} \in\left(\Lambda_{27}^{3}\right)_{0,0} \\
\left(\tau_{1}\right)_{\mathbf{C}} \in \mathbf{C} & \left(\tau_{2}\right)_{1,3} \in\left(\Lambda_{14}^{2}\right)_{1,3} & \left(\tau_{3}\right)_{0,4} \in\left(\Lambda_{27}^{3}\right)_{0,4} \\
& \left(\tau_{2}\right)_{2,0} \in\left(\Lambda_{14}^{2}\right)_{2,0} & \left(\tau_{3}\right)_{2,2} \in\left(\Lambda_{27}^{3}\right)_{2,2} \\
& \left(\tau_{3}\right)_{1,3} \in\left(\Lambda_{27}^{3}\right)_{1,3} \\
& \left(\tau_{3}\right)_{\mathrm{C}} \in\left(\Lambda_{27}^{3}\right)_{\mathrm{C}}
\end{array}
$$

We refer to $\tau_{0},\left(\tau_{1}\right)_{\mathrm{A}},\left(\tau_{1}\right)_{\mathrm{C}}, \ldots,\left(\tau_{3}\right)_{\mathrm{C}}$ as the refined torsion forms of the $\mathrm{G}_{2}$-structure at $x$ relative to the splitting $T_{x}^{*} M=\mathrm{A} \oplus \mathrm{C}$.

We now move to express the refined torsion forms in terms of a local $\mathrm{SO}(4)$-frame, for which we will need explicit bases of $\left(\Lambda_{14}^{2}\right)_{\mathrm{A}}, \ldots,\left(\Lambda_{27}^{3}\right)_{\mathrm{C}}$. To that end, let $\left\{e_{1}, \ldots, e_{7}\right\}$ be an orthonormal basis for $T_{x} M$ for which $A^{\sharp}=\operatorname{span}\left(e_{1}, e_{2}, e_{3}\right)$ and $C^{\sharp}=\operatorname{span}\left(e_{4}, e_{5}, e_{6}, e_{7}\right)$. Let $\left\{e^{1}, \ldots, e^{7}\right\}$ denote the dual basis for $T_{x}^{*} M$.

Index Ranges: We will employ the following index ranges: $1 \leq p, q \leq 3$ and $4 \leq \alpha, \beta \leq 7$ and $1 \leq i, j, k, \ell, m \leq 7$ and $1 \leq \delta \leq 8$ and $1 \leq a \leq 5$. 
Definition 2.14. Define the 2-forms

$$
\begin{array}{llll}
\Upsilon_{1}=e^{45}+e^{67} & \Omega_{1}=e^{45}-e^{67} & \Delta_{1}=e^{17}+e^{24} & \Delta_{5}=e^{16}+e^{34} \\
\Upsilon_{2}=e^{46}-e^{57} & \Omega_{2}=e^{46}+e^{57} & \Delta_{2}=e^{16}+e^{25} & \Delta_{6}=-e^{17}+e^{35} \\
\Upsilon_{3}=-e^{47}-e^{56} & \Omega_{3}=e^{47}-e^{56} & \Delta_{3}=-e^{15}+e^{26} & \Delta_{7}=-e^{14}+e^{36} \\
& & \Delta_{4}=-e^{14}+e^{36} & \Delta_{8}=e^{15}+e^{37}
\end{array}
$$

We also define

$$
\Gamma_{p}=2 *_{\mathrm{A}} e^{p}-\Upsilon_{p}
$$

(no summation).

Lemma 2.15. We have that:

(a) $\left\{\Gamma_{1}, \Gamma_{2}, \Gamma_{3}\right\}$ is a basis of $\left(\Lambda_{14}^{2}\right)_{\mathrm{A}}$.

(b) $\left\{\Delta_{1}, \ldots, \Delta_{8}\right\}$ is a basis of $\left(\Lambda_{14}^{2}\right)_{1,3}$.

(c) $\left\{\Omega_{1}, \Omega_{2}, \Omega_{3}\right\}$ is a basis of $\left(\Lambda_{14}^{2}\right)_{2,0}$.

Definition 2.16. Define the 3-forms

$$
\begin{aligned}
\phi_{\mathrm{A}} & =e^{123} & \lambda_{p q} & =e^{p} \wedge \Omega_{q} \\
\phi_{\mathrm{C}} & =\sum e^{p} \wedge \Upsilon_{p} & \nu_{\alpha} & =(\mathrm{i} \circ \mathrm{s})\left(\iota_{e_{\alpha}} \phi_{0}\right)
\end{aligned}
$$

and

$$
\begin{aligned}
& \kappa_{1}=e^{1} \wedge \Upsilon_{2}-e^{2} \wedge \Upsilon_{1} \\
& \mu_{1}=e^{237}+e^{314} \\
& \kappa_{2}=e^{1} \wedge \Upsilon_{3}+e^{3} \wedge \Upsilon_{1} \\
& \mu_{2}=e^{236}+e^{315} \\
& \kappa_{3}=e^{2} \wedge \Upsilon_{3}+e^{3} \wedge \Upsilon_{2} \\
& \mu_{3}=-e^{235}+e^{316} \\
& \mu_{5}=e^{236}+e^{124} \\
& \kappa_{4}=e^{1} \wedge \Upsilon_{1}-e^{2} \wedge \Upsilon_{2} \\
& \mu_{4}=-e^{234}+e^{317} \\
& \mu_{6}=-e^{237}+e^{125} \\
& \kappa_{5}=e^{2} \wedge \Upsilon_{2}-e^{3} \wedge \Upsilon_{3} \text {. } \\
& \mu_{7}=-e^{234}+e^{126} \\
& \mu_{8}=e^{235}+e^{127}
\end{aligned}
$$

Note that $\varphi=\phi_{\mathrm{A}}+\phi_{\mathrm{C}}$.

Lemma 2.17. We have that:

(a) $\left\{6 \phi_{\mathrm{A}}-\phi_{\mathrm{C}}\right\}$ is a basis of $\left(\Lambda_{27}^{3}\right)_{0,0}$

(b) $\left\{\kappa_{1}, \kappa_{2}, \kappa_{3}, \kappa_{4}, \kappa_{5}\right\}$ is a basis of $\left(\Lambda_{27}^{3}\right)_{0,4}$

(c) $\left\{\lambda_{p q}: 1 \leq p, q \leq 3\right\}$ is a basis of $\left(\Lambda_{27}^{3}\right)_{2,2}$

(d) $\left\{\mu_{1}, \ldots, \mu_{8}\right\}$ is basis of $\left(\Lambda_{27}^{3}\right)_{1,3}$

(e) $\left\{\nu_{4}, \nu_{5}, \nu_{6}, \nu_{7}\right\}$ is a basis of $\left(\Lambda_{27}^{3}\right) \mathrm{C}$

We now express $\left(\tau_{1}\right)_{\mathrm{A}},\left(\tau_{1}\right)_{\mathrm{C}}$, etc., in terms of the above bases. That is, we define functions $A_{p}, B_{\alpha}$ and $C_{p}, D_{\delta}, E_{p}$ and $F, G_{a}, J_{p q}, L_{\delta}, M_{\alpha}$ by:

$$
\begin{array}{rlrl}
\left(\tau_{1}\right)_{\mathrm{A}}=6 A_{p} e^{p} & \left(\tau_{2}\right)_{\mathrm{A}}=12 C_{p} \Gamma_{p} & \left(\tau_{3}\right)_{0,0}=12 F\left(6 \phi_{\mathrm{A}}-\phi_{\mathrm{C}}\right) \\
\left(\tau_{1}\right)_{\mathrm{C}}=6 B_{\alpha} e^{\alpha} & \left(\tau_{2}\right)_{1,3}=12 D_{\delta} \Delta_{\delta} & \left(\tau_{3}\right)_{0,4}=6 G_{a} \kappa_{a} \\
\left(\tau_{2}\right)_{2,0}=12 E_{p} \Omega_{p} & \left(\tau_{3}\right)_{2,2}=12 J_{p q} \lambda_{p q} \\
\left(\tau_{3}\right)_{1,3}=12 L_{\delta} \mu_{\delta} \\
\left(\tau_{3}\right)_{\mathrm{C}}=6 M_{\alpha} \nu_{\alpha}
\end{array}
$$

The various factors of 6 and 12 are included simply for the sake of clearing future denominators. 
Note that the bases of Lemmas 2.15 and 2.17 are orthogonal but not orthonormal with respect to the inner product $(2.1)$ on $\Lambda^{k}\left(V^{*}\right)$. Indeed, we have:

$$
\begin{aligned}
& \left\|\Gamma_{p}\right\|=\sqrt{6} \\
& \left\|\Omega_{p}\right\|=\sqrt{2} \\
& \left\|\mu_{\delta}\right\|=\sqrt{2} \\
& \left\|\kappa_{a}\right\|=2 \\
& \left\|\Delta_{\delta}\right\|=\sqrt{2} \\
& \left\|6 \phi_{\mathrm{A}}-\phi_{\mathrm{C}}\right\|=\sqrt{42} \\
& \left\|\nu_{\alpha}\right\|=2 \sqrt{3} \\
& \left\|\lambda_{p q}\right\|=\sqrt{2}
\end{aligned}
$$

Thus, in terms of the isometric isomorphisms (2.5), (2.11), (2.17), (2.18) of $\S 2.2$, we have:

$$
\begin{array}{rlrl}
{\left[\left(\tau_{1}\right)_{\mathrm{A}}\right]^{\sharp}=6 A_{p} e_{p}} & {\left[\left(\tau_{2}\right)_{\mathrm{A}}\right]^{\natural}=12 \sqrt{6} C_{p} e_{p}} & {\left[\left(\tau_{3}\right)_{0,0}\right]^{\dagger}=12 \sqrt{42} \mathrm{~F}} \\
{\left[\left(\tau_{1}\right)_{\mathrm{C}}\right]^{\sharp}=6 B_{\alpha} e_{\alpha}} & {\left[\left(\tau_{3}\right)_{\mathrm{C}}\right]^{\ddagger}=12 \sqrt{3} M_{\alpha} e_{\alpha}}
\end{array}
$$

We will need these for our calculations in $\S 2.4$ and $\S 2.5$.

\subsubsection{The Torsion Functions $T_{i j}$}

Let $\left(M^{7}, \varphi\right)$ be a 7 -manifold with a $\mathrm{G}_{2}$-structure $\varphi$, and let $g_{\varphi}$ denote the underlying Riemannian metric. Let $F_{\mathrm{SO}(7)} \rightarrow M$ denote the oriented orthonormal coframe bundle of $g_{\varphi}$, and let $\omega=\left(\omega^{1}, \ldots, \omega^{7}\right) \in \Omega^{1}\left(F_{\mathrm{SO}(7)} ; \mathbb{R}^{7}\right)$ denote the tautological 1-form. By the Fundamental Lemma of Riemannian Geometry, there exists a unique 1-form $\psi \in \Omega^{1}\left(F_{\mathrm{SO}(7)} ; \mathfrak{s o}(7)\right)$, the Levi-Civita connection form of $g_{\varphi}$, satisfying the First Structure Equation

$$
d \omega=-\psi \wedge \omega
$$

Let $\pi: F_{\mathrm{G}_{2}} \rightarrow M$ denote the $\mathrm{G}_{2}$-coframe bundle of $M$. Restricted to $F_{\mathrm{G}_{2}} \subset F_{\mathrm{SO}(7)}$, the LeviCivita 1 -form $\psi$ is no longer a connection 1-form in general. Indeed, according to the splitting $\mathfrak{s o}(7)=\mathfrak{g}_{2} \oplus \mathbb{R}^{7}$, we have the decomposition

$$
\psi=\theta+2 \gamma
$$

where $\theta=\left(\theta_{i j}\right) \in \Omega^{1}\left(F_{\mathrm{G}_{2}} ; \mathfrak{g}_{2}\right)$ is a connection 1-form (the so-called natural connection of the $\mathrm{G}_{2^{-}}$ structure $\varphi)$ and $\gamma \in \Omega^{1}\left(F_{\mathrm{G}_{2}} ; \mathbb{R}^{7}\right)$ is a $\pi$-semi-basic 1 -form. Here, we are viewing $\mathbb{R}^{7}=\left\{\left(\epsilon_{i j k} v_{k}\right) \in\right.$ $\left.\mathfrak{s o}(7):\left(v_{1}, \ldots, v_{7}\right) \in \mathbb{R}^{7}\right\}$, so that $\gamma$ takes the form

$$
\gamma=\left[\begin{array}{ccc|cccc}
0 & \gamma_{3} & -\gamma_{2} & \gamma_{5} & -\gamma_{4} & \gamma_{7} & -\gamma_{6} \\
-\gamma_{3} & 0 & \gamma_{1} & \gamma_{6} & -\gamma_{7} & -\gamma_{4} & \gamma_{5} \\
\gamma_{2} & -\gamma_{1} & 0 & -\gamma_{7} & -\gamma_{6} & \gamma_{5} & \gamma_{4} \\
\hline-\gamma_{5} & -\gamma_{6} & \gamma_{7} & 0 & \gamma_{1} & \gamma_{2} & -\gamma_{3} \\
\gamma_{4} & \gamma_{7} & \gamma_{6} & -\gamma_{1} & 0 & -\gamma_{3} & -\gamma_{2} \\
-\gamma_{7} & \gamma_{4} & -\gamma_{5} & -\gamma_{2} & \gamma_{3} & 0 & \gamma_{1} \\
\gamma_{6} & -\gamma_{5} & -\gamma_{4} & \gamma_{3} & \gamma_{2} & -\gamma_{1} & 0
\end{array}\right]
$$

Since $\gamma$ is $\pi$-semibasic, we may write

$$
\gamma_{i}=T_{i j} \omega^{j}
$$

for some matrix-valued function $T=\left(T_{i j}\right): F_{\mathrm{G}_{2}} \rightarrow \operatorname{Mat}_{7 \times 7}(\mathbb{R})$. The 1-form $\gamma$, and hence the functions $T_{i j}$, encodes the torsion of the $\mathrm{G}_{2}$-structure. In this notation, the first structure equation reads

$$
d \omega_{i}=-\left(\theta_{i j}+2 \epsilon_{i j k} \gamma_{k}\right) \wedge \omega_{j}
$$


Remark. The reader may wonder how the functions $T_{i j}$ are related to the forms $\tau_{0}, \tau_{1}, \tau_{2}, \tau_{3}$. In [5], Bryant expresses the torsion forms $\tau_{0}, \tau_{1}, \tau_{2}, \tau_{3}$ in terms of $T_{i j}$ as:

$$
\begin{aligned}
& \pi^{*}\left(\tau_{0}\right)=\frac{24}{7} T_{i i} \\
& \pi^{*}\left(\tau_{1}\right)=\epsilon_{i j k} T_{i j} \omega_{k} \\
& \pi^{*}\left(\tau_{2}\right)=4 T_{i j} \omega_{i} \wedge \omega_{j}-\epsilon_{i j k \ell} T_{i j} \omega_{k} \wedge \omega_{\ell} \\
& \pi^{*}\left(\tau_{3}\right)=-\frac{3}{2} \epsilon_{i k \ell}\left(T_{i j}+T_{j i}\right) \omega_{j k \ell}+\frac{18}{7} T_{i i} \sigma .
\end{aligned}
$$

In the next section, we will exhibit a sort of inverse to this, expressing the $T_{i j}$ in terms of the refined torsion forms $\pi^{*}\left(\tau_{0}\right), \pi^{*}\left(\left(\tau_{1}\right)_{\mathrm{A}}\right), \ldots, \pi^{*}\left(\left(\tau_{3}\right)_{\mathrm{C}}\right)$.

\subsubsection{Decomposition of the Torsion Functions}

For our computations in $\S 2.4$ and $\S 2.5$, we will need to express the torsion functions $T_{i j}$ in terms of the functions $A_{p}, B_{\alpha}, \ldots, L_{\delta}, M_{\alpha}$. To this end, we will continue to work on the total space of the $\mathrm{G}_{2}$-coframe bundle $\pi: F_{\mathrm{G}_{2}} \rightarrow M$, pulling back all of the quantities defined on $M$ to $F_{\mathrm{G}_{2}}$. Following common convention, we systematically omit $\pi^{*}$ from the notation, so that (for example) $\pi^{*}\left(\tau_{0}\right)$ will simply be denoted $\tau_{0}$, etc. Note, however, that $\pi^{*}\left(e^{j}\right)=\omega_{j}$.

To begin, recall that the torsion forms $\tau_{0}, \tau_{1}, \tau_{2}, \tau_{3}$ satisfy

$$
\begin{array}{rlrl}
d \varphi & =\tau_{0} * \varphi+3 \tau_{1} \wedge \varphi+* \tau_{3} \\
d * \varphi & = & 4 \tau_{1} \wedge * \varphi+\tau_{2} \wedge \varphi .
\end{array}
$$

Into the left-hand sides, we substitute $\varphi=\frac{1}{6} \epsilon_{i j k} \omega^{i j k}$ and $* \varphi=\frac{1}{24} \epsilon_{i j k \ell} \omega^{i j k \ell}$ and use the first structure equation (2.22) to obtain

$$
\begin{aligned}
\epsilon_{i j k \ell} T_{i m} \omega^{m j k \ell} & =\tau_{0} * \varphi+3 \tau_{1} \wedge \varphi+* \tau_{3} \\
-\epsilon_{i j k} T_{\ell m} \omega^{m \ell i j k} & =\quad 4 \tau_{1} \wedge * \varphi+\tau_{2} \wedge \varphi
\end{aligned}
$$

Into the right-hand sides, we again substitute $\varphi=\frac{1}{6} \epsilon_{i j k} \omega^{i j k}$ and $* \varphi=\frac{1}{24} \epsilon_{i j k \ell} \omega^{i j k \ell}$, as well as the expansions (2.19) and (2.20).

Upon equating coefficients, we obtain a system of $56=\left(\begin{array}{l}7 \\ 4\end{array}\right)+\left(\begin{array}{l}7 \\ 5\end{array}\right)$ linear equations relating the $49=7^{2}$ functions $T_{i j}$ on the left side to the $49=\operatorname{dim}\left(H^{0,2}\left(\mathfrak{g}_{2}\right)\right)$ functions $\tau_{0}, A_{p}, B_{\alpha}, \ldots, L_{\delta}, M_{\alpha}$ on the right side. One can then use a computer algebra system (we have used MAPLE) to solve this linear system for the $T_{i j}$.

We now exhibit the result, taking advantage of the $\mathrm{SO}(4)$-irreducible splitting

$$
\begin{aligned}
\operatorname{Mat}_{7 \times 7}(\mathbb{R}) \cong V^{*} \otimes V^{*} \cong & (\mathrm{A} \otimes \mathrm{A}) \oplus 2(\mathrm{~A} \otimes \mathrm{C}) \oplus(\mathrm{C} \otimes \mathrm{C}) \\
\cong & \left(\Lambda^{2}(\mathrm{~A}) \oplus \operatorname{Sym}_{0}^{2}(\mathrm{~A}) \oplus \mathbb{R}\right) \oplus 2\left((\mathrm{~A} \otimes \mathrm{C})_{1,3} \oplus(\mathrm{A} \otimes \mathrm{C})_{\mathrm{C}}\right) \\
& \oplus\left(\Lambda_{+}^{2}(\mathrm{C}) \oplus \Lambda_{-}^{2}(\mathrm{C}) \oplus \mathbb{R} \oplus \mathrm{Sym}_{0}^{2}(\mathrm{C})\right)
\end{aligned}
$$

to highlight the structure of the solution.

We have

$$
\begin{aligned}
& \frac{1}{2}\left[\begin{array}{ccc}
0 & T_{12}-T_{21} & T_{13}-T_{31} \\
T_{21}-T_{12} & 0 & T_{23}-T_{32} \\
T_{31}-T_{13} & T_{32}-T_{23} & 0
\end{array}\right]=\left[\begin{array}{ccc}
0 & A_{2}+2 C_{3} & -\left(A_{2}+2 C_{2}\right) \\
-\left(A_{3}+2 C_{3}\right) & 0 & A_{1}+2 C_{1} \\
A_{2}+2 C_{2} & -\left(A_{1}+2 C_{1}\right) & 0
\end{array}\right], \\
& \frac{1}{2}\left[\begin{array}{ccc}
2 T_{11} & T_{12}+T_{21} & T_{13}+T_{31} \\
T_{21}+T_{12} & 2 T_{22} & T_{23}+T_{32} \\
T_{31}+T_{13} & T_{32}+T_{23} & 2 T_{33}
\end{array}\right]=-\left[\begin{array}{ccc}
G_{4} & G_{1} & G_{2} \\
G_{1} & G_{5}-G_{4} & G_{3} \\
G_{2} & G_{3} & -G_{5}
\end{array}\right]+\left(-4 F+\frac{1}{24} \tau_{0}\right) \mathrm{Id}_{3},
\end{aligned}
$$


corresponding to $\mathrm{A} \otimes \mathrm{A} \cong \Lambda^{2}(\mathrm{~A}) \oplus \operatorname{Sym}_{0}^{2}(\mathrm{~A}) \oplus \mathbb{R}$ and

$$
\frac{1}{2}\left[\begin{array}{ccc}
T_{41}+T_{14} & T_{42}+T_{24} & T_{43}+T_{34} \\
T_{51}+T_{15} & T_{52}+T_{25} & T_{53}+T_{35} \\
T_{61}+T_{16} & T_{62}+T_{26} & T_{63}+T_{36} \\
T_{71}+T_{17} & T_{72}+T_{27} & T_{73}+T_{37}
\end{array}\right]=\left[\begin{array}{ccc}
L_{4}+L_{7} & -L_{1} & -L_{5} \\
L_{3}-L_{8} & -L_{2} & -L_{6} \\
-L_{2}-L_{5} & -L_{3} & -L_{7} \\
-L_{1}+L_{6} & -L_{4} & -L_{8}
\end{array}\right]+\left[\begin{array}{ccc}
-M_{5} & -M_{6} & M_{7} \\
M_{4} & M_{7} & M_{6} \\
-M_{7} & M_{4} & -M_{5} \\
M_{6} & -M_{5} & -M_{4}
\end{array}\right]
$$

and

$$
\frac{1}{2}\left[\begin{array}{ccc}
T_{41}-T_{14} & T_{42}-T_{24} & T_{43}-T_{34} \\
T_{51}-T_{15} & T_{52}-T_{25} & T_{53}-T_{35} \\
T_{61}-T_{16} & T_{62}-T_{26} & T_{63}-T_{36} \\
T_{71}-T_{17} & T_{72}-T_{27} & T_{73}-T_{37}
\end{array}\right]=\left[\begin{array}{ccc}
D_{4}+D_{7} & -D_{1} & -D_{5} \\
D_{3}-D_{8} & -D_{2} & -D_{6} \\
-D_{2}-D_{5} & -D_{3} & -D_{7} \\
-D_{1}+D_{6} & -D_{4} & -D_{8}
\end{array}\right]+\left[\begin{array}{ccc}
-B_{5} & -B_{6} & B_{7} \\
B_{4} & B_{7} & B_{6} \\
-B_{7} & B_{4} & -B_{5} \\
B_{6} & -B_{5} & -B_{4}
\end{array}\right]
$$

corresponding to $\mathrm{A} \otimes \mathrm{C} \cong(\mathrm{A} \otimes \mathrm{C})_{1,3} \oplus(\mathrm{A} \otimes \mathrm{C})_{\mathrm{C}}$, and

$$
\begin{aligned}
\frac{1}{2}\left[\begin{array}{cccc}
0 & T_{45}-T_{54} & T_{46}-T_{64} & T_{47}-T_{74} \\
T_{54}-T_{45} & 0 & T_{56}-T_{65} & T_{57}-T_{57} \\
T_{64}-T_{46} & T_{65}-T_{56} & 0 & T_{67}-T_{76} \\
T_{74}-T_{47} & T_{75}-T_{57} & T_{76}-T_{67} & 0
\end{array}\right]= & {\left[\begin{array}{cccc}
0 & A_{1}-C_{1} & A_{2}-C_{2} & -A_{3}+C_{3} \\
-A_{1}+C_{1} & 0 & -A_{3}+C_{3} & -A_{2}+C_{2} \\
-A_{2}-C_{2} & A_{3}-C_{3} & 0 & A_{1}-C_{1} \\
A_{3}-C_{3} & A_{2}-C_{2} & -A_{1}+C_{1} & 0
\end{array}\right] } \\
& +\left[\begin{array}{cccc}
0 & E_{1} & E_{2} & E_{3} \\
-E_{1} & 0 & -E_{3} & E_{2} \\
-E_{2} & E_{3} & 0 & -E_{1} \\
-E_{3} & -E_{2} & E_{1} & 0
\end{array}\right]
\end{aligned}
$$

and

$$
\begin{aligned}
\frac{1}{2} & {\left[\begin{array}{cccc}
2 T_{44} & T_{45}+T_{54} & T_{46}+T_{64} & T_{47}+T_{74} \\
T_{54}+T_{45} & 2 T_{55} & T_{56}+T_{65} & T_{57}+T_{75} \\
T_{64}+T_{46} & T_{65} & 2 T_{66} & T_{67}+T_{76} \\
T_{74}+T_{47} & T_{75}+T_{57} & T_{76}+T_{67} & 2 T_{77}
\end{array}\right]=} \\
& {\left[\begin{array}{cccc}
-J_{11}-J_{22}+J_{33} & J_{23}+J_{32} & -J_{13}-J_{31} & J_{12}-J_{21} \\
J_{23}+J_{32} & -J_{11}+J_{22}-J_{33} & -J_{21}-J_{12} & -J_{13}+J_{31} \\
-J_{13}-J_{31} & -J_{12}-J_{21} & J_{11}-J_{22}-J_{33} & -J_{23}+J_{32} \\
J_{12}-J_{21} & -J_{13}+J_{31} & -J_{23}+J_{32} & J_{11}+J_{22}+J_{33}
\end{array}\right]+\left(3 F+\frac{1}{24} \tau_{0}\right) \operatorname{Id}_{4} }
\end{aligned}
$$

corresponding to $\mathrm{C} \otimes \mathrm{C} \cong \Lambda_{+}^{2}(\mathrm{C}) \oplus \Lambda_{-}^{2}(\mathrm{C}) \oplus \mathbb{R} \oplus \operatorname{Sym}_{0}^{2}(\mathrm{C})$.

The above relations are more than we need for this work. In fact, we will only make use of the following relations, which can be read off from the above:

$$
\epsilon_{\alpha \beta p} T_{\beta p}=-3\left(B_{\alpha}+M_{\alpha}\right)
$$

and

$$
T_{44}+T_{55}+T_{66}+T_{77}=3 F+\frac{1}{24} \tau_{0}
$$

and

$$
\begin{array}{r}
-\left(T_{45}-T_{54}\right)-\left(T_{67}-T_{76}\right)=-4\left(A_{1}-C_{1}\right) \\
\left(T_{57}-T_{75}\right)-\left(T_{46}-T_{64}\right)=-4\left(A_{2}-C_{2}\right) \\
\left(T_{47}-T_{74}\right)+\left(T_{56}-T_{65}\right)=-4\left(A_{3}-C_{3}\right) .
\end{array}
$$




\subsection{Mean Curvature of Associative 3-Folds}

In this subsection, we derive a formula (Theorem 2.18) for the mean curvature of an associative 3 -fold in an arbitrary 7 -manifold $(M, \varphi)$ with $\mathrm{G}_{2}$-structure $\varphi$.

We continue with the notation of $\S 2.3$, letting $\pi: F_{\mathrm{G}_{2}} \rightarrow M$ denote the $\mathrm{G}_{2}$-coframe bundle of $M$, and $\omega=\left(\omega_{\mathrm{A}}, \omega_{\mathrm{C}}\right) \in \Omega^{1}\left(F_{\mathrm{G}_{2}} ; \mathrm{A}^{\sharp} \oplus \mathrm{C}^{\sharp}\right)$ denote the tautological 1-form. We remind the reader that $\theta=\left(\theta_{i j}\right) \in \Omega^{1}\left(F_{\mathrm{G}_{2}} ; \mathfrak{g}_{2}\right)$ is the natural connection 1-form, and that $\gamma=\left(\gamma_{i j}\right) \in \Omega^{1}\left(F_{\mathrm{G}_{2}} ; \mathbb{R}^{7}\right)$ is a $\pi$-semibasic 1-form encoding the torsion of $\varphi$. We will continue to write $\gamma_{i j}=\epsilon_{i j k} \gamma_{k}$ and $\gamma_{i}=T_{i j} \omega^{j}$ for $T=\left(T_{i j}\right): F_{\mathrm{G}_{2}} \rightarrow \operatorname{Mat}_{7 \times 7}(\mathbb{R})$.

Let $f: \Sigma^{3} \rightarrow M^{7}$ denote an immersion of an associative 3 -fold into $M$, and let $f^{*}\left(F_{\mathrm{G}_{2}}\right) \rightarrow \Sigma$ denote the pullback bundle. Let $B \subset f^{*}\left(F_{\mathrm{G}_{2}}\right)$ denote the subbundle of coframes adapted to $\Sigma$, i.e., the subbundle whose fiber over $x \in \Sigma$ is

$$
\left.B\right|_{x}=\left\{\left.u \in f^{*}\left(F_{\mathrm{G}_{2}}\right)\right|_{x}: u\left(T_{x} \Sigma\right)=\mathrm{A}^{\sharp} \oplus 0\right\}
$$

We recall (Proposition 2.6) that $\mathrm{G}_{2}$ acts transitively on the set of associative 3-planes with stabilizer $\mathrm{SO}(4)$, so $B \rightarrow \Sigma$ is a well-defined $\mathrm{SO}(4)$-bundle. Note that on $B$, we have

$$
\omega_{\mathrm{C}}=0 .
$$

For the rest of $\S 2.4$, all of our calculations will be done on the subbundle $B \subset F_{\mathrm{G}_{2}}$.

We now exploit splitting $T_{x} M=T_{x} \Sigma \oplus\left(T_{x} \Sigma\right)^{\perp} \simeq \mathrm{A}^{\sharp} \oplus \mathrm{C}^{\sharp}$ to decompose $\theta$ and $\gamma$ into $\mathrm{SO}(4)$ irreducible pieces. To decompose the connection 1 -form $\theta \in \Omega^{1}\left(B ; \mathfrak{g}_{2}\right)$, we split

$$
\mathfrak{g}_{2} \cong\left[\mathfrak{g}_{2} \cap\left(\Lambda^{2}(\mathrm{~A}) \oplus \Lambda_{+}^{2}(\mathrm{C})\right)\right] \oplus\left[\mathfrak{g}_{2} \cap(\mathrm{A} \otimes \mathrm{C})\right] \oplus\left[\mathfrak{g}_{2} \cap \Lambda_{-}^{2}(\mathrm{C})\right],
$$

so that $\theta$ takes the block form

$$
\theta=\left[\begin{array}{cc}
\rho(\zeta) & -\sigma^{T} \\
\sigma & \zeta+\xi
\end{array}\right]=\left[\begin{array}{ccc|cccc}
0 & 2 \zeta_{3} & -2 \zeta_{2} & -\sigma_{4}-\sigma_{7} & -\sigma_{3}+\sigma_{8} & \sigma_{2}+\sigma_{5} & \sigma_{1}-\sigma_{6} \\
-2 \zeta_{3} & 0 & 2 \zeta_{1} & \sigma_{1} & \sigma_{2} & \sigma_{3} & \sigma_{4} \\
2 \zeta_{2} & -2 \zeta_{1} & 0 & \sigma_{5} & \sigma_{6} & \sigma_{7} & \sigma_{8} \\
\hline \sigma_{4}+\sigma_{7} & -\sigma_{1} & -\sigma_{5} & 0 & -\zeta_{1}-\xi_{1} & -\zeta_{2}+\xi_{2} & \zeta_{3}+\xi_{3} \\
\sigma_{3}-\sigma_{8} & -\sigma_{2} & -\sigma_{6} & \zeta_{1}+\xi_{1} & 0 & \zeta_{3}-\xi_{3} & \zeta_{2}+\xi_{2} \\
-\sigma_{2}-\sigma_{5} & -\sigma_{3} & -\sigma_{7} & \zeta_{2}-\xi_{2} & -\zeta_{3}+\xi_{3} & 0 & -\zeta_{1}+\xi_{1} \\
-\sigma_{1}+\sigma_{6} & -\sigma_{4} & -\sigma_{8} & -\zeta_{3}-\xi_{3} & -\zeta_{2}-\xi_{2} & \zeta_{1}-\xi_{1} & 0
\end{array}\right] .
$$

Similarly, the 1 -form $\gamma \in \Omega^{1}\left(B ; \mathbb{R}^{7}\right)$ breaks into block form as:

$$
\gamma=\left[\begin{array}{cc}
\gamma_{\mathrm{A}} & -\left(\gamma_{\mathrm{C}}\right)^{T} \\
\gamma_{\mathrm{C}} & \left(\gamma_{\mathrm{A}}\right)_{+}
\end{array}\right]=\left[\begin{array}{ccc|cccc}
0 & \gamma_{3} & -\gamma_{2} & \gamma_{5} & -\gamma_{4} & \gamma_{7} & -\gamma_{6} \\
-\gamma_{3} & 0 & \gamma_{1} & \gamma_{6} & -\gamma_{7} & -\gamma_{4} & \gamma_{5} \\
\gamma_{2} & -\gamma_{1} & 0 & -\gamma_{7} & -\gamma_{6} & \gamma_{5} & \gamma_{4} \\
\hline-\gamma_{5} & -\gamma_{6} & \gamma_{7} & 0 & \gamma_{1} & \gamma_{2} & -\gamma_{3} \\
\gamma_{4} & \gamma_{7} & \gamma_{6} & -\gamma_{1} & 0 & -\gamma_{3} & -\gamma_{2} \\
-\gamma_{7} & \gamma_{4} & -\gamma_{5} & -\gamma_{2} & \gamma_{3} & 0 & \gamma_{1} \\
\gamma_{6} & -\gamma_{5} & -\gamma_{4} & \gamma_{3} & \gamma_{2} & -\gamma_{1} & 0
\end{array}\right]
$$

In this notation, the first structure equation (2.22) on $B$ reads:

$$
d\left(\begin{array}{c}
\omega_{\mathrm{A}} \\
0
\end{array}\right)=-\left(\left[\begin{array}{cc}
\rho(\zeta) & -\sigma^{T} \\
\sigma & \zeta+\xi
\end{array}\right]+2\left[\begin{array}{cc}
\gamma_{\mathrm{A}} & -\left(\gamma_{\mathrm{C}}\right)^{T} \\
\gamma_{\mathrm{C}} & \left(\gamma_{\mathrm{A}}\right)_{+}
\end{array}\right]\right) \wedge\left(\begin{array}{c}
\omega_{\mathrm{A}} \\
0
\end{array}\right)
$$


In particular, the second line gives

$$
0=-\left(\sigma+2 \gamma^{\mathrm{C}}\right) \wedge \omega_{\mathrm{A}}
$$

or in detail,

$$
\left[\begin{array}{ccc}
\sigma_{4}+\sigma_{7} & -\sigma_{1} & -\sigma_{5} \\
\sigma_{3}-\sigma_{8} & -\sigma_{2} & -\sigma_{6} \\
-\sigma_{2}-\sigma_{5} & -\sigma_{3} & -\sigma_{7} \\
-\sigma_{1}+\sigma_{6} & -\sigma_{4} & -\sigma_{8}
\end{array}\right] \wedge\left[\begin{array}{c}
\omega^{1} \\
\omega^{2} \\
\omega^{3}
\end{array}\right]=-2\left[\begin{array}{ccc}
-\gamma_{5} & -\gamma_{6} & \gamma_{7} \\
\gamma_{4} & \gamma_{7} & \gamma_{6} \\
-\gamma_{7} & \gamma_{4} & -\gamma_{5} \\
\gamma_{6} & -\gamma_{5} & -\gamma_{4}
\end{array}\right] \wedge\left[\begin{array}{c}
\omega^{1} \\
\omega^{2} \\
\omega^{3}
\end{array}\right]
$$

Note that on $B$, the 1 -forms $\sigma_{\delta}$ and $\gamma_{\alpha}$ are semibasic, and we write

$$
\sigma_{\delta}=S_{\delta p} \omega^{p} \quad \gamma_{\alpha}=T_{\alpha p} \omega^{p}
$$

for some function $S=\left(S_{\delta p}\right): B \rightarrow \mathrm{V}_{1,3} \otimes \mathrm{A}$, recalling our index ranges $1 \leq p \leq 3$ and $4 \leq \alpha \leq 7$ and $1 \leq \delta \leq 8$.

Now, the 24 functions $S_{\delta p}$ and the 12 functions $T_{\alpha p}$ are not independent: the equation (2.26) amounts to $12=4\left(\begin{array}{l}3 \\ 2\end{array}\right)$ linear relations among them. Explicitly:

$$
\left[\begin{array}{ccc}
S_{13}-S_{52} & S_{43}+S_{73}+S_{51} & -S_{42}-S_{72}-S_{11} \\
S_{23}-S_{62} & S_{33}-S_{83}+S_{61} & -S_{32}+S_{82}-S_{21} \\
S_{33}-S_{72} & -S_{23}-S_{53}+S_{71} & S_{22}+S_{52}-S_{31} \\
S_{43}-S_{82} & -S_{13}+S_{63}+S_{81} & S_{12}-S_{62}-S_{41}
\end{array}\right]=-2\left[\begin{array}{ccc}
T_{63}+T_{72} & -T_{53}-T_{71} & T_{52}-T_{61} \\
T_{62}-T_{73} & T_{43}-T_{61} & -T_{42}+T_{71} \\
-T_{43}-T_{52} & T_{51}-T_{73} & T_{41}+T_{72} \\
-T_{42}+T_{53} & T_{41}+T_{63} & -T_{51}-T_{62}
\end{array}\right]
$$

In particular, these relations imply:

$$
\begin{aligned}
S_{41}+S_{71}-S_{12}-S_{53} & =-4 \epsilon_{4 \alpha p} T_{\alpha p} \\
S_{31}-S_{81}-S_{22}-S_{63} & =-4 \epsilon_{5 \alpha p} T_{\alpha p} \\
-S_{21}-S_{51}-S_{32}-S_{73} & =-4 \epsilon_{6 \alpha p} T_{\alpha p} \\
-S_{11}+S_{61}-S_{42}-S_{83} & =-4 \epsilon_{7 \alpha p} T_{\alpha p}
\end{aligned}
$$

With these calculations in place, we may finally compute the mean curvature of an associative 3-fold:

Theorem 2.18. Let $\Sigma \subset M$ be an associative 3-fold immersed in a 7-manifold $M$ equipped with a $G_{2}$-structure. Then the mean curvature vector $H$ of $\Sigma$ is given by

$$
H=-3\left[\left(\tau_{1}\right) \mathrm{C}\right]^{\sharp}-\frac{\sqrt{3}}{2}\left[\left(\tau_{3}\right) \mathrm{c}\right]^{\ddagger} .
$$

In particular, the largest torsion class of $G_{2}$-structures $\varphi$ for which every associative 3 -fold is minimal is $W_{1} \oplus W_{14}=W_{1} \cup W_{14}$, i.e., the class for which $d \varphi=\lambda * \varphi$ for some $\lambda \in \mathbb{R}$.

Proof. The mean curvature vector may be computed as follows:

$$
\begin{aligned}
& {\left[\begin{array}{l}
H_{4} \\
H_{5} \\
H_{6} \\
H_{7}
\end{array}\right] \omega^{123}=\left[\begin{array}{lll}
\psi_{41} & \psi_{42} & \psi_{43} \\
\psi_{51} & \psi_{52} & \psi_{53} \\
\psi_{61} & \psi_{62} & \psi_{63} \\
\psi_{71} & \psi_{72} & \psi_{73}
\end{array}\right] \wedge\left[\begin{array}{l}
\omega^{23} \\
\omega^{31} \\
\omega^{12}
\end{array}\right] } \\
&= {\left[\begin{array}{ccc}
\sigma_{4}+\sigma_{7} & -\sigma_{1} & -\sigma_{5} \\
\sigma_{3}-\sigma_{8} & -\sigma_{2} & -\sigma_{6} \\
-\sigma_{2}-\sigma_{5} & -\sigma_{3} & -\sigma_{7} \\
-\sigma_{1}+\sigma_{6} & -\sigma_{4} & -\sigma_{8}
\end{array}\right] \wedge\left[\begin{array}{l}
\omega^{23} \\
\omega^{31} \\
\omega^{12}
\end{array}\right]+2\left[\begin{array}{ccc}
-\gamma_{5} & -\gamma_{6} & \gamma_{7} \\
\gamma_{4} & \gamma_{7} & \gamma_{6} \\
-\gamma_{7} & \gamma_{4} & -\gamma_{5} \\
\gamma_{6} & -\gamma_{5} & -\gamma_{4}
\end{array}\right] \wedge\left[\begin{array}{c}
\omega^{23} \\
\omega^{31} \\
\omega^{12}
\end{array}\right] }
\end{aligned}
$$


To evaluate the first term in (2.28), we substitute $\sigma_{\delta}=S_{\delta p} \omega^{p}$, followed by (2.27), and finally (2.23), to obtain:

$$
\begin{aligned}
& {\left[\begin{array}{ccc}
\sigma_{4}+\sigma_{7} & -\sigma_{1} & -\sigma_{5} \\
\sigma_{3}-\sigma_{8} & -\sigma_{2} & -\sigma_{6} \\
-\sigma_{2}-\sigma_{5} & -\sigma_{3} & -\sigma_{7} \\
-\sigma_{1}+\sigma_{6} & -\sigma_{4} & -\sigma_{8}
\end{array}\right] \wedge\left[\begin{array}{l}
\omega^{23} \\
\omega^{31} \\
\omega^{12}
\end{array}\right]=} {\left[\begin{array}{c}
S_{41}+S_{71}-S_{12}-S_{53} \\
S_{31}-S_{81}-S_{22}-S_{63} \\
-S_{21}-S_{51}-S_{32}-S_{73} \\
-S_{11}+S_{61}-S_{42}-S_{83}
\end{array}\right] \omega^{123} } \\
&=-4\left[\begin{array}{l}
\epsilon_{4 \alpha p} T_{\alpha p} \\
\epsilon_{5 \alpha p} T_{\alpha p} \\
\epsilon_{6 \alpha p} T_{\alpha p} \\
\epsilon_{7 \alpha p} T_{\alpha p}
\end{array}\right] \omega^{123}=-12\left[\begin{array}{l}
B_{4}+M_{4} \\
B_{5}+M_{5} \\
B_{6}+M_{6} \\
B_{7}+M_{7}
\end{array}\right] \omega^{123}
\end{aligned}
$$

Similarly, to evaluate the second term in (2.28), we substitute $\gamma_{\alpha}=T_{\alpha p} \omega^{p}$ followed by (2.23) to obtain:

$$
2\left[\begin{array}{ccc}
-\gamma_{5} & -\gamma_{6} & \gamma_{7} \\
\gamma_{4} & \gamma_{7} & \gamma_{6} \\
-\gamma_{7} & \gamma_{4} & -\gamma_{5} \\
\gamma_{6} & -\gamma_{5} & -\gamma_{4}
\end{array}\right] \wedge\left[\begin{array}{c}
\omega^{23} \\
\omega^{31} \\
\omega^{12}
\end{array}\right]=-2\left[\begin{array}{c}
\epsilon_{4 \alpha p} T_{\alpha p} \\
\epsilon_{5 \alpha p} T_{\alpha p} \\
\epsilon_{6 \alpha p} T_{\alpha p} \\
\epsilon_{7 \alpha p} T_{\alpha p}
\end{array}\right] \omega^{123}=-6\left[\begin{array}{c}
B_{4}+M_{4} \\
B_{5}+M_{5} \\
B_{6}+M_{6} \\
B_{7}+M_{7}
\end{array}\right] \omega^{123}
$$

We conclude that

$$
H_{\alpha}=-18 B_{\alpha}-18 M_{\alpha}
$$

and so (2.21) yields

$$
H=-3\left[\left(\tau_{1}\right) \mathrm{c}\right]^{\sharp}-\frac{\sqrt{3}}{2}\left[\left(\tau_{3}\right) \mathrm{C}\right]^{\ddagger} .
$$

In particular, the largest torsion class for which $H=0$ for all associatives is the one for which $\tau_{1}=\tau_{3}=0$, which is $W_{1} \oplus W_{14}=W_{1} \cup W_{14}$.

\subsection{Mean Curvature of Coassociative 4-Folds}

In this subsection, we derive a formula (Theorem 2.21) for the mean curvature of a coassociative 4-fold in an arbitrary 7 -manifold $(M, \varphi)$ with $\mathrm{G}_{2}$-structure $\varphi$. In the process, we observe a necessary condition (Theorem 2.19) for the local existence of coassociative 4-folds. We continue with the notation of $\S 2.3$.

Let $f: \Sigma^{4} \rightarrow M^{7}$ denote an immersion of a coassociative 4 -fold into $M$, and let $f^{*}\left(F_{\mathrm{G}_{2}}\right) \rightarrow \Sigma$ denote the pullback bundle. Let $B \subset f^{*}\left(F_{\mathrm{G}_{2}}\right)$ denote the subbundle of coframes adapted to $\Sigma$, i.e., the subbundle whose fiber over $x \in \Sigma$ is

$$
\left.B\right|_{x}=\left\{\left.u \in f^{*}\left(F_{\mathrm{G}_{2}}\right)\right|_{x}: u\left(T_{x} \Sigma\right)=0 \oplus \mathrm{C}^{\sharp}\right\}
$$

We recall (Proposition 2.6) that $\mathrm{G}_{2}$ acts transitively on the set of coassociative 4-planes with stabilizer $\mathrm{SO}(4)$, so $B \rightarrow \Sigma$ is a well-defined $\mathrm{SO}(4)$-bundle. Note that on $B$, we have

$$
\omega_{\mathrm{A}}=0 .
$$

For the rest of $\S 2.5$, all of our calculations will be done on the subbundle $B \subset F_{\mathrm{G}_{2}}$. 
As in $\S 2.4$, we use the splitting $T_{x} M=\left(T_{x} \Sigma\right)^{\perp} \oplus T_{x} \Sigma \simeq \mathrm{A}^{\sharp} \oplus \mathrm{C}^{\sharp}$ to decompose $\theta$ and $\gamma$ into $\mathrm{SO}(4)$-irreducible pieces. The result is the identical: the connection 1-form $\theta \in \Omega^{1}\left(B ; \mathfrak{g}_{2}\right)$ takes the block form

$$
\theta=\left[\begin{array}{cc}
\rho(\zeta) & -\sigma^{T} \\
\sigma & \zeta+\xi
\end{array}\right]=\left[\begin{array}{ccc|cccc}
0 & 2 \zeta_{3} & -2 \zeta_{2} & -\sigma_{4}-\sigma_{7} & -\sigma_{3}+\sigma_{8} & \sigma_{2}+\sigma_{5} & \sigma_{1}-\sigma_{6} \\
-2 \zeta_{3} & 0 & 2 \zeta_{1} & \sigma_{1} & \sigma_{2} & \sigma_{3} & \sigma_{4} \\
2 \zeta_{2} & -2 \zeta_{1} & 0 & \sigma_{5} & \sigma_{6} & \sigma_{7} & \sigma_{8} \\
\hline \sigma_{4}+\sigma_{7} & -\sigma_{1} & -\sigma_{5} & 0 & -\zeta_{1}-\xi_{1} & -\zeta_{2}+\xi_{2} & \zeta_{3}+\xi_{3} \\
\sigma_{3}-\sigma_{8} & -\sigma_{2} & -\sigma_{6} & \zeta_{1}+\xi_{1} & 0 & \zeta_{3}-\xi_{3} & \zeta_{2}+\xi_{2} \\
-\sigma_{2}-\sigma_{5} & -\sigma_{3} & -\sigma_{7} & \zeta_{2}-\xi_{2} & -\zeta_{3}+\xi_{3} & 0 & -\zeta_{1}+\xi_{1} \\
-\sigma_{1}+\sigma_{6} & -\sigma_{4} & -\sigma_{8} & -\zeta_{3}-\xi_{3} & -\zeta_{2}-\xi_{2} & \zeta_{1}-\xi_{1} & 0
\end{array}\right] .
$$

and the 1-form $\gamma \in \Omega^{1}\left(B ; \mathbb{R}^{7}\right)$ takes the block form

$$
\gamma=\left[\begin{array}{cc}
\gamma_{\mathrm{A}} & -\left(\gamma_{\mathrm{C}}\right)^{T} \\
\gamma_{\mathrm{C}} & \left(\gamma_{\mathrm{A}}\right)_{+}
\end{array}\right]=\left[\begin{array}{ccc|cccc}
0 & \gamma_{3} & -\gamma_{2} & \gamma_{5} & -\gamma_{4} & \gamma_{7} & -\gamma_{6} \\
-\gamma_{3} & 0 & \gamma_{1} & \gamma_{6} & -\gamma_{7} & -\gamma_{4} & \gamma_{5} \\
\gamma_{2} & -\gamma_{1} & 0 & -\gamma_{7} & -\gamma_{6} & \gamma_{5} & \gamma_{4} \\
\hline-\gamma_{5} & -\gamma_{6} & \gamma_{7} & 0 & \gamma_{1} & \gamma_{2} & -\gamma_{3} \\
\gamma_{4} & \gamma_{7} & \gamma_{6} & -\gamma_{1} & 0 & -\gamma_{3} & -\gamma_{2} \\
-\gamma_{7} & \gamma_{4} & -\gamma_{5} & -\gamma_{2} & \gamma_{3} & 0 & \gamma_{1} \\
\gamma_{6} & -\gamma_{5} & -\gamma_{4} & \gamma_{3} & \gamma_{2} & -\gamma_{1} & 0
\end{array}\right]
$$

In this notation, the first structure equation $(2.22)$ on $B$ reads:

$$
d\left(\begin{array}{c}
0 \\
\omega_{\mathrm{C}}
\end{array}\right)=-\left(\left[\begin{array}{cc}
\rho(\zeta) & -\sigma^{T} \\
\sigma & \zeta+\xi
\end{array}\right]+2\left[\begin{array}{cc}
\gamma_{\mathrm{A}} & -\left(\gamma_{\mathrm{C}}\right)^{T} \\
\gamma_{\mathrm{C}} & \left(\gamma_{\mathrm{A}}\right)_{+}
\end{array}\right]\right) \wedge\left(\begin{array}{c}
0 \\
\omega_{\mathrm{C}}
\end{array}\right)
$$

In particular, the first line gives

$$
0=\left(\sigma^{T}+2\left(\gamma_{\mathrm{C}}\right)^{T}\right) \wedge \omega_{\mathrm{C}}
$$

or in full detail,

$$
\left[\begin{array}{cccc}
-\sigma_{4}-\sigma_{7} & -\sigma_{3}+\sigma_{8} & \sigma_{2}+\sigma_{5} & \sigma_{1}-\sigma_{6} \\
\sigma_{1} & \sigma_{2} & \sigma_{3} & \sigma_{4} \\
\sigma_{5} & \sigma_{6} & \sigma_{7} & \sigma_{8}
\end{array}\right] \wedge\left[\begin{array}{c}
\eta^{1} \\
\eta^{2} \\
\eta^{3} \\
\eta^{4}
\end{array}\right]=-2\left[\begin{array}{cccc}
\gamma_{5} & -\gamma_{4} & \gamma_{7} & -\gamma_{6} \\
\gamma_{6} & -\gamma_{7} & -\gamma_{4} & \gamma_{5} \\
-\gamma_{7} & -\gamma_{6} & \gamma_{5} & \gamma_{4}
\end{array}\right] \wedge\left[\begin{array}{c}
\eta^{1} \\
\eta^{2} \\
\eta^{3} \\
\eta^{4}
\end{array}\right]
$$

Note that on $B$, the 1 -forms $\sigma_{\delta}$ and $\gamma_{\alpha}$ are semibasic, so we can write

$$
\sigma_{\delta}=S_{\delta \alpha} \omega^{\alpha} \quad \gamma_{\beta}=T_{\beta \alpha} \omega^{\alpha}
$$

for some function $S=\left(S_{\delta \alpha}\right): B \rightarrow \mathrm{V}_{1,3} \otimes \mathrm{C}$, recalling our index ranges $1 \leq p \leq 3$ and $4 \leq \alpha, \beta \leq 7$ and $1 \leq \delta \leq 8$.

Note that the 32 functions $S_{\delta \alpha}$ and the 16 functions $T_{\beta \alpha}$ are not independent: the equation (2.29) shows that they satisfy $3\left(\begin{array}{l}4 \\ 2\end{array}\right)=18$ linear relations. Explicitly:

$$
\left[\begin{array}{ccc}
S_{15}-S_{24} & S_{55}-S_{64} & S_{84}+S_{45}+S_{75}-S_{34} \\
S_{16}-S_{34} & S_{56}-S_{74} & S_{54}+S_{24}+S_{46}+S_{76} \\
S_{26}-S_{35} & S_{66}-S_{75} & S_{14}-S_{64}+S_{47}+S_{77} \\
S_{17}-S_{44} & S_{57}-S_{84} & S_{55}-S_{86}+S_{25}+S_{36} \\
S_{27}-S_{45} & S_{67}-S_{85} & -S_{65}+S_{15}+S_{37}-S_{87} \\
S_{37}-S_{46} & S_{77}-S_{86} & -S_{66}-S_{57}+S_{16}-S_{27}
\end{array}\right]=2\left[\begin{array}{ccc}
-T_{74}-T_{65} & -T_{64}+T_{75} & T_{44}+T_{55} \\
-T_{44}-T_{66} & T_{54}+T_{76} & -T_{74}+T_{56} \\
-T_{45}+T_{76} & T_{55}+T_{66} & T_{64}+T_{57} \\
T_{54}-T_{67} & T_{44}+T_{77} & -T_{75}-T_{46} \\
T_{55}+T_{77} & T_{45}+T_{67} & T_{65}-T_{47} \\
T_{56}+T_{47} & T_{46}-T_{57} & T_{66}+T_{77}
\end{array}\right]
$$


We make two observations on this system of equations. First, we observe the relation

$$
\left(T_{44}+T_{55}\right)+\left(T_{55}+T_{66}\right)+\left(T_{55}+T_{77}\right)+\left(T_{66}+T_{77}\right)+\left(T_{44}+T_{77}\right)+\left(T_{44}+T_{66}\right)=0 .
$$

Substituting (2.24), this equation simplifies to

$$
3 F+\frac{1}{24} \tau_{0}=0
$$

Substituting (2.21), we have proved:

Theorem 2.19. If a coassociative 4-fold $\Sigma$ exists in $M$, then the following relation holds at points of $\Sigma$ :

$$
\tau_{0}=-\frac{\sqrt{42}}{7}\left[\left(\tau_{3}\right)_{0,0}\right]^{\dagger}
$$

In particular, if $\tau_{3}=0$ and $\tau_{0}$ is non-vanishing (so the torsion takes values in $\left(W_{1} \oplus W_{7} \oplus W_{14}\right)$ $\left.\left(W_{7} \oplus W_{14}\right)\right)$, then $M$ admits no coassociative 4-folds (even locally).

Corollary 2.20. Fix $x \in M$. If every coassociative 4-plane in $T_{x} M$ is tangent to a coassociative 4-fold, then $\left.\tau_{0}\right|_{x}=0$ and $\left.\tau_{3}\right|_{x}=0$.

Proof. The hypotheses imply that equation (2.30) holds for all coassociative 4-planes at $x \in M$. Thus, we have a $\mathrm{G}_{2}$-invariant linear relation between $\left.\tau_{0}\right|_{x}$ and $\left.\tau_{3}\right|_{x}$. This implies that $\left.\tau_{0}\right|_{x}=0$ and $\left.\tau_{3}\right|_{x}=0$ by Schur's Lemma.

Second, we observe the three relations

$$
\begin{aligned}
\left(S_{17}-S_{67}\right)+\left(S_{26}+S_{56}\right)+\left(-S_{35}+S_{85}\right)-\left(S_{44}+S_{74}\right) & =-4\left(T_{45}-T_{54}\right)-4\left(T_{67}-T_{76}\right) \\
S_{14}+S_{25}+S_{36}+S_{47} & =4\left(T_{57}-T_{75}\right)-4\left(T_{46}-T_{64}\right) \\
S_{54}+S_{65}+S_{76}+S_{87} & =4\left(T_{47}-T_{74}\right)+4\left(T_{56}-T_{65}\right) .
\end{aligned}
$$

We may now compute the mean curvature of a coassociative 4-fold:

Theorem 2.21. Let $\Sigma \subset M$ be a coassociative 4-fold immersed in a 7-manifold $M$ equipped with a $G_{2}$-structure. Then the mean curvature vector $H$ of $\Sigma$ is given by

$$
H=-4\left[\left(\tau_{1}\right)_{\mathrm{A}}\right]^{\sharp}+\frac{\sqrt{6}}{3}\left[\left(\tau_{2}\right)_{\mathrm{A}}\right]^{\natural} .
$$

In particular, the largest torsion class of $G_{2}$-structures $\varphi$ for which every coassociative 4-fold is minimal is $W_{1} \oplus W_{27}$, i.e., the class for which $d * \varphi=0$.

Proof. Let $\beta_{\alpha}:=*_{\mathrm{C}}\left(\omega^{\alpha}\right) \in \Omega^{3}(B)$ and $\operatorname{vol}_{\mathrm{C}}=\omega^{4567}$. The mean curvature vector may be computed 
as follows:

$$
\begin{aligned}
{\left[\begin{array}{l}
H^{1} \\
H^{2} \\
H^{3}
\end{array}\right] \operatorname{vol}_{\mathrm{C}} } & =\left[\begin{array}{llll}
\psi_{14} & \psi_{15} & \psi_{16} & \psi_{17} \\
\psi_{24} & \psi_{25} & \psi_{26} & \psi_{27} \\
\psi_{34} & \psi_{35} & \psi_{36} & \psi_{37}
\end{array}\right] \wedge\left[\begin{array}{c}
\beta_{4} \\
\beta_{5} \\
\beta_{6} \\
\beta_{7}
\end{array}\right] \\
& =\left[\begin{array}{cccc}
-\sigma_{4}-\sigma_{7} & -\sigma_{3}+\sigma_{8} & \sigma_{2}+\sigma_{5} & \sigma_{1}-\sigma_{6} \\
\sigma_{1} & \sigma_{2} & \sigma_{3} & \sigma_{4} \\
\sigma_{5} & \sigma_{6} & \sigma_{7} & \sigma_{8}
\end{array}\right] \wedge\left[\begin{array}{c}
\beta_{4} \\
\beta_{5} \\
\beta_{6} \\
\beta_{7}
\end{array}\right]+2\left[\begin{array}{cccc}
\gamma_{5} & -\gamma_{4} & \gamma_{7} & -\gamma_{6} \\
\gamma_{6} & -\gamma_{7} & -\gamma_{4} & \gamma_{5} \\
-\gamma_{7} & -\gamma_{6} & \gamma_{5} & \gamma_{4}
\end{array}\right] \wedge\left[\begin{array}{c}
\beta_{4} \\
\beta_{5} \\
\beta_{6} \\
\beta_{7}
\end{array}\right]
\end{aligned}
$$

To evaluate the first term in (2.32), we substitute $\sigma_{\delta}=S_{\delta p} \omega^{p}$, followed by (2.31), and finally (2.25), to obtain:

$$
\begin{gathered}
{\left[\begin{array}{cccc}
-\sigma_{4}-\sigma_{7} & -\sigma_{3}+\sigma_{8} & \sigma_{2}+\sigma_{5} & \sigma_{1}-\sigma_{6} \\
\sigma_{1} & \sigma_{2} & \sigma_{3} & \sigma_{4} \\
\sigma_{5} & \sigma_{6} & \sigma_{7} & \sigma_{8}
\end{array}\right] \wedge\left[\begin{array}{c}
\beta_{4} \\
\beta_{5} \\
\beta_{6} \\
\beta_{7}
\end{array}\right]=4\left[\begin{array}{c}
-\left(T_{45}-T_{54}\right)-\left(T_{67}-T_{76}\right) \\
\left(T_{57}-T_{75}\right)-\left(T_{46}-T_{64}\right) \\
\left(T_{47}-T_{74}\right)+\left(T_{56}-T_{65}\right)
\end{array}\right] \operatorname{vol}_{\mathrm{C}}} \\
=16\left[\begin{array}{l}
-A_{1}+C_{1} \\
-A_{2}+C_{2} \\
-A_{3}+C_{3}
\end{array}\right] \operatorname{vol}_{\mathrm{C}}
\end{gathered}
$$

Similarly, to evaluate the second term in (2.32), we substitute $\gamma_{\alpha}=T_{\alpha p} \omega^{p}$ followed by (2.25) to obtain:

$$
\begin{aligned}
2\left[\begin{array}{cccc}
\gamma_{5} & -\gamma_{4} & \gamma_{7} & -\gamma_{6} \\
\gamma_{6} & -\gamma_{7} & -\gamma_{4} & \gamma_{5} \\
-\gamma_{7} & -\gamma_{6} & \gamma_{5} & \gamma_{4}
\end{array}\right] \wedge\left[\begin{array}{l}
\beta_{4} \\
\beta_{5} \\
\beta_{6} \\
\beta_{7}
\end{array}\right]=2\left[\begin{array}{c}
-\left(T_{45}-T_{54}\right)-\left(T_{67}-T_{76}\right) \\
\left(T_{57}-T_{75}\right)-\left(T_{46}-T_{64}\right) \\
\left(T_{47}-T_{74}\right)+\left(T_{56}-T_{65}\right)
\end{array}\right] \operatorname{vol}_{\mathrm{C}} \\
=8\left[\begin{array}{l}
-A_{1}+C_{1} \\
-A_{2}+C_{2} \\
-A_{3}+C_{3}
\end{array}\right] \operatorname{vol}_{\mathrm{C}}
\end{aligned}
$$

We conclude that

$$
H_{p}=-24 A_{p}+24 C_{p}
$$

and so (2.21) yields

$$
H=-4\left[\left(\tau_{1}\right)_{\mathrm{A}}\right]^{\sharp}+\frac{\sqrt{6}}{3}\left[\left(\tau_{2}\right)_{\mathrm{A}}\right]^{\natural} .
$$

In particular, the largest torsion class for which $H=0$ for all coassociatives is the one for which $\tau_{1}=\tau_{2}=0$, which is $W_{1} \oplus W_{27}$.

\section{Cayley 4-Folds in Spin(7)-Structures}

\subsection{Preliminaries}

In this subsection, we define both the ambient spaces and the submanifolds of interest. This subsection will also be used to fix notation and conventions. 


\subsubsection{Spin(7)-Structures on Vector Spaces}

Let $V=\mathbb{R}^{8}$ equipped with the standard inner product $\langle\cdot, \cdot\rangle$, norm $\|\cdot\|$, and an orientation. Let $\left\{e_{1}, \ldots, e_{8}\right\}$ denote the standard (orthonormal) basis of $V$, and let $\left\{e^{1}, \ldots, e^{8}\right\}$ denote the corresponding dual basis of $V^{*}$. The Cayley 4-form is the alternating 4-form $\Phi_{0} \in \Lambda^{4}\left(V^{*}\right)$ defined by

$\Phi_{0}=e^{1234}+\left(e^{12}+e^{34}\right) \wedge\left(e^{56}+e^{78}\right)+\left(e^{13}-e^{24}\right) \wedge\left(e^{57}-e^{68}\right)+\left(-e^{14}-e^{23}\right) \wedge\left(e^{58}+e^{67}\right)+e^{5678}$.

For calculations, it will be convenient to express $\Phi_{0}$ in the form

$$
\Phi_{0}=\frac{1}{24} \Phi_{i j k l} e^{i j k l},
$$

where the constants $\Phi_{i j k l} \in\{-1,0,1\}$ are defined by this formula and skew-symmetry.

The stabilizer of $\Phi_{0}$ under the pullback action of $G L(V)$ is isomorphic to $\operatorname{Spin}(7)$,

$$
\operatorname{Spin}(7) \cong\left\{A \in \mathrm{GL}(V): A^{*} \Phi_{0}=\Phi_{0}\right\} .
$$

Using the isomorphism (3.1), we regard $\operatorname{Spin}(7)$ as a subgroup of $G L(V)$. As a $\operatorname{Spin}(7)$-module, $V$ is isomorphic to the spinor representation of $\operatorname{Spin}(7)$, and is thus irreducible. The induced representation on $\Lambda^{k}\left(V^{*}\right)$ for $2 \leq k \leq 6$ is reducible, and there are irreducible decompositions [4]

$$
\begin{aligned}
& \Lambda^{2}\left(V^{*}\right) \cong \Lambda_{7}^{2} \oplus \Lambda_{21}^{2}, \\
& \Lambda^{3}\left(V^{*}\right) \cong \Lambda_{8}^{3} \oplus \Lambda_{48}^{3}, \\
& \Lambda^{4}\left(V^{*}\right) \cong \Lambda_{1}^{4} \oplus \Lambda_{7}^{4} \oplus \Lambda_{27}^{4} \oplus \Lambda_{35}^{4} .
\end{aligned}
$$

We will need the definitions of the irreducible pieces of $\Lambda^{2}\left(V^{*}\right)$ and $\Lambda^{3}\left(V^{*}\right)$, they are

$$
\begin{aligned}
\Lambda_{7}^{2} & =\left\{\beta \in \Lambda^{2}\left(V^{*}\right) \mid *\left(\Phi_{0} \wedge \beta\right)=3 \beta\right\}, \\
\Lambda_{21}^{2} & =\left\{\beta \in \Lambda^{2}\left(V^{*}\right) \mid *\left(\Phi_{0} \wedge \beta\right)=-\beta\right\} \cong \mathfrak{s p i n}(7), \\
\Lambda_{8}^{3} & =\left\{*\left(\Phi_{0} \wedge \alpha\right) \mid \alpha \in V^{*}\right\}, \\
\Lambda_{48}^{3} & =\left\{\gamma \in \Lambda^{3}\left(V^{*}\right) \mid \Phi_{0} \wedge \gamma=0\right\} .
\end{aligned}
$$

The irreducible decompositions of the spaces $\Lambda^{5}\left(V^{*}\right)$ and $\Lambda^{6}\left(V^{*}\right)$ can be obtained by applying the Hodge star operator to the decompositions of $\Lambda^{3}\left(V^{*}\right)$ and $\Lambda^{2}\left(V^{*}\right)$ respectively.

\subsection{2 $\operatorname{Spin}(7)-S t r u c t u r e s$ on 8 -Manifolds}

Let $M$ be an oriented 8-manifold. A Spin(7)-structure on $M$ is a differential 4-form $\Phi \in \Omega^{4}(M)$ such that at each $x \in M$, and after identifying $T_{x} M$ with $V=\mathbb{R}^{8}$, the 4-form $\left.\Phi\right|_{x} \in \Lambda^{4}\left(T_{x}^{*} M\right)$ lies in the $G L(V)$-orbit of $\Phi_{0}$. That is, at each $x \in M$ there exists a coframe $u: T_{x} M \rightarrow \mathbb{R}^{8}$ for which $\left.\Phi\right|_{x}=u^{*}\left(\Phi_{0}\right)$.

The first order local invariants of a Spin(7)-structure are completely encoded in a Spin(7)equivariant function

$$
T: F_{\mathrm{Spin}(7)} \rightarrow \Lambda^{1} \oplus \Lambda_{48}^{3}
$$

called the intrinsic torsion function, defined on the total space of the $\operatorname{Spin}(7)$-frame bundle $F_{\mathrm{Spin}(7)} \rightarrow$ $M$ over $M$. 
By a result of Fernández [6], the intrinsic torsion function of a $\operatorname{Spin}(7)$-structure is equivalent to the data of the 5 -form $d \Phi$. It follows from the decomposition of $\Lambda^{5}\left(V^{*}\right)$ described in the previous section that the exterior derivative of $\Phi$ takes the form

$$
d \Phi=\tau_{1} \wedge \Phi+* \tau_{3},
$$

where $\left(\tau_{1}, \tau_{3}\right) \in \Gamma\left(\Lambda^{1}\left(T^{*} M\right) \oplus \Lambda_{48}^{3}\left(T^{*} M\right)\right)$. We refer to the forms $\tau_{1}$ and $\tau_{3}$ as the torsion forms of the $\operatorname{Spin}(7)$-structure.

\subsubsection{Cayley 4-Folds}

Let $(M, \Phi)$ be an 8-manifold with a $\operatorname{Spin}(7)$-structure, and consider a tangent space $\left(T_{x} M,\left.\Phi\right|_{x}\right) \cong$ $\left(V, \Phi_{0}\right)$. The vector space $\left(V, \Phi_{0}\right)$ possesses a distinguished class of subspaces - the Cayley 4-planes — first studied by Harvey and Lawson [9] in their work on calibrations.

Proposition 3.1 ([9]). The Cayley 4-form $\Phi_{0}$ has co-mass one, meaning that

$$
\left|\Phi_{0}(x, y, z, w)\right| \leq 1
$$

for every orthonormal set $\{x, y, z, w\}$ in $V \cong \mathbb{R}^{7}$.

Proposition $3.2([9])$. Let $K \in G r_{4}(V)$ be a 4-plane in $V$. The following are equivalent

1. If $\{x, y, z, w\}$ is an orthonormal basis of $K$, then $\Phi_{0}(x, y, z, w)= \pm 1$.

2. $\operatorname{Im}(x \times y \times z \times w)=0$ for any basis $x, y, z, w$ of $K$.

3. $y \times z \times w \in K$ for all $y, z, w \in K$.

The $\operatorname{Spin}(7)$-action on $V$ induces $\operatorname{Spin}(7)$-actions on the $\operatorname{Grassmannians} \mathrm{Gr}_{k}(V)$ of $k$-planes in $V$. These actions are transitive for $k=1,2,3,5,6,7$, but the action is not transitive when $k=4$. Indeed, the (proper) subset consisting of Cayley 4-planes is a $\operatorname{Spin}(7)$-orbit.

Proposition 3.3. The Lie group Spin(7) acts transitively on the subset of Cayley 4-planes

$$
\left\{E \in G r_{4}(V):\left|\Phi_{0}(E)\right|=1\right\} \subset G r_{4}(V)
$$

with stabiliser isomorphic to $S U(2) \times S U(2) \times S U(2) /\{ \pm I d\}$.

Notation: We will denote the group $S U(2)^{3} /\{ \pm \operatorname{Id}\}$ by $\operatorname{Spin}^{h}(4)$.

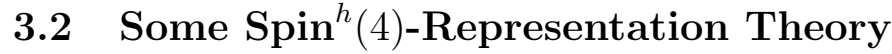

The Lie group $\operatorname{Spin}^{h}(4)$ is double-covered by the simply-connected group $\mathrm{SU}(2)^{3}$. The complex irreducible representations of $\mathrm{SU}(2)^{3}$ are exactly the tensor products $\mathrm{V}_{p} \otimes \mathrm{V}_{q} \otimes \mathrm{V}_{r}$ of irreducible $\mathrm{SU}(2)$-representations for each factor. The complex irreducible representations of $\mathrm{SU}(2)$ are well known to be the spaces of homogeneous polynomials in two variables of fixed degree, $\mathrm{V}_{p}=\operatorname{Sym}^{p}(\mathbb{C}\langle x, y\rangle)$.

Let $\mathrm{V}_{p, q, r}^{\mathbb{C}}$ denote $\mathrm{V}_{p} \otimes \mathrm{V}_{q} \otimes \mathrm{V}_{r}$. We think of $\mathrm{V}_{p, q, r}^{\mathbb{C}}$ as the space of homogeneous polynomials in $(u, v ; x, y ; w, z)$ of tridegree $(p, q, r)$. When $p+q+r$ is even the representation $\mathbb{V}_{p, q, r}^{\mathbb{C}}$ descends to a representation of $\operatorname{Spin}^{h}(4)$, and each of these representations has a real structure induced by the map $(u, v, x, y, w, z) \mapsto(v,-u, y,-x, z,-w)$. This yields a complete description of the real representations of $\operatorname{Spin}^{h}(4)$. We work with real representations, letting $\mathrm{V}_{p, q, r}$ denote the real representation underlying $\mathrm{V}_{p, q, r}^{\mathbb{C}}$. 
The Clebsch-Gordan formula applied to each SU(2) representation gives the irreducible decomposition of a tensor product of $\operatorname{Spin}^{h}(4)$-modules:

$$
\mathrm{V}_{p_{1}, q_{1}, r_{1}} \otimes \mathrm{V}_{p_{2}, q_{2}, r_{2}} \cong \bigoplus_{i=0}^{\left|p_{1}-p_{2}\right|} \bigoplus_{j=0}^{\left|q_{1}-q_{2}\right|} \bigoplus_{k=0}^{\left|r_{1}-r_{2}\right|} \mathrm{V}_{p_{1}+p_{2}-2 i, q_{1}+q_{2}-2 j, r_{1}+r_{2}-2 k}
$$

\subsection{1 $\operatorname{Spin}^{h}(4)$ as a subgroup of $\operatorname{Spin}(7)$}

In our calculations we shall need a concrete realization of $\operatorname{Spin}^{h}(4)$ as the stabilizer of a Cayley plane. Let $\operatorname{Spin}^{h}(4)$ act on $V \cong \mathbb{R}^{8}$ via the identification $V \cong \mathrm{V}_{1,1,0} \oplus \mathrm{V}_{0,1,1}$. Define a basis $\left(e_{1}, \ldots, e_{8}\right)$ of $V$ by

$$
\begin{array}{llll}
e_{1}=i(-u x+v y), & e_{2}=u x+v y, & e_{3}=-i(u y+v x), & e_{4}=-u y+v x, \\
e_{5}=i(x w-y z), & e_{6}=x w+y z, & e_{7}=-i(x z+y w), & e_{8}=-x z+y w .
\end{array}
$$

Then the 4-form

$$
e^{1234}+\left(e^{12}+e^{34}\right) \wedge\left(e^{56}+e^{78}\right)+\left(e^{13}-e^{24}\right) \wedge\left(e^{57}-e^{68}\right)+\left(-e^{14}-e^{23}\right) \wedge\left(e^{58}+e^{67}\right)+e^{5678}
$$

is $\operatorname{Spin}^{h}(4)$-invariant, and thus the action of $\operatorname{Spin}^{h}(4)$ on $V$ gives an embedding $\operatorname{Spin}^{h}(4) \subset \operatorname{Spin}(7)$. The 4-plane $\left\langle e_{1}, e_{2}, e_{3}, e_{4}\right\rangle$ is Cayley and is preserved by the action of $\operatorname{Spin}^{h}(4)$.

\subsubsection{Decomposition of 1-forms on $V$}

Let $V$ be as above. We have

$$
\Lambda^{1}\left(V^{*}\right)=\mathrm{K} \oplus \mathrm{L}
$$

where

$$
\begin{aligned}
\mathrm{K} & =\left\langle e_{1}, e_{2}, e_{3}, e_{4}\right\rangle, \\
\mathrm{L} & =\left\langle e_{5}, e_{6}, e_{7}, e_{8}\right\rangle .
\end{aligned}
$$

As abstract $\operatorname{Spin}^{h}(4)$-modules, $\mathrm{K} \cong \mathrm{V}_{1,1,0}$ and $\mathrm{L} \cong \mathrm{V}_{0,1,1}$.

\subsubsection{Decomposition of 2-forms on $V$}

We now seek to decompose $\Lambda^{2}\left(V^{*}\right)$ into $\operatorname{Spin}^{h}(4)$-irreducible submodules. As noted in $\S 3.1$ above, $\Lambda^{2}\left(V^{*}\right)$ splits into $\operatorname{Spin}(7)$-irreducible submodules as

$$
\Lambda^{2}\left(V^{*}\right) \cong \Lambda_{7}^{2} \oplus \Lambda_{21}^{2}
$$

On the other hand, using $V^{*} \cong \mathrm{K} \oplus \mathrm{L}$, we may also decompose $\Lambda^{2}\left(V^{*}\right)$ as

$$
\Lambda^{2}\left(V^{*}\right) \cong \Lambda_{+}^{2}(\mathrm{~K}) \oplus \Lambda_{-}^{2}(\mathrm{~K}) \oplus(\mathrm{K} \otimes \mathrm{L}) \oplus \Lambda_{+}^{2}(\mathrm{~L}) \oplus \Lambda_{-}^{2}(\mathrm{~L})
$$

We will refine both decompositions (3.3) and (3.4) into irreducible $\operatorname{Spin}^{h}(4)$-modules.

To begin, note first that as $\operatorname{Spin}^{h}(4)$-modules, we have that $\Lambda_{-}^{2}(\mathrm{~K}) \cong \mathrm{V}_{2,0,0}, \Lambda_{-}^{2}(\mathrm{~L}) \cong \mathrm{V}_{0,0,2}$, and $\Lambda_{+}^{2}(\mathrm{~K}) \cong \Lambda_{+}^{2}(\mathrm{~L}) \cong \mathrm{V}_{0,2,0}$ are irreducible. Thus, it remains only to decompose $\Lambda_{7}^{2}, \Lambda_{21}^{2}$, and $\mathrm{K} \otimes \mathrm{L}$. 
Definition 3.4. We define

$$
\begin{aligned}
& \left(\Lambda_{7}^{2}\right)_{0,2,0}:=\Lambda_{7}^{2} \cap\left(\Lambda_{+}^{2}(\mathrm{~K}) \oplus \Lambda_{+}^{2}(\mathrm{~L})\right), \\
& \left(\Lambda_{7}^{2}\right)_{1,0,1}:=\Lambda_{7}^{2} \cap(\mathrm{K} \otimes \mathrm{L}), \\
& \left(\Lambda_{21}^{2}\right)_{2,0,0}:=\Lambda_{21}^{2} \cap \Lambda_{-}^{2}(\mathrm{~K}), \\
& \left(\Lambda_{21}^{2}\right)_{0,0,2}:=\Lambda_{21}^{2} \cap \Lambda_{-}^{2}(\mathrm{~L}), \\
& \left(\Lambda_{21}^{2}\right)_{0,2,0}:=\Lambda_{21}^{2} \cap\left(\Lambda_{+}^{2}(\mathrm{~K}) \oplus \Lambda_{+}^{2}(\mathrm{~L})\right), \\
& \left(\Lambda_{21}^{2}\right)_{1,2,1}:=\Lambda_{21}^{2} \cap(\mathrm{K} \otimes \mathrm{L}) .
\end{aligned}
$$

The reader can check that, in fact, $\Lambda_{-}^{2}(\mathrm{~K}) \subset \Lambda_{21}^{2}$ and $\Lambda_{-}^{2}(\mathrm{~L}) \subset \Lambda_{21}^{2}$, so that $\left(\Lambda_{21}^{2}\right)_{2,0,0}=\Lambda_{-}^{2}(\mathrm{~K})$ and $\left(\Lambda_{21}^{2}\right)_{0,0,2}=\Lambda_{-}^{2}(\mathrm{~L})$.

Lemma 3.5. The decompositions

$$
\begin{aligned}
\Lambda_{7}^{2} & =\left(\Lambda_{7}^{2}\right)_{0,2,0} \oplus\left(\Lambda_{7}^{2}\right)_{1,0,1} \\
\Lambda_{21}^{2} & =\Lambda_{-}^{2}(\mathrm{~K}) \oplus\left(\Lambda_{21}^{2}\right)_{0,2,0} \oplus \Lambda_{-}^{2}(\mathrm{~L}) \oplus\left(\Lambda_{21}^{2}\right)_{1,2,1}
\end{aligned}
$$

consist of $\operatorname{Spin}^{h}(4)$-irreducible submodules.

Thus, the decomposition

$$
\Lambda^{2}(V)=\left[\left(\Lambda_{7}^{2}\right)_{0,2,0} \oplus\left(\Lambda_{7}^{2}\right)_{1,0,1}\right] \oplus\left[\Lambda_{-}^{2}(\mathrm{~K}) \oplus\left(\Lambda_{21}^{2}\right)_{0,2,0} \oplus \Lambda_{-}^{2}(\mathrm{~L}) \oplus\left(\Lambda_{21}^{2}\right)_{1,2,1}\right]
$$

refines (3.3), while the decomposition

$$
\Lambda^{2}(V)=\Lambda_{+}^{2}(\mathrm{~K}) \oplus \Lambda_{-}^{2}(\mathrm{~K}) \oplus\left[\left(\Lambda_{21}^{2}\right)_{1,2,1} \oplus\left(\Lambda_{7}^{2}\right)_{1,0,1}\right] \oplus \Lambda_{+}^{2}(\mathrm{~L}) \oplus \Lambda_{-}^{2}(\mathrm{~L})
$$

refines (3.4).

\subsubsection{Decomposition of 3-forms on $V$}

We now decompose $\Lambda^{3}\left(V^{*}\right)$ into $\operatorname{Spin}^{h}(4)$-irreducible submodules. As noted in $\S 3.1 .1$, the $\operatorname{Spin}(7)$-irreducible decomposition of $\Lambda^{3}\left(V^{*}\right)$ is

$$
\Lambda^{3}\left(V^{*}\right) \cong \Lambda_{8}^{3} \oplus \Lambda_{48}^{3}
$$

On the other hand, using $V^{*} \cong \mathrm{K} \oplus \mathrm{L}$, we also have the decomposition

$$
\Lambda^{3}\left(V^{*}\right) \cong \Lambda^{3}(\mathrm{~K}) \oplus\left(\left(\Lambda_{+}^{2}(\mathrm{~K}) \oplus \Lambda_{-}^{2}(\mathrm{~K})\right) \otimes \mathrm{L}\right) \oplus\left(\mathrm{K} \otimes\left(\Lambda_{+}^{2}(\mathrm{~L}) \oplus \Lambda_{-}^{2}(\mathrm{~L})\right)\right) \oplus \Lambda^{3}(\mathrm{~L}) .
$$

The summands $\Lambda^{3}(\mathrm{~K}) \cong \mathrm{K} \cong \mathrm{V}_{1,1,0}$ and $\Lambda^{3}(\mathrm{~L}) \cong \mathrm{L} \cong \mathrm{V}_{0,1,1}$ are irreducible, while the others are not. We have the following decompositions as $\operatorname{Spin}^{h}(4)$-modules:

$$
\begin{array}{ll}
\Lambda_{+}^{2}(\mathrm{~K}) \otimes \mathrm{L} \cong \mathrm{V}_{0,3,1} \oplus \mathrm{V}_{0,1,1}, & \mathrm{~K} \otimes \Lambda_{+}^{2}(\mathrm{~L}) \cong \mathrm{V}_{1,3,0} \oplus \mathrm{V}_{1,1,0}, \\
\Lambda_{-}^{2}(\mathrm{~K}) \otimes \mathrm{L} \cong \mathrm{V}_{2,1,1}, & \mathrm{~K} \otimes \Lambda_{-}^{2}(\mathrm{~L}) \cong \mathrm{V}_{1,1,2}
\end{array}
$$

We may use the $\operatorname{Spin}^{h}(4)$-invariant decomposition $V \cong \mathrm{K} \oplus \mathrm{L}$ to decompose the space $\Lambda_{8}^{3}$ into $\operatorname{Spin}^{h}(4)$-modules as $\Lambda_{8}^{3}=\left(\Lambda_{8}^{3}\right)_{\mathrm{K}} \oplus\left(\Lambda_{8}^{3}\right)_{\mathrm{L}}$, where

$$
\left(\Lambda_{8}^{3}\right)_{\mathrm{K}}:=\left\{*\left(\alpha \wedge \Phi_{0}\right): \alpha \in \mathrm{K}\right\} \cong \mathrm{K}, \quad\left(\Lambda_{8}^{3}\right)_{\mathrm{L}}:=\left\{*\left(\alpha \wedge \Phi_{0}\right): \alpha \in \mathrm{L}\right\} \cong \mathrm{L} .
$$

Comparing the decompositions (3.5) and (3.6) with the decompositions of their summands, it follows that the space $\Lambda_{48}^{3}$ decomposes into $\operatorname{Spin}^{h}(4)$-modules as

$$
\Lambda_{48}^{3} \cong \mathrm{V}_{0,3,1} \oplus \mathrm{V}_{2,1,1} \oplus \mathrm{V}_{1,3,0} \oplus \mathrm{V}_{1,1,2} \oplus \mathrm{V}_{0,1,1} \oplus \mathrm{V}_{1,1,0}
$$


Definition 3.6. Let $\left(\Lambda_{48}^{3}\right)_{i, j, k}$ denote the $V_{i, j, k}$ summand in the decomposition (3.7). We will also denote $\left(\Lambda_{48}^{3}\right)_{1,1,0}$ and $\left(\Lambda_{48}^{3}\right)_{0,1,1}$ by $\left(\Lambda_{48}^{3}\right)_{\mathrm{K}}$ and $\left(\Lambda_{48}^{3}\right)_{\mathrm{L}}$ respectively.

\subsection{The Refined Torsion Forms}

Let $\left(M^{8}, \Phi\right)$ be an 8-manifold with a $\operatorname{Spin}(7)$-structure. Fix a point $x \in M$, choose an arbitrary Cayley 4-plane $\mathrm{K}^{\sharp} \subset T_{x} M$, and let $\mathrm{L}^{\sharp} \subset T_{x} M$ denote its orthogonal 4-plane. Our purpose in this section is to understand how the torsion of the $\operatorname{Spin}(7)$-structure decomposes with respect to the splitting

$$
T_{x} M=\mathrm{K}^{\sharp} \oplus \mathrm{L}^{\sharp} \text {. }
$$

\subsubsection{The Refined Torsion Forms in a Local Spin ${ }^{h}(4)$ Frame}

Fix $x \in M^{8}$ and split $T_{x}^{*} M=\mathrm{K} \oplus \mathrm{L}$ as above. All calculations in this subsection a performed pointwise, and we suppress reference to $x \in M$. By the decompositions in $\S 3.2$, the torsion forms $\tau_{1}$ and $\tau_{3}$ decompose into $\operatorname{Spin}^{h}(4)$-irreducible pieces as follows:

$$
\begin{aligned}
& \tau_{1}=\left(\tau_{1}\right)_{\mathbf{K}}+\left(\tau_{1}\right)_{\mathbf{L}}, \\
& \tau_{3}=\left(\tau_{3}\right)_{\mathbf{K}}+\left(\tau_{3}\right)_{\mathbf{L}}+\left(\tau_{3}\right)_{0,3,1}+\left(\tau_{3}\right)_{2,1,1}+\left(\tau_{3}\right)_{1,3,0}+\left(\tau_{3}\right)_{1,1,2},
\end{aligned}
$$

where $\left(\tau_{1}\right)_{*} \in\left(\Lambda^{1}\right)_{*}$, and $\left(\tau_{3}\right)_{*} \in\left(\Lambda_{48}^{3}\right)_{*}$.

We refer to $\left(\tau_{1}\right)_{\mathrm{K}},\left(\tau_{1}\right)_{\mathrm{L}}, \ldots,\left(\tau_{3}\right)_{1,1,2}$ as the refined torsion forms of the Spin(7)-structure at $x$ relative to the splitting $T_{x}^{*} M=\mathrm{K} \oplus \mathrm{L}$.

We next express the refined torsion forms in terms of a local $\operatorname{Spin}^{h}(4)$-frame. Let $\left\{e_{1}, \ldots, e_{8}\right\}$ be an orthonormal basis for $T_{x} M$ for which $\mathrm{K}^{\sharp}=\operatorname{span}\left(e_{1}, e_{2}, e_{3}, e_{4}\right)$ and $\mathbf{L}^{\sharp}=\operatorname{span}\left(e_{5}, e_{6}, e_{7}, e_{8}\right)$. Let $\left\{e^{1}, \ldots, e^{8}\right\}$ denote the dual basis for $T_{x}^{*} M$.

Index Ranges: We will employ the following index ranges: $1 \leq p, q \leq 4$ and $4 \leq r, s \leq 8$ and $1 \leq a, b \leq 7$ and $1 \leq i, j, k, \ell, m \leq 8$ and $1 \leq \alpha, \beta \leq 12$.

Definition 3.7. Define the 2-forms

$$
\begin{array}{llll}
\Theta_{1}=e^{12}-e^{34}, & \Gamma_{1}=e^{12}+e^{34}, & \Omega_{1}=e^{56}-e^{78}, & \Upsilon_{1}=e^{56}+e^{78}, \\
\Theta_{2}=e^{13}+e^{24}, & \Gamma_{2}=e^{13}-e^{24}, & \Omega_{2}=e^{57}+e^{68}, & \Upsilon_{2}=e^{57}-e^{68}, \\
\Theta_{3}=e^{14}-e^{23}, & \Gamma_{3}=-e^{14}-e^{23}, & \Omega_{3}=-e^{58}+e^{67}, & \Upsilon_{3}=e^{58}+e^{67} .
\end{array}
$$

Lemma 3.8. We have that:

(a) $\left\{\Theta_{1}, \Theta_{2}, \Theta_{3}\right\}$ is a basis of $\left(\Lambda_{21}^{2}\right)_{2,0,0}$.

(b) $\left\{\Omega_{1}, \Omega_{2}, \Omega_{3}\right\}$ is a basis of $\left(\Lambda_{21}^{2}\right)_{0,0,2}$.

(c) $\left\{\Gamma_{1}-\Upsilon_{1}, \Gamma_{2}-\Upsilon_{2}, \Gamma_{3}-\Upsilon_{3}\right\}$ is a basis of $\left(\Lambda_{21}^{2}\right)_{0,2,0}$.

(d) $\left\{\Gamma_{1}-\Upsilon_{1}, \Gamma_{2}-\Upsilon_{2}, \Gamma_{3}-\Upsilon_{3}\right\}$ is a basis of $\left(\Lambda_{21}^{2}\right)_{0,2,0}$.

(e) $\left\{\Gamma_{1}+\Upsilon_{1}, \Gamma_{2}+\Upsilon_{2}, \Gamma_{3}+\Upsilon_{3}\right\}$ is a basis of $\left(\Lambda_{7}^{2}\right)_{0,2,0}$.

Definition 3.9. Define the 3 -forms

$$
\begin{gathered}
v_{i}=\iota_{e_{i}} \Phi_{0}, \\
\rho_{p}=v_{p}-7 *_{\mathrm{K}} e_{p}, \quad \rho_{r}=v_{r}-7 *_{\mathrm{L}} e_{r},
\end{gathered}
$$


and

$$
\begin{aligned}
& \mu_{1}=2\left(e^{5} \wedge \Theta_{3}+e^{6} \wedge \Theta_{2}\right), \\
& \nu_{1}=2\left(-e^{1} \wedge \Omega_{3}+e^{2} \wedge \Omega_{2}\right), \\
& \mu_{2}=2\left(-e^{5} \wedge \Theta_{2}+e^{6} \wedge \Theta_{3}\right), \\
& \nu_{2}=2\left(e^{1} \wedge \Omega_{2}+e^{2} \wedge \Omega_{3}\right) \text {, } \\
& \mu_{3}=2 e^{5} \wedge \Theta_{1}, \\
& \mu_{4}=2 e^{6} \wedge \Theta_{1}, \\
& \mu_{5}=2\left(-e^{5} \wedge \Theta_{3}+e^{6} \wedge \Theta_{2}\right), \\
& \nu_{3}=2\left(e^{3} \wedge \Omega_{3}-e^{4} \wedge \Omega_{2}\right) \text {, } \\
& \nu_{4}=2\left(-e^{3} \wedge \Omega_{2}-e^{4} \wedge \Omega_{3}\right) \text {, } \\
& \mu_{6}=2\left(-e^{5} \wedge \Theta_{2}-e^{6} \wedge \Theta_{3}\right), \\
& \nu_{5}=2\left(e^{3} \wedge \Omega_{3}+e^{4} \wedge \Omega_{2}\right) \text {, } \\
& \mu_{7}=2\left(e^{7} \wedge \Theta_{3}-e^{8} \wedge \Theta_{2}\right), \\
& \nu_{6}=2\left(-e^{3} \wedge \Omega_{2}+e^{4} \wedge \Omega_{3}\right) \text {, } \\
& \nu_{7}=2\left(e^{1} \wedge \Omega_{3}+e^{2} \wedge \Omega_{2}\right) \text {, } \\
& \mu_{8}=2\left(-e^{7} \wedge \Theta_{2}-e^{8} \wedge \Theta_{3}\right), \\
& \mu_{9}=2 e^{7} \wedge \Theta_{1}, \\
& \mu_{10}=-2 e^{8} \wedge \Theta_{1}, \\
& \mu_{11}=2\left(-e^{7} \wedge \Theta_{3}-e^{8} \wedge \Theta_{2}\right), \\
& \mu_{12}=2\left(-e^{7} \wedge \Theta_{2}+e^{8} \wedge \Theta_{3}\right) \text {, } \\
& \nu_{8}=2\left(-e^{1} \wedge \Omega_{2}+e^{2} \wedge \Omega_{3}\right) \text {, } \\
& \nu_{9}=-2 e^{1} \wedge \Omega_{1} \text {, } \\
& \nu_{10}=2 e^{2} \wedge \Omega_{1}, \\
& \nu_{11}=2 e^{3} \wedge \Omega_{1}, \\
& \nu_{12}=-2 e^{4} \wedge \Omega_{1}, \\
& \lambda_{1}=3\left(e^{5} \wedge \Gamma_{3}+e^{6} \wedge \Gamma_{2}\right), \\
& \lambda_{2}=3\left(-e^{5} \wedge \Gamma_{2}+e^{6} \wedge \Gamma_{3}\right) \text {, } \\
& \lambda_{3}=2 e^{5} \wedge \Gamma_{1}+e^{7} \wedge \Gamma_{3}-e^{8} \wedge \Gamma_{2}, \\
& \lambda_{4}=2 e^{6} \wedge \Gamma_{1}-e^{7} \wedge \Gamma_{2}-e^{8} \wedge \Gamma_{3}, \\
& \lambda_{5}=-e^{5} \wedge \Gamma_{3}+e^{6} \wedge \Gamma_{2}+2 e^{7} \wedge \Gamma_{1}, \\
& \lambda_{6}=-e^{5} \wedge \Gamma_{2}-e^{6} \wedge \Gamma_{3}-2 e^{8} \wedge \Gamma_{1}, \\
& \kappa_{1}=3\left(-e^{1} \wedge \Upsilon_{3}+e^{2} \wedge \Upsilon_{2}\right), \\
& \kappa_{2}=3\left(e^{1} \wedge \Upsilon_{2}+e^{2} \wedge \Upsilon_{3}\right) \text {, } \\
& \kappa_{3}=3\left(e^{3} \wedge \Upsilon_{3}-e^{4} \wedge \Upsilon_{2}\right), \\
& \kappa_{4}=3\left(-e^{3} \wedge \Upsilon_{2}-e^{4} \wedge \Upsilon_{3}\right) \text {, } \\
& \kappa_{5}=-2 e^{1} \wedge \Upsilon_{1}+e^{3} \wedge \Upsilon_{3}+e^{4} \wedge \Upsilon_{2} \text {, } \\
& \kappa_{6}=2 e^{1} \wedge \Upsilon_{1}-e^{3} \wedge \Upsilon_{2}+e^{4} \wedge \Upsilon_{3}, \\
& \lambda_{7}=3\left(-e^{7} \wedge \Gamma_{3}-e^{8} \wedge \Gamma_{2}\right) \text {, } \\
& \lambda_{8}=3\left(-e^{7} \wedge \Gamma_{2}+e^{8} \wedge \Gamma_{3}\right), \\
& \kappa_{7}=e^{1} \wedge \Upsilon_{3}+e^{2} \wedge \Upsilon_{2}+2 e^{3} \wedge \Upsilon_{1} \text {, } \\
& \kappa_{8}=-e^{1} \wedge \Upsilon_{2}+e^{2} \wedge \Upsilon_{3}-2 e^{4} \wedge \Upsilon_{1} \text {. }
\end{aligned}
$$

Lemma 3.10. We have that:

(a) $\left\{v_{p}: 1 \leq p \leq 4\right\}$ is a basis of $\left(\Lambda_{8}^{3}\right)_{1,1,0}$ and $\left\{v_{p+4}: 1 \leq p \leq 4\right\}$ is a basis of $\left(\Lambda_{8}^{3}\right)_{0,1,1}$.

(b) $\left\{\rho_{p}: 1 \leq p \leq 4\right\}$ is a basis of $\left(\Lambda_{48}^{3}\right)_{1,1,0}$ and $\left\{\rho_{p+4}: 1 \leq p \leq 4\right\}$ is a basis of $\left(\Lambda_{48}^{3}\right)_{0,1,1}$.

(c) $\left\{\mu_{a}: 1 \leq a \leq 12\right\}$ is a basis of $\left(\Lambda_{48}^{3}\right)_{2,1,1}$ and $\left\{\nu_{a}: 1 \leq a \leq 12\right\}$ is a basis of $\left(\Lambda_{48}^{3}\right)_{1,1,2}$.

(d) $\left\{\lambda_{i}: 1 \leq i \leq 8\right\}$ is basis of $\left(\Lambda_{48}^{3}\right)_{0,3,1}$ and $\left\{\kappa_{i}: 1 \leq i \leq 8\right\}$ is basis of $\left(\Lambda_{48}^{3}\right)_{1,3,0}$.

Definition 3.11. Let $\dagger$ denote the inverse of the isometric isomorphism

$$
\mathrm{L}^{\sharp} \rightarrow\left(\Lambda_{48}^{3}\right)_{\mathrm{L}}, \quad e_{r} \mapsto \frac{1}{\sqrt{42}} \rho_{r} .
$$

We now express the refined torsion forms in terms of the above bases. That is, we define functions $A_{p}, B_{r}$ and $C_{q}, D_{s}, E_{\alpha}, F_{\beta}, X_{i}, Y_{j}$ by

$$
\begin{aligned}
\left(\tau_{1}\right)_{\mathrm{K}}=32 A_{p} e^{p}, & \left(\tau_{3}\right)_{\mathrm{K}}=16 C_{q} \rho_{q} \\
\left(\tau_{1}\right)_{\mathrm{L}}=32 B_{r} e^{r}, & \left(\tau_{3}\right)_{\mathrm{L}}=16 D_{s} \rho_{s} \\
\left(\tau_{3}\right)_{2,1,1} & =8 E_{\alpha} \mu_{\alpha}, \\
\left(\tau_{3}\right)_{1,1,2} & =8 F_{\beta} \nu_{\beta}, \\
\left(\tau_{3}\right)_{0,3,1} & =8 X_{i} \lambda_{i}, \\
\left(\tau_{3}\right)_{1,3,0} & =8 Y_{j} \kappa_{j} .
\end{aligned}
$$

For future use, we record the formulae

$$
\left[\left(\tau_{1}\right)_{\mathrm{L}}\right]^{\sharp}=32 B_{r} e_{r}, \quad\left[\left(\tau_{3}\right)_{\mathrm{L}}\right]^{\dagger}=16 \sqrt{42} D_{s} e_{s} .
$$




\subsubsection{The Torsion Functions $T_{a i}$}

Let $\left(M^{8}, \Phi\right)$ be an 8-manifold with a Spin(7)-structure, and let $g_{\Phi}$ denote the underlying Riemannian metric. Let $F_{\mathrm{SO}(8)} \rightarrow M$ denote the oriented orthonormal coframe bundle of $g_{\Phi}$, and let $\omega=\left(\omega_{1}, \ldots, \omega_{8}\right) \in \Omega^{1}\left(F_{S O(8)} ; V\right)$ denote the tautological 1-form. By the Fundamental Lemma of Riemannian Geometry, there exists a unique 1-form $\psi \in \Omega^{1}\left(F_{\mathrm{SO}(8)} ; \mathfrak{s o}(8)\right)$, the Levi-Civita connection form of $g_{\Phi}$, satisfying the First Structure Equation

$$
d \omega=-\psi \wedge \omega
$$

Let $\pi: F_{\mathrm{Spin}(7)} \rightarrow M$ denote the $\operatorname{Spin}(7)$-coframe bundle of $M$. Restricted to $F_{\mathrm{Spin}(7)} \subset F_{\mathrm{SO}(8)}$, the Levi-Civita 1-form $\psi$ is no longer a connection 1-form. Indeed, according to the Spin(7)invariant splitting $\mathfrak{s o}(8)=\mathfrak{s p i n}(7) \oplus \mathbb{R}^{7}$, we have the decomposition

$$
\psi=\theta+2 \gamma
$$

where $\theta=\left(\theta_{i j}\right) \in \Omega^{1}\left(F_{\operatorname{Spin}(7)} ; \mathfrak{s p i n}(7)\right)$ is a connection 1-form (the so-called natural connection of the $\operatorname{Spin}(7)$-structure $\Phi)$ and $\gamma \in \Omega\left(F_{\operatorname{Spin}(7)} ; \mathbb{R}^{7}\right)$ is a $\pi$-semibasic 1 -form. The inclusion $\mathbb{R}^{7} \hookrightarrow \mathfrak{s o}(8)$ is given by

$$
\left(\gamma_{1}, \ldots \gamma_{7}\right) \mapsto\left[\begin{array}{cccc|cccc}
0 & \gamma_{1} & \gamma_{2} & \gamma_{3} & \gamma_{4} & \gamma_{5} & \gamma_{6} & \gamma_{7} \\
-\gamma_{1} & 0 & \gamma_{3} & -\gamma_{2} & \gamma_{5} & -\gamma_{4} & \gamma_{7} & -\gamma_{6} \\
-\gamma_{2} & -\gamma_{3} & 0 & \gamma_{1} & \gamma_{6} & -\gamma_{7} & -\gamma_{4} & \gamma_{5} \\
-\gamma_{3} & \gamma_{2} & -\gamma_{1} & 0 & -\gamma_{7} & -\gamma_{6} & \gamma_{5} & \gamma_{4} \\
\hline-\gamma_{4} & -\gamma_{5} & -\gamma_{6} & \gamma_{7} & 0 & \gamma_{1} & \gamma_{2} & -\gamma_{3} \\
-\gamma_{5} & \gamma_{4} & \gamma_{7} & \gamma_{6} & -\gamma_{1} & 0 & -\gamma_{3} & -\gamma_{2} \\
-\gamma_{6} & -\gamma_{7} & \gamma_{4} & -\gamma_{5} & -\gamma_{2} & \gamma_{3} & 0 & \gamma_{1} \\
-\gamma_{7} & \gamma_{6} & -\gamma_{5} & -\gamma_{4} & \gamma_{3} & \gamma_{2} & -\gamma_{1} & 0
\end{array}\right]
$$

Since $\gamma$ is $\pi$-semibasic, we may write

$$
\gamma_{a}=T_{a i} \omega_{i}, \text { for } 1 \leq a \leq 7
$$

for some matrix-valued function $T=\left(T_{a i}\right): F_{\operatorname{Spin}(7)} \rightarrow \operatorname{Hom}\left(V, \mathbb{R}^{7}\right)$. The functions $T_{a i}$ encode the torsion of the Spin(7)-structure: they are equivalent to the torsion forms $\tau_{1}$ and $\tau_{3}$ via the isomorphism

$$
\operatorname{Hom}\left(V, \mathbb{R}^{7}\right) \cong \Lambda_{8}^{1} \oplus \Lambda_{48}^{3}
$$

\subsubsection{Decomposition of the Torsion Functions}

For our computations in $\S 3.4$, we will need to express the torsion function $T_{a i}$ in terms of the functions $A_{p}, B_{r}, \ldots, Y_{j}$. To this end, we continue to work on the total space of the Spin(7)-coframe

bundle $\pi: F_{\operatorname{Spin}(7)} \rightarrow M$, pulling back all quantities defined on $M$ to $F_{\operatorname{Spin}(7)}$. Following common convention, and similarly to $\S 2.3 .3$, we omit $\pi^{*}$ from the notation.

The torsion forms $\tau_{1}$ and $\tau_{3}$ satisfy

$$
d \Phi=\tau_{1} \wedge \Phi+* \tau_{3} .
$$

Into the left-hand side we substitute $\Phi=\frac{1}{24} \Phi_{i j k l} \omega_{i j k l}$ and use the first structure equation (3.11), together with the decomposition (3.12), to express the $d \omega$ terms in terms of the torsion functions 
$T_{a i}$. Into the right-hand side we again substitute $\Phi=\frac{1}{24} \Phi_{i j k l} \omega_{i j k l}$, as well as the expansions (3.8) and (3.9).

Upon equating coefficients, we obtain a system of 56 linear equations relating the 56 functions $T_{a i}$ on the left-hand side to the 56 functions $A_{p}, B_{r}, \ldots, Y_{j}$ on the right-hand side. One can then use a computer algebra system (we have used MAPLE) to solve this linear system for the $T_{a i}$. We now exhibit the result, taking advantage of the $\operatorname{Spin}^{h}(4)$-invariant isomorphism

$$
\operatorname{Hom}\left(V, \mathbb{R}^{7}\right) \cong\left(\mathrm{V}_{0,2,0} \oplus \mathrm{V}_{1,0,1}\right) \otimes(\mathrm{K} \oplus \mathrm{L})
$$

to highlight the structure of the solution.

We find

$$
\begin{aligned}
{\left[\begin{array}{llll}
T_{11} & T_{12} & T_{13} & T_{14} \\
T_{21} & T_{22} & T_{23} & T_{24} \\
T_{31} & T_{32} & T_{33} & T_{34}
\end{array}\right]=} & {\left[\begin{array}{cccc}
2 Y_{5} & -2 Y_{6} & -2 Y_{7} & 2 Y_{8} \\
Y_{8}-3 Y_{2} & -3 Y_{1}-Y_{7} & 3 Y_{4}+Y_{6} & 3 Y_{3}-Y_{5} \\
-3 Y_{1}+Y_{7} & Y_{8}+3 Y_{2} & 3 Y_{3}+Y_{5} & -3 Y_{4}+Y_{6}
\end{array}\right] } \\
& +\left[\begin{array}{cccc}
-4 C_{2}+A_{2} & 4 C_{1}-A_{1} & -4 C_{4}+A_{4} & 4 C_{3}-A_{3} \\
-4 C_{3}+A_{3} & 4 C_{4}-A_{4} & 4 C_{1}-A_{1} & -4 C_{2}+A_{2} \\
-4 C_{4}+A_{4} & -4 C_{3}+A_{3} & 4 C_{2}-A_{2} & 4 C_{1}-A_{1}
\end{array}\right],
\end{aligned}
$$

corresponding to $\mathrm{V}_{0,2,0} \otimes \mathrm{K} \cong \mathrm{V}_{1,3,0} \oplus \mathrm{V}_{1,1,0}$, and

$$
\begin{aligned}
{\left[\begin{array}{llll}
T_{15} & T_{16} & T_{17} & T_{18} \\
T_{25} & T_{26} & T_{27} & T_{28} \\
T_{35} & T_{36} & T_{37} & T_{38}
\end{array}\right]=} & {\left[\begin{array}{cccc}
-2 X_{3} & -2 X_{4} & -2 X_{5} & 2 X_{6} \\
X_{6}+3 X_{2} & -X_{5}-3 X_{1} & X_{4}+3 X_{8} & X_{3}+3 X_{7} \\
-X_{5}+3 X_{1} & -X_{6}+3 X_{2} & X_{3}-3 X_{7} & -X_{4}+3 X_{8}
\end{array}\right] } \\
& +\left[\begin{array}{cccc}
-4 D_{2}+B_{2} & 4 D_{1}-B_{1} & -4 D_{4}+B_{4} & 4 D_{3}-B_{3} \\
-4 D_{3}+B_{3} & 4 D_{4}-B_{4} & 4 D_{1}-B_{1} & -4 D_{2}+B_{2} \\
4 D_{4}-B_{4} & 4 D_{3}-B_{3} & -4 D_{2}+B_{2} & -4 D_{1}+B_{1}
\end{array}\right],
\end{aligned}
$$

corresponding to $\mathrm{V}_{0,2,0} \otimes \mathrm{L} \cong \mathrm{V}_{0,3,1} \oplus \mathrm{V}_{0,1,1}$, and

$$
\begin{aligned}
{\left[\begin{array}{llll}
T_{41} & T_{42} & T_{43} & T_{44} \\
T_{51} & T_{52} & T_{53} & T_{54} \\
T_{61} & T_{62} & T_{63} & T_{64} \\
T_{71} & T_{72} & T_{73} & T_{74}
\end{array}\right]=} & {\left[\begin{array}{cccc}
-2 E_{12}+E_{4} & 2 E_{11}+E_{3} & -2 E_{6}-E_{10} & -2 E_{5}+E_{9} \\
2 E_{11}-E_{3} & 2 E_{12}+E_{4} & 2 E_{5}+E_{9} & -2 E_{6}+E_{10} \\
2 E_{2}-E_{10} & 2 E_{1}+E_{9} & -2 E_{8}-E_{4} & 2 E_{7}-E_{3} \\
-E_{9}+2 E_{1} & -2 E_{2}-E_{10} & -2 E_{7}-E_{3} & -2 E_{8}+E_{4}
\end{array}\right] } \\
+ & {\left[\begin{array}{cccc}
3 D_{1}+B_{1} & -3 D_{2}-B_{2} & -3 D_{3}-B_{3} & 3 D_{4}+B_{4} \\
3 D_{2}+B_{2} & 3 D_{1}+B_{1} & 3 D_{4}+B_{4} & 3 D_{3}+B_{3} \\
3 D_{3}+B_{3} & -3 D_{4}-B_{4} & 3 D_{1}+B_{1} & -3 D_{2}-B_{2} \\
3 D_{4}+B_{4} & 3 D_{3}+B_{3} & -3 D_{2}-B_{2} & -3 D_{1}-B_{1}
\end{array}\right] }
\end{aligned}
$$

corresponding to $\mathrm{V}_{1,0,1} \otimes \mathrm{K} \cong \mathrm{V}_{2,1,1} \oplus \mathrm{V}_{0,1,1}$, and

$$
\begin{aligned}
{\left[\begin{array}{llll}
T_{45} & T_{46} & T_{47} & T_{48} \\
T_{55} & T_{56} & T_{57} & T_{58} \\
T_{65} & T_{66} & T_{67} & T_{68} \\
T_{75} & T_{76} & T_{77} & T_{78}
\end{array}\right]=} & {\left[\begin{array}{cccc}
2 F_{4}-F_{10} & -2 F_{3}+F_{9} & 2 F_{8}+F_{12} & 2 F_{7}-F_{11} \\
-2 F_{3}-F_{9} & -2 F_{4}-F_{10} & -2 F_{7}-F_{11} & 2 F_{8}-F_{12} \\
2 F_{2}+F_{12} & -2 F_{1}-F_{11} & 2 F_{6}+F_{10} & 2 F_{5}-F_{9} \\
2 F_{1}-F_{11} & 2 F_{2}-F_{12} & 2 F_{5}+F_{9} & F_{10}-2 F_{6}
\end{array}\right] } \\
+ & {\left[\begin{array}{ccccc}
-3 C_{1}-A_{1} & 3 C_{2}+A_{2} & 3 C_{3}+A_{3} & -3 C_{4}-A_{4} \\
-3 C_{2}-A_{2} & -3 C_{1}-A_{1} & -3 C_{4}-A_{4} & -3 C_{3}-A_{3} \\
-3 C_{3}-A_{3} & 3 C_{4}+A_{4} & -3 C_{1}-A_{1} & 3 C_{2}+A_{2} \\
3 C_{4}+A_{4} & 3 C_{3}+A_{3} & -3 C_{2}-A_{2} & -3 C_{1}-A_{1}
\end{array}\right], }
\end{aligned}
$$

corresponding to $\mathrm{V}_{1,0,1} \otimes \mathrm{L} \cong \mathrm{V}_{1,1,2} \oplus \mathrm{V}_{1,1,0}$. 


\subsection{Mean Curvature of Cayley 4-Folds}

In this subsection we derive a formula (Theorem 3.12) for the mean curvature of a Cayley 4 -fold in an arbitrary 8-manifold $(M, \Phi)$ with $\operatorname{Spin}(7)$-structure.

We continue with the notation of $\S 3.3$, letting $\pi: F_{\operatorname{Spin}(7)} \rightarrow M$ denote the $\operatorname{Spin}(7)$-coframe bundle of $M$ and $\omega=\left(\omega_{\mathrm{K}}, \omega_{\mathrm{L}}\right) \in \Omega^{1}\left(F_{\mathrm{Spin}(7)} ; \mathrm{K}^{\sharp} \oplus \mathrm{L}^{\sharp}\right)$ denote the tautological 1-form. We remind the reader that $\theta=\left(\theta_{i j}\right) \in \Omega^{1}\left(F_{\operatorname{Spin}(7)} ; \mathfrak{s p i n}(7)\right)$ is the natural connection 1-form, and that $\gamma=$ $\left(\gamma_{i j}\right) \in \Omega^{1}\left(F_{\operatorname{Spin}(7)} ; \mathbb{R}^{7}\right)$ is a $\pi$-semibasic 1 -form encoding the torsion of $\Phi$. Here $\gamma_{i j}$ refers to the image of $\left(\gamma_{1}, \ldots, \gamma_{7}\right)$ under the map $\mathbb{R}^{7} \rightarrow \mathfrak{s o}(8)$ defined by $(3.13)$. We have $\gamma_{a}=T_{a i} \omega_{i}$ for $T=\left(T_{a i}\right): F_{\operatorname{Spin}(7)} \rightarrow \operatorname{Hom}\left(V, \mathbb{R}^{7}\right)$.

Let $f: \Sigma^{4} \rightarrow M$ be an immersion of a Cayley 4 -form into $M$, and let $f^{*}\left(F_{\operatorname{Spin}(7)}\right) \rightarrow \Sigma$ denote the pullback bundle. Let $B \subset f^{*}\left(F_{\operatorname{Spin}(7)}\right)$ denote the subbundle of coframes adapted to $\Sigma$, i.e., the subbundle whose fibre over $x \in \Sigma$ is

$$
\left.B\right|_{x}=\left\{\left.u \in f^{*}\left(F_{\operatorname{Spin}(7)}\right)\right|_{x}: u\left(T_{x} \Sigma\right)=\mathrm{K}^{\sharp} \oplus 0\right\} .
$$

We recall (Proposition 3.3) that Spin(7) acts transitively on the set of Cayley 4-planes, with stabilizer isomorphic to $\operatorname{Spin}^{h}(4)$. It follows that $B$ is a well-defined $\operatorname{Spin}^{h}(4)$-bundle over $\Sigma$. Note that on $B$ we have

$$
\omega_{\mathrm{L}}=0 \text {. }
$$

We may exploit the splitting $T_{x} M=T_{x} \Sigma \oplus\left(T_{x} \Sigma\right)^{\perp} \cong \mathrm{K}^{\sharp} \oplus \mathrm{L}^{\sharp}$ to decompose $\theta$ and $\gamma$ into $\operatorname{Spin}^{h}(4)$-irreducible pieces. To decompose the connection 1-form $\theta \in \Omega^{1}(B ; \mathfrak{s p i n}(7))$, we split

$$
\mathfrak{s p i n}(7) \cong \Lambda_{21}^{2} \cong \Lambda_{-}^{2}(\mathrm{~K}) \oplus\left(\Lambda_{21}^{2}\right)_{0,2,0} \oplus \Lambda_{-}^{2}(\mathrm{~L}) \oplus\left(\Lambda_{21}^{2}\right)_{1,2,1}
$$

so that $\theta$ takes the block form

$$
\begin{aligned}
\theta & =\left[\begin{array}{ccc|cccc}
\chi+\rho_{\mathrm{K}}(\zeta) & -\sigma^{T} \\
\sigma & \xi+\rho_{\mathrm{L}}(\zeta)
\end{array}\right] \\
& =\left[\begin{array}{cccc|cccc}
0 & \chi_{1}+\zeta_{1} & \chi_{2}+\zeta_{2} & \chi_{3}-\zeta_{3} & 2 \sigma_{1}-\sigma_{7} & 2 \sigma_{2}-\sigma_{8} & \sigma_{5}-2 \sigma_{11} & -\sigma_{6}+2 \sigma_{12} \\
-\chi_{1}-\zeta_{1} & 0 & -\chi_{3}-\zeta_{3} & \chi_{2}-\zeta_{2} & -2 \sigma_{2}-\sigma_{8} & 2 \sigma_{1}+\sigma_{7} & -\sigma_{6}-2 \sigma_{12} & -\sigma_{5}-2 \sigma_{11} \\
-\chi_{2}-\zeta_{2} & \chi_{3}+\zeta_{3} & 0 & -\chi_{1}+\zeta_{1} & -2 \sigma_{3}-\sigma_{5} & -2 \sigma_{4}-\sigma_{6} & -\sigma_{7}-2 \sigma_{9} & \sigma_{8}+2 \sigma_{10} \\
-\chi_{3}+\zeta_{3} & -\chi_{2}+\zeta_{2} & \chi_{1}-\zeta_{1} & 0 & 2 \sigma_{4}-\sigma_{6} & -2 \sigma_{3}+\sigma_{5} & \sigma_{8}-2 \sigma_{10} & \sigma_{7}-2 \sigma_{9} \\
\hline-2 \sigma_{1}+\sigma_{7} & 2 \sigma_{2}+\sigma_{8} & 2 \sigma_{3}+\sigma_{5} & -2 \sigma_{4}+\sigma_{6} & 0 & -\xi_{1}-\zeta_{1} & -\xi_{2}-\zeta_{2} & \xi_{3}-\zeta_{3} \\
-2 \sigma_{2}+\sigma_{8} & -2 \sigma_{1}-\sigma_{7} & 2 \sigma_{4}+\sigma_{6} & 2 \sigma_{3}-\sigma_{5} & \xi_{1}+\zeta_{1} & 0 & -\xi_{3}-\zeta_{3} & -\xi_{2}+\zeta_{2} \\
-\sigma_{5}+2 \sigma_{11} & \sigma_{6}+2 \sigma_{12} & \sigma_{7}+2 \sigma_{9} & -\sigma_{8}+2 \sigma_{10} & \xi_{2}+\zeta_{2} & \xi_{3}+\zeta_{3} & 0 & \xi_{1}-\zeta_{1} \\
\sigma_{6}-2 \sigma_{12} & \sigma_{5}+2 \sigma_{11} & -\sigma_{8}-2 \sigma_{10} & -\sigma_{7}+2 \sigma_{9} & -\xi_{3}+\zeta_{3} & \xi_{2}-\zeta_{2} & -\xi_{1}+\zeta_{1} & 0
\end{array}\right] .
\end{aligned}
$$

Similarly, the 1 -form $\gamma \in \Omega^{1}\left(B ; \mathbb{R}^{7}\right)$ breaks into block form as

$$
\gamma=\left[\begin{array}{cc}
\phi_{\mathrm{K}}\left(\gamma_{0,2,0}\right) & -\left(\gamma_{1,0,1}\right)^{T} \\
\gamma_{1,0,1} & \phi_{\mathrm{L}}\left(\gamma_{0,2,0}\right)
\end{array}\right]=\left[\begin{array}{cccc|cccc}
0 & \gamma_{1} & \gamma_{2} & \gamma_{3} & \gamma_{4} & \gamma_{5} & \gamma_{6} & \gamma_{7} \\
-\gamma_{1} & 0 & \gamma_{3} & -\gamma_{2} & \gamma_{5} & -\gamma_{4} & \gamma_{7} & -\gamma_{6} \\
-\gamma_{2} & -\gamma_{3} & 0 & \gamma_{1} & \gamma_{6} & -\gamma_{7} & -\gamma_{4} & \gamma_{5} \\
-\gamma_{3} & \gamma_{2} & -\gamma_{1} & 0 & -\gamma_{7} & -\gamma_{6} & \gamma_{5} & \gamma_{4} \\
\hline-\gamma_{4} & -\gamma_{5} & -\gamma_{6} & \gamma_{7} & 0 & \gamma_{1} & \gamma_{2} & -\gamma_{3} \\
-\gamma_{5} & \gamma_{4} & \gamma_{7} & \gamma_{6} & -\gamma_{1} & 0 & -\gamma_{3} & -\gamma_{2} \\
-\gamma_{6} & -\gamma_{7} & \gamma_{4} & -\gamma_{5} & -\gamma_{2} & \gamma_{3} & 0 & \gamma_{1} \\
-\gamma_{7} & \gamma_{6} & -\gamma_{5} & -\gamma_{4} & \gamma_{3} & \gamma_{2} & -\gamma_{1} & 0
\end{array}\right]
$$


In this notation, the first structure equation (3.11) on $B$ reads

$$
d\left(\begin{array}{c}
\omega_{\mathrm{K}} \\
0
\end{array}\right)=-\left(\left[\begin{array}{cc}
\chi+\rho_{\mathrm{K}}(\zeta) & -\sigma^{T} \\
\sigma & \xi+\rho_{\mathrm{L}}(\zeta)
\end{array}\right]+2\left[\begin{array}{cc}
\phi_{\mathrm{K}}\left(\gamma_{0,2,0}\right) & -\left(\gamma_{1,0,1}\right)^{T} \\
\gamma_{1,0,1} & \phi_{\mathrm{L}}\left(\gamma_{0,2,0}\right)
\end{array}\right]\right) \wedge\left(\begin{array}{c}
\omega_{\mathrm{K}} \\
0
\end{array}\right)
$$

In particular, the second line gives

$$
\left(\sigma+2 \gamma_{1,0,1}\right) \wedge \omega_{\mathrm{K}}=0
$$

or in detail

$$
\begin{aligned}
& {\left[\begin{array}{cccc}
-2 \sigma_{1}+\sigma_{7} & 2 \sigma_{2}+\sigma_{8} & 2 \sigma_{3}+\sigma_{5} & -2 \sigma_{4}+\sigma_{6} \\
-2 \sigma_{2}+\sigma_{8} & -2 \sigma_{1}-\sigma_{7} & 2 \sigma_{4}+\sigma_{6} & 2 \sigma_{3}-\sigma_{5} \\
-\sigma_{5}+2 \sigma_{11} & \sigma_{6}+2 \sigma_{12} & \sigma_{7}+2 \sigma_{9} & -\sigma_{8}+2 \sigma_{10} \\
\sigma_{6}-2 \sigma_{12} & \sigma_{5}+2 \sigma_{11} & -\sigma_{8}-2 \sigma_{10} & -\sigma_{7}+2 \sigma_{9}
\end{array}\right] \wedge\left[\begin{array}{l}
\omega_{1} \\
\omega_{2} \\
\omega_{3} \\
\omega_{4}
\end{array}\right]} \\
& =-2\left[\begin{array}{cccc}
-\gamma_{4} & -\gamma_{5} & -\gamma_{6} & \gamma_{7} \\
-\gamma_{5} & \gamma_{4} & \gamma_{7} & \gamma_{6} \\
-\gamma_{6} & -\gamma_{7} & \gamma_{4} & -\gamma_{5} \\
-\gamma_{7} & \gamma_{6} & -\gamma_{5} & -\gamma_{4}
\end{array}\right] \wedge\left[\begin{array}{l}
\omega_{1} \\
\omega_{2} \\
\omega_{3} \\
\omega_{4}
\end{array}\right] .
\end{aligned}
$$

Note that on $B$ the 1 -forms $\sigma$ and $\gamma$ are semibasic. We have

$$
\sigma_{\alpha}=S_{\alpha p} \omega_{p}, \quad \gamma_{r}=T_{r q} \omega_{q}
$$

for some function $S=\left(S_{\alpha p}\right): B \rightarrow \mathrm{V}_{1,2,1} \otimes \mathrm{K}$, recalling our index ranges $1 \leq \alpha \leq 12$ and $1 \leq p, q \leq 4$ and $4 \leq r \leq 8$.

Now, the 48 functions $S_{\alpha p}$ and the 16 functions $T_{r q}$ are not independent: equation (3.17) amounts to 24 linear relations among them, 8 of which involve only the functions $S_{\alpha p}$. In our calculation of the mean curvature vector of a Cayley 4 -fold we shall require only the following 4 of the relations:

$$
\left[\begin{array}{c}
S_{82}-2 S_{33}+2 S_{44}-S_{53}+2 S_{11}-2 S_{22}-S_{64}-S_{71} \\
-S_{81}-2 S_{34}-2 S_{43}+S_{54}+2 S_{12}+2 S_{21}-S_{63}+S_{72} \\
S_{84}-2 S_{111}-2 S_{122}+S_{51}-2 S_{93}-2 S_{104}-S_{62}-S_{73} \\
S_{83}-2 S_{112}+2 S_{121}-S_{52}-2 S_{94}+2 S_{103}-S_{61}+S_{74}
\end{array}\right]=6\left[\begin{array}{c}
T_{74}+T_{41}+T_{52}+T_{63} \\
-T_{73}-T_{42}+T_{51}-T_{64} \\
T_{72}-T_{43}+T_{54}+T_{61} \\
T_{71}+T_{44}+T_{53}-T_{62}
\end{array}\right]
$$

Theorem 3.12. Let $\Sigma \subset M$ be a Cayley 4-fold immersed in an 8-manifold $M$ with Spin(7)structure. Then the mean curvature vector $H$ of $\Sigma$ is given by

$$
H=-\left[\left(\tau_{1}\right)_{\mathrm{L}}\right]^{\sharp}-\frac{\sqrt{42}}{7}\left[\left(\tau_{3}\right)_{\mathrm{L}}\right]^{\dagger} .
$$

In particular, the largest torsion class of Spin(7)-structures for which every Cayley 4-fold is minimal is the class of torsion-free Spin(7)-structures.

Proof. Let $\beta_{p}=*_{\mathrm{K}} \omega_{p} \in \Omega^{3}(B)$, and let $\operatorname{vol}_{\mathrm{K}}=\omega_{1234}$. The components of the mean curvature 
vector $H$ of $\Sigma$ may be computed as follows:

$$
\begin{aligned}
& {\left[\begin{array}{l}
H_{5} \\
H_{6} \\
H_{7} \\
H_{8}
\end{array}\right] \text { vol }_{\mathrm{K}}=} {\left[\begin{array}{llll}
\psi_{51} & \psi_{52} & \psi_{53} & \psi_{54} \\
\psi_{61} & \psi_{62} & \psi_{63} & \psi_{64} \\
\psi_{71} & \psi_{72} & \psi_{73} & \psi_{74} \\
\psi_{81} & \psi_{82} & \psi_{83} & \psi_{84}
\end{array}\right] \wedge\left[\begin{array}{l}
\beta_{1} \\
\beta_{2} \\
\beta_{3} \\
\beta_{4}
\end{array}\right] } \\
&= {\left[\begin{array}{cccc}
-2 \sigma_{1}+\sigma_{7} & 2 \sigma_{2}+\sigma_{8} & 2 \sigma_{3}+\sigma_{5} & -2 \sigma_{4}+\sigma_{6} \\
-2 \sigma_{2}+\sigma_{8} & -2 \sigma_{1}-\sigma_{7} & 2 \sigma_{4}+\sigma_{6} & 2 \sigma_{3}-\sigma_{5} \\
-\sigma_{5}+2 \sigma_{11} & \sigma_{6}+2 \sigma_{12} & \sigma_{7}+2 \sigma_{9} & -\sigma_{8}+2 \sigma_{10} \\
\sigma_{6}-2 \sigma_{12} & \sigma_{5}+2 \sigma_{11} & -\sigma_{8}-2 \sigma_{10} & -\sigma_{7}+2 \sigma_{9}
\end{array}\right] \wedge\left[\begin{array}{l}
\beta_{1} \\
\beta_{2} \\
\beta_{3} \\
\beta_{4}
\end{array}\right] } \\
&+2\left[\begin{array}{cccc}
-\gamma_{4} & -\gamma_{5} & -\gamma_{6} & \gamma_{7} \\
-\gamma_{5} & \gamma_{4} & \gamma_{7} & \gamma_{6} \\
-\gamma_{6} & -\gamma_{7} & \gamma_{4} & -\gamma_{5} \\
-\gamma_{7} & \gamma_{6} & -\gamma_{5} & -\gamma_{4}
\end{array}\right] \wedge\left[\begin{array}{l}
\beta_{1} \\
\beta_{2} \\
\beta_{3} \\
\beta_{4}
\end{array}\right] .
\end{aligned}
$$

To evaluate the first term in (3.19), we substitute $\sigma_{\alpha}=S_{\alpha p} \omega_{p}$, followed by (3.18), and finally (3.14), to obtain

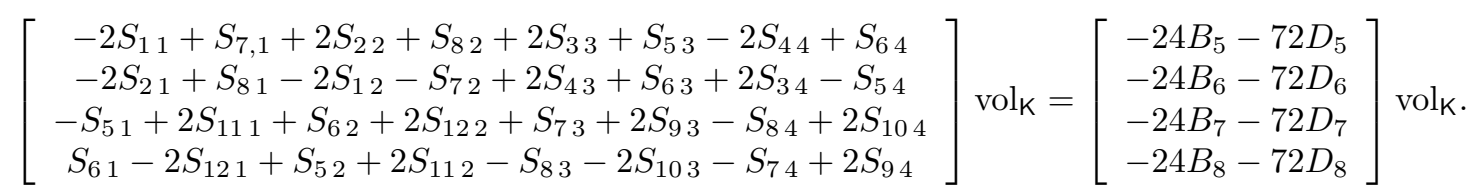

Similarly, to evaluate the second term in (3.19), we substitute $\gamma_{r}=T_{r q} \omega_{q}$, followed by (3.14), to obtain:

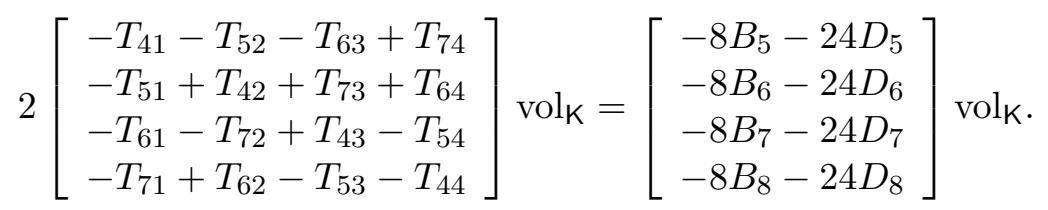

We conclude that $H_{r}=-32 B_{r}-96 D_{r}$, and so (3.10) yields that

$$
H=-\left[\left(\tau_{1}\right)_{\mathrm{L}}\right]^{\sharp}-\frac{\sqrt{42}}{7}\left[\left(\tau_{3}\right)_{\mathrm{L}}\right]^{\dagger}
$$

\subsubsection{The Second Fundamental Form of a Cayley 4-fold}

The mean curvature vector is not the only part of the second fundamental form of a Cayley 4 -fold that can be written in terms of the torsion forms of the ambient Spin(7)-structure. For a Cayley 4-fold, the second fundamental form is naturally a section of a vector bundle modeled on the $\operatorname{Spin}^{h}(4)$-representation

$$
\begin{aligned}
\operatorname{Sym}^{2}(\mathrm{~K}) \otimes \mathrm{L} & \cong\left(\mathrm{V}_{2,2,0} \oplus \mathbb{R}\right) \otimes \mathrm{L} \\
& \cong \mathrm{V}_{2,3,1} \oplus \mathrm{V}_{0,3,1} \oplus \mathrm{V}_{2,1,1} \oplus \mathrm{L},
\end{aligned}
$$

and so the second fundamental form naturally decomposes into four pieces, with the piece corresponding to the $\mathrm{L}$ summand equal to the mean curvature vector.

By calculations similar to those performed in the proof of Theorem 3.12, it is possible to show that the piece of the second fundamental form corresponding to the $\mathrm{V}_{0,3,1}$ summand in (3.20) is

identically zero, while the piece corresponding to the $\mathrm{V}_{2,1,1}$ summand in (3.20) is proportional to the refined torsion form $\left(\tau_{3}\right)_{2,1,1}$. 


\section{References}

[1] B.S. Acharya. On mirror symmetry for manifolds of exceptional holonomy. Nuclear Physics $B, 524(1): 269-282,1998$.

[2] Gavin Ball and Jesse Madnick. The Mean Curvature of Special Lagrangian 3-folds in SU(3)Structures with Torsion. arXiv e-prints, page arXiv:1905.03713, September 2019.

[3] Katrin Becker, Melanie Becker, David R. Morrison, Hirosi Ooguri, Yaron Oz, and Zheng Yin. Supersymmetric cycles in exceptional holonomy manifolds and Calabi-Yau four-folds. Nuclear Physics B, 480(1):225 - 238, 1996.

[4] Robert L. Bryant. Metrics with Exceptional Holonomy. Annals of Mathematics, 126(3):525$576,1987$.

[5] Robert L. Bryant. Some remarks on $G_{2}$-structures. Proceedings of Gökova Geometry-Topology Confernece 2005, 2006.

[6] Marisa Fernández. A classification of Riemannian manifolds with structure group Spin(7). Annali di Matematica Pura ed Applicata, 143(1):101-122, Dec 1986.

[7] Robert B. Gardner. The method of equivalence and its applications, volume 58 of CBMSNSF Regional Conference Series in Applied Mathematics. Society for Industrial and Applied Mathematics (SIAM), Philadelphia, PA, 1989.

[8] Sergei Gukov, Shing-Tung Yau, and Eric Zaslow. Duality and fibrations on $G_{2}$ manifolds. Turk. J. Math., 27(1):61-97, 2003.

[9] Reese Harvey and H Blaine Lawson. Calibrated geometries. Acta Mathematica, 148(1):47-157, 1982.

[10] Paul Reynolds. On Conformal Submersions and Manifolds with Exceptional Structure Groups. PhD thesis, University of Edinburgh, 2011.

[11] Dietmar A Salamon and Thomas Walpuski. Notes on the octonions. In Proceedings of the Gökova Geometry-Topology Conference 2016, 2017. https://arxiv.org/abs/1005.2820.

[12] Simon Salamon. Riemannian geometry and holonomy groups, volume 201 of Pitman Research Notes in Mathematics Series. Longman Scientific \& Technical, Harlow; copublished in the United States with John Wiley \& Sons, Inc., New York, 1989.

[13] Andrew Strominger, Shing-Tung Yau, and Eric Zaslow. Mirror symmetry is T-duality. Nuclear Physics B, 479(1):243 - 259, 1996.

Université DU QuÉBEC À MontrÉAL

DÉPARTEMENT DE MATHÉMATIQUES

Case postale 8888, succursale centre-ville, Montréal (Québec), H3C 3P8, Canada

E-mail address: gavin.cf.ball@gmail.com

MCMASTER UNIVERSITY

Department of Mathematics \& Statistics

Hamilton, ON, Canada, L8S 4K1

E-mail address: madnickj@mcmaster.ca 